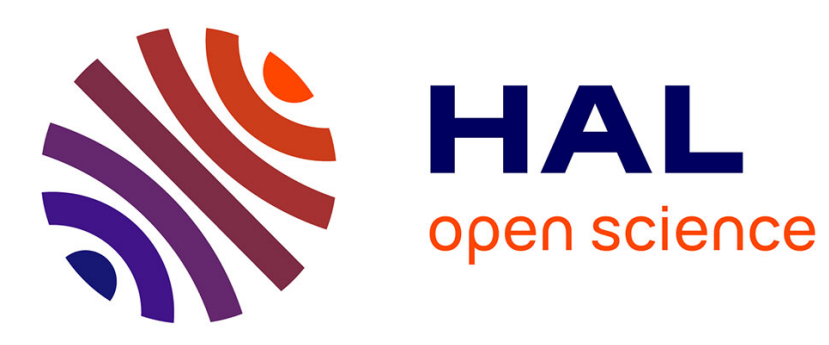

\title{
Modulated equations of Hamiltonian PDEs and dispersive shocks
}

Sylvie Benzoni-Gavage, Colin Mietka, L. Miguel Rodrigues

\section{To cite this version:}

Sylvie Benzoni-Gavage, Colin Mietka, L. Miguel Rodrigues. Modulated equations of Hamiltonian PDEs and dispersive shocks. Nonlinearity, 2021, 34 (1), pp.578-641. 10.1088/1361-6544/abcb0a . hal-02365963v1

\section{HAL Id: hal-02365963 https://hal.science/hal-02365963v1}

Submitted on 22 Nov 2019 (v1), last revised 10 Feb 2021 (v2)

HAL is a multi-disciplinary open access archive for the deposit and dissemination of scientific research documents, whether they are published or not. The documents may come from teaching and research institutions in France or abroad, or from public or private research centers.
L'archive ouverte pluridisciplinaire HAL, est destinée au dépôt et à la diffusion de documents scientifiques de niveau recherche, publiés ou non, émanant des établissements d'enseignement et de recherche français ou étrangers, des laboratoires publics ou privés. 


\title{
M odulated equations of Hamiltonian PDEs and dispersive shocks
}

\author{
Sylvie Benzoni-Gavage Colin M ietka ${ }^{\mathrm{y}} \quad$ L. Miguel Rodrigues ${ }^{\mathrm{z}}$
}

November 22, 2019

\begin{abstract}
A bstract
M otivated by the ongoing study of dispersive shock waves in non integrable systems, we propose and analyze a set of wave parameters for periodic waves of a large class of Hamiltonian partial dierential systems | including the generalized K orteweg \{de Vries equations and the Euler\{Korteweg systems | that are wellbehaved in both the small amplitude and small wavelength limits. We use this parametrization to determine ne asymptotic properties of the associated modulation systems, including detailed descriptions of eigenmodes. As a consequence, in the solitary wave limit we prove that modulational instability is decided by the sign of the second derivative / with respect to speed, xing the endstate / of the B oussinesq moment of instability; and, in the harmonic limit, we identify an explicit modulational instability index, of Benjamin\{Feir type.
\end{abstract}

Keywords: Whitham modulated equations, periodic traveling waves, Hamiltonian dynamics, harmonic limit, soliton asymptotics, dispersive shock, modulational instability, abbreviated action integral, general ized K orteweg \{de Vries equations, Euler $\{\mathrm{K}$ orteweg systems.

A M S Subject Classications: $\quad$ 35B 10; 35B 35; 35Q 35; 35Q51; 35Q 53; 37K 05; 37K 45.

\section{Contents}

1 Introduction 2

This work has been supported by the French National Research A gency projects NABUCO, grant ANR-17-CE40-0025, B OND, grant ANR-13-BS01-0009-01, and by the LABEX MILY ON (ANR-10-LABX0070) of Universid de Lyon, within the program ' Investissements d'A venir ' (ANR-11-IDEX-0007) operated by the French National Research A gency (ANR).

${ }^{\mathrm{Y}}$ The doctoral scholarship of CM was directly supported by A NR-13-B S01-0009-01.

${ }^{z}$ Research of LMR has received funding from the ANR project BoND (ANR-13-BS01-0009-01) and the city of Rennes. 
2 Various formulations of modulated equations 13

2.1 General framework . . . . . . . . . . . . . . . . . . . . 13

2.2 Modulated equations in terms of constants of integration . . . . . . . 14

2.3 An important averaged variable . . . . . . . . . . . . . . . 16

2.4 Alternative form of modulated equations . . . . . . . . . . . . . . . 18

3 A symptotic expansions of parameters 24

3.1 Expansions of action derivatives $\ldots \ldots \ldots \ldots$

3.2 Expansions of modulated variables $\ldots \ldots \ldots$

3.3 Extending the parametrization . . . . . . . . . . . . . . 33

4 A symptotics of the modulation system 36

4.1 Extending the averaged Hamiltonian $\ldots \ldots \ldots$

$4.2 \quad$ Basic features of the limiting modulated systems . . . . . . . . . . . . . . 37

4.3 Explicit formulas for the limiting modulation systems . . . . . . . . . . 41

5 A symptotics of the modulation eigenelds 45

5.1 Small amplitude regime. . . . . . . . . . . . . . . . . . . 45

5.2 Small wavenumber regime $\ldots \ldots \ldots \ldots . \ldots . \ldots . \ldots$

A ppendix

A Explicit formula for the modulational-instability index . . . . . . . . 50

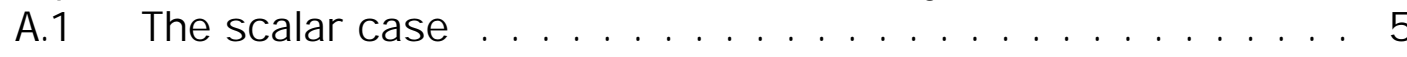

A.2 The system case $\ldots \ldots \ldots \ldots \ldots$

$\begin{array}{ll}\text { References } & 60\end{array}$

\section{Introduction}

M otivated by the study of dispersive shock waves, we investigate some of the universal properties of modulated equations, for a large class of Hamiltonian systems of partial dierential equations (PDE) that contains several models of mathematical physics and in particular generalized versions of the K orteweg $\{$ de $V$ ries $(K d V)$ equation and dispersive modications of the Euler equations for compressible uids - among which we nd the uid formulation via M adelung's transform of the nonlinear Schr odinger (NLS) equations. To place our results in context, we rst recall what are modulation systems, dispersive shock waves and their expected role in dispersive regularization. Large parts of this preliminary discussion are exploratory and conjectural since we are still lacking a rigorous analysis of dispersive shock waves and vanishing dispersive limits at the level of generality considered here. Indeed the present analysis is precisely designed as a rst step towards a general mathematically rigorous theory, still to come, and some elements of the preliminary discussion might be thought of as a roadmap for this ultimate goal. 


\section{Hamiltonian systems of Korteweg type}

As in [BGNR13, BGNR14, BGMR16, BGMRarl, we consider some abstract systems of the form

$$
@ U=@(B \quad H[U]):
$$

where the unknown $U$ takes values in $R^{N}$ with $N 2 f 1 ; 2 g, B$ is a symmetric and nonsingular matrix so that B @ is a skew-symmetric dierential operator, and $\mathrm{H}[\mathrm{U}]$ denotes the variational derivative at $\mathrm{U}$ of $\mathrm{H}=\mathrm{H}\left(\mathrm{U} ; \mathrm{U}_{\mathrm{x}}\right)$. We specialize to classes of systems satisfying more precise conditions described in A ssumption 1, Shuciently large to include quasilinear versions of the Korteweg\{de Vries equation and the abovementioned Euler \{ Korteweg systems, hence also hydrodynamic formulations of the nonlinear Schr odinger equations. In the former and henceforth spatial derivatives are denoted either as @ or as $x$.

A ssociated with the invariance of $\mathrm{H}$ by time and spatial translations comes the fact that smooth solutions of (1) also satisfy the local conservations laws

$$
\begin{aligned}
& @\left(H\left(U ; U_{x}\right)\right)=@ \frac{1}{2} H[U] B H[U]+r_{U_{x}} H\left(U ; U_{x}\right) @(B \quad H[U]) \\
& @(Q(U))=@\left(Q[U] B H[U]+r_{U_{x}} H\left(U ; U_{x}\right) @(B \quad Q[U]) H\left(U ; U_{x}\right)\right)
\end{aligned}
$$

for the Hamiltonian density $\mathrm{H}$, generating the time evolution, and the impulse $\mathrm{Q}$, given by $Q(U):=\frac{1}{2} U \quad B{ }^{1} U$, generating spatial translations. See Section 2 for details.

\section{Modulated equations}

Modulated equations are expected to govern the evolution of modulated periodic wavetrains (also called weakly deformed soliton lattices by Dubrovin and Novikov [D N89, [DN93|). Starting from a system of PDEs, such as (1), , admitting families of periodic traveling waves, one may derive modulation equations through an averaging procedure, which yields PDEs on large space-time scales for the local parameters of the waves. The corresponding ansatz, expected to approximate solutions to the original PDEs, looks like one periodic wave train on small scales but have variable wave parameters on larger scales, hence exhibit varying amplitude and wavelength on these large scales.

A robust way to obtain them is to consider a two-scale formal asymptotic expansion combining slow arbitrary variables and single-phase fast oscillations,

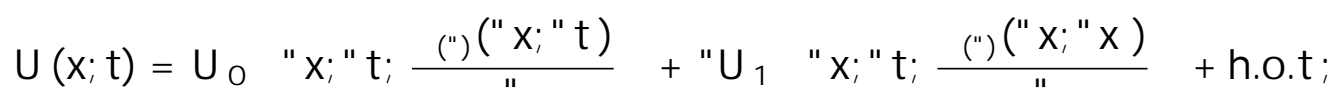

with $0<" \quad 1$,

$$
\begin{aligned}
& { }_{(")}(X ; T)={ }_{0}(X ; T)+{ }{ }_{1}(X ; T)+\text { h.o.t; } \\
& U_{j}(X ; T ;) \text { one-periodic in } ; \quad j=0 ; 1 ;
\end{aligned}
$$


where $\mathrm{X}=$ " $\mathrm{x}$ and $\mathrm{T}=$ "t denote some rescaled spatial and time variables respectively. A leading-order identication shows that for all $(X ; T)$,

$$
\underline{U}():=U_{0}(X ; T ; k(X ; T))
$$

must be the prole of a periodic, traveling wave solution to the original system, here $(1)$, of (spatial) period $(X ; T)=1=k(X ; T)$ and speed $c(X ; T)=!(X ; T)=k(X ; T)$, with $\mathrm{k}=$ @ 0 and ! = @ 0 . This al ready leaves as a constraint @ $\mathrm{k}=$ @ !, an equation known as the conservation of waves equation.

The missing part of the time evolution is obtained from constraints for the resolution of the next step of the identication. Indeed this step yields an ane equation for $U_{1}$, with

linear part essentially 17 given by the linearization about $\underline{U}$ of the original system, in the frame moving with speed $c$, acting on functions of with the same period as $\underline{U}$; source terms depending only on $U_{0}, 0$ and 1 .

The possibility to solve this step is then equivalent to the orthogonality (for the $\mathrm{L}^{2}$-scalar product in the -variable) of source terms to the kernel of the adjoint of the linear operator, a constraint automatically satised by the $\quad 1$-part of source terms. N ow it turns out that elements of the latter kernel are in correspondence with local conservations laws for the original systems. In the present case the conservative nature of (1), (R), (B) is directly linked to the presence in the kernel of the adjoint of the linearization of respectively constant functions, $\mathrm{Q}[\underline{\mathrm{U}}]$ and $\mathrm{H}[\underline{\mathrm{U}}]$. Orthogonality to those then yields time evolution equations for the averages of the quantities involved in local conservation laws. Note however that for traveling waves such as $\underline{U}, H[\underline{U}]$ is already a linear combination of $\mathrm{Q}[\underline{\mathrm{U}}]$ and constants so that the local conservation law for the averaged of $\mathrm{H}$ does not generate a new independent equation but an entropy for the modulated system.

The upshot of the detailed process (for which we refer to [NR13, B GNR14I) $s$ the modulated system

@ $k$ @ ! $=0$

(5c) @ $\mathrm{q} h Q(\underline{U}) i$

$$
\text { @ } h \underline{U} i \text { Q }(h B \quad H[\underline{U}] i)=0
$$

where brackets hi stand for mean values over the period ( $X ; T)$. A few comments on dimensions are worth stating (and we refer to [RDd13] for a more detailed discussion). The original Hamiltonian system (1 [ counts $\mathrm{N}$ equations whereas (5a) $(5 \mathrm{~b})-(5 \mathrm{C})$ involves $\mathrm{N}+2$ equations. From the way the modulated system has been derived it should be clear that this $\mathrm{N}+2$ breaks into 1 for the number of wavenumber variables 2 and $\mathrm{N}+1$

\footnotetext{
${ }^{1} U p$ to a rescaling from to , normalizing period to 1 .

${ }^{2}$ The nonlinear Schr odinger equations (in original formulations) form typically a case with a twodimensional group of symmetries. Their reduction to hydrodynamic form lowers the symmetry dimension by 1 but adds a conservation law.
} 
for the dimension of the span of $F[U]$, with $F$ ranging along functionals that satisfy a conservation law along the ow of $(1) \square \underline{U}$ being a periodic traveling wave. In the identication process, the number of independent averaged conservation laws, $\quad \mathrm{N}+1$ here, arises as related to the dimension of the kernel of the adjoint of the linearization in a moving frame, restricted to functions of the same period. This dimension must also be the dimension of the kernel of the adjoint of the linearized operator itself, hence the dimension of the family of periodic waves with xed period and speed (associated with the abovementioned wavenumber). Thus the formal argument also carries the fact that the dimension of the modulated system ( $\mathrm{N}+2$ here) diers from the dimension of the family of periodic traveling waves ( $N+3$ here) by the number of wavenumbers ( 1 here), hence agrees with the dimension of periodic wave proles identied when coinciding up to translation (again $\mathrm{N}+2$ ). The missing piece of information, about phase shifts, may be recovered a posteriori by solving @ $0=1, @_{0}=k$. As proved for instance in [BGNR 14 A ppendix B], to a large extent, the present informal discussion on dimensions may be turned into sound mathematical arguments.

As already pointed out in Whitham's seminal work [Whig9] for K dV and NLS, it is possible to use the variational structure of systems such as (1) to derive (5a). $(5 \mathrm{~b}) \cdot-(5 \mathrm{c})$ ) from least action considerations, instead of the geometrical optics expansion (4). For recent accounts of this variational derivation the reader is referred to [K an00, B ri 17]. As for the class of systems considered here, the corresponding form in terms of an action integral along periodic wave proles was explicited in [B(GNR13] and subsequently crucially used in [BGMR16] BGMRar],

A simple situation where one expects that the dynamics of (1) is well-described by a slow modulation ansatz similar to (4), hence obeys at leading-order a suitable version of $(5 a)-(\overline{5 b})-(\overline{5 c})$ is in the large-time regime starting from a smooth and localized perturbation of a single periodic traveling wave of (1), which should correspond to a nearly constant solution to (5a). (5b). (5c). Y Yet, though it is arguably the simplest relevant regime, a rigorous validation of the foregoing scenario has been obtained for none of the equations considered here. See however [BGNR14] for a spectral validation on the full class (1) $\square$ [Rod18] for a linear validation on KdV, and [J NRZ13, ]NRZ14] for full validations but on parabolic systems.

\section{Small dispersion limit}

Our present contribution is rather focused in regimes involving solutions to (5a) $(5 b),-(5 c)$ covering the full range of possible amplitudes, and known as dispersive shock waves. These are typically expected to play a key role in the regularization of shocks by weak dispersion.

This regime may be described by introducing a small wavenumber parameter " > 0 and moving to rescaled variables $(X ; T)=(" \mathrm{X} ; " \mathrm{t})$. Looking rst for a non oscillatory slow expansion (instead of (4))

$$
U(x ; t)=U_{0}(" x ; " t)+" U_{1}(" x ; " t)+\text { h.o.t ; }
$$


suggests that $U_{0}$ should satisfy

@ $\mathrm{U}_{0}=@\left(B r u H\left(U_{0} ; 0\right)\right)$;

a rst-order system of conservation laws. To make the discussion more concrete, let us temporarily focus on the $\mathrm{KdV}$ case, where (1) Decomes in slow variables

$$
\text { @ } v+\text { @ }\left(\frac{1}{2} \mathrm{v}^{2}\right)+{ }^{2} \mathrm{Q}^{3} \mathrm{v}=0
$$

and (7) reduces to the Hopf | or inviscid Burgers | equation

$$
\text { @ } \mathrm{v}+\text { @ }\left(\frac{1}{2} \mathrm{v}^{2}\right)=0 \text { : }
$$

In the $\mathrm{KdV}$ case, for nonnegative initial data and as long as the Hopf equation does not develop a shock, Lax and Levermore proved in [L[L83] that as " ! 0 the solutions to the above scaled $\mathrm{KdV}$ equation starting with the same initial datum converge strongly in $L^{2}$ to the solution of the Hopf equation. They also proved that after the shock formation a weak limit still exists but it does not solve the Hopf equation almost everywhere anymore. Instead there coexist some zones where the weak limit of $\mathrm{v}^{2}$ coincide with the square of the limit of $v$ and the latter solves the Hopf equation and other zones where this fails and the weak limit of $v$ does not satisfy an uncoupled scalar PDE but is the component hvi of a solution to the system of three equations given by $(5 a)-(5 b)-(5 c)$, specialized to $K d V$.

Since the seminal [ [L 83] there has been a lot of attention devoted to weak dispersion limits and their link to modulated equations (including some multi-phase versions), but for the moment all mathematically complete analyses are restricted to the consideration of completely integrable PDEs, such as $\mathrm{KdV}$, the modied $\mathrm{K}$ orteweg $\{$ de Vries equation, the cubic Schr odinger equations, the Benjamin-Ono equation and equations in their hierarchies. We refer the interested reader to [Ven85b, Ven85a, Ven87, Ven90, Wrig3, DVZ97, [ILM 99, Gra02, Mil02, TVZ04, TVZ06, PT07, MX11, MX12, M14, ] en15, M W 16] for original papers and to [E]LM03, EH16, Mil16] for a detailed account of progresses made so far in this direction. Note however that, whatever the precise denition we use for completely integrable PDEs, these correspond to specic nonlinearities and in fact few models are completely integrable.

To unravel some of the reasons why the completely integrable case is signicantly simpler to analyze | but still tremendously involved! |, let us step back a little and draw some analogies with the vanishing viscosity limit. The natural parabolic counterpart to the $\mathrm{KdV}$ equation above is the viscous Burgers equation

$$
\text { @ } v+@\left(\frac{1}{2} v^{2}\right)=" Q_{x}^{2} v:
$$

In this case, by using the Hopf-Cole [Hop50, Col51]transform, precisely introduced for this purpose, it is relatively easy to analyze the limit " ! $0^{+}$and check that solutions to the Burgers equation converge to the weak solution of the Hopf equation given by the Lax-Oleik formula.

The convergence towards a weak solution of the inviscid equation and the characterization of the vanishing viscosity limits by an entropy criterion has been extended even 
beyond the scalar case [01e57] to solutions to systems starting from initial data with small total variation [BB 05]. At the heart of these general treatments lies an understanding not only of the limiting slow behavior encoded by the solution of the inviscid equation but also of the fast part essentially supported near discontinuities of the inviscid solution. To give a heuristic avor of the latter, let us focus again on the scalar case and zoom from ( $T ; X)$ to $(T ;:)=(T ;(X \quad(")(T))=")$, with 0() describing the position of a discontinuity of the limiting solution $V_{0}$ and $X$ living in a neighborhood of the latter discontinuity. Then the identication of powers of "suggests that the fast local structure, at time $T$ near the discontinuity located at $\quad 0(T)$ should be described by a front of the Burgers equation (in fast variables) traveling with velocity @ o $(T)$ (satisfying the Rankine-Hugoniot condition) and joining $v_{0}\left({ }_{0}(T) ; T\right)$ to $v_{0}\left({ }_{0}(T)^{+} ; T\right)$ (where denote limits from above or from below). The existence of such viscous fronts plays a deep role in both the heuristic and rigorous treatment of the vanishing viscosity limit. In particular, even the rigorous masterpiece by Bianchini and B ressan [BB 05] proceeds through such a local traveling-wave decomposition of solutions.

In the dispersive case the possibility of this global-slow/local-fast scenario fails al ready in general by the non existence of the required traveling fronts. Indeed, elementary considerations show that whereas in the scalar diusive case the set of pairs of values that may be joined by a nondegenerate front is an open subset of $R^{2}$ and the selection of the speed coincides with the one given by the Rankine-Hugoniot condition, in the scalar K dV -like case this set is a submanifold of dimension 1. At this stage it should be clear that the understanding of what are the possible fast structures replacing viscous fronts, also called viscous shock waves, to join both sides of a shock should already provide a good grasp on the weak dispersion limit.

\section{The Gurevich\{Pitaevskii problem}

Leaving aside the possibility that the fast part of solutions could be given by well-localized elementary blocks, steady in the frame moving with the speed of the shock they regularize and interacting with the slow part only through their limiting values at innity, one is naturally led to the consideration as elementary fast blocks of unsteady patterns as in (4), mixing slow and fast scales but whose limits at 1 are purely slow, that is, constant with respect to the fast variable. M odulated periodic wave trains may reach these limiting constant states in two ways

by letting their amplitude go to zero, they reach a constant state by asymptoting a harmonic periodic wavetrain oscillating about the reference constant;

by letting their wavelength go to zero, they converge to a solitary wave connected to the reference constant by its limiting trail.

From the foregoing considerations arises the question of determining when two given constant states may be joined by a relaxation wave of $(5 a)-(5 b) \sqrt{-(5 c)}$ in the sense that limiting values of the relaxation wave are parameters corresponding to either harmonic or 
solitary waves and the limiting values of the average part $\mathrm{hU} i$ ts the prescribed constants. The corresponding unsteady, oscillatory patterns, recovered through (4), arle referred to as dispersive shock waves. Note that the question diers from the investigation of classical relaxation waves of hyperbolic systems in at least two ways. On one hand, both harmonic wavetrains with a prescribed limiting value and solitary waves with a prescribed endstate form one-dimensional families (when identied up to translation) and the knowledge of through which harmonic train or solitary wave given constant states may be joined is an important part of the unknown elements to determine. On the other hand the modulated system (5a)-(5b)-(5c) is a priori not dened at the limiting values and yet the hope to match dispersive shock waves with solutions to (7) heavily relies on the expectation that in both limits | solitary or harmonic | (5b) uncouples from the rest of the system and converges to (7)).

It is worth stressing that even though one expects to obtain, afterwards, a multi-scale pattern through (4) the foregoing problem is "-free. It is a dispersive analogous to the determination of conditions under which two constants may be joined by a viscous front. Note that in the viscous version of the problem such considerations are then included in the denition of admissibility of weak solutions to (7), and expected to determine reachability by vanishing viscosity limits. Notably, in the classical Riemann problem, one considers how to solve (7) starting from an initial datum taking one value up to some point then another value, by gluing constants, relaxation waves, admissible shocks and contact discontinuities. Solutions to the Riemann problems may then be used themselves as elementary blocks to solve the general Cauchy problem for (7) [designed from vanishing viscosity considerations). See for instance [Ser99, B re00I for background on the classical Riemann problem. Likewise, in the dispersiveless limit, the Gurevich\{Pitaevskii problem consists in joining two given constants on two complementary half-lines with constant sectors, relaxation waves of (7) and relaxation waves of (5a)-(5b))-(5c) the junction between solutions to $(7)$ and solutions to $(5 a)-(5 b)-(5 c)$ being understood in the sense mentioned herein-above. This approach was introduced for $\mathrm{KdV}$ by Gurevich and Pitaevskii in [GP73] and has been referred to as the Gurevich \{Pitaevskii problem since then, or sometimes the dispersive Riemann problem.

It must be stressed that as for Riemann problems in the weak dissipation limit, the Gurevich \{Pitaevskii problems are expected to carry a wealth of information on the weak dispersion regime. We already pointed out that a fully rigorous treatment of the weak dispersion limit is for the moment restricted to some equations, associated with $L$ ax pairs including a scalar Schr odinger operator and completely integrable through inverse scattering transforms. Unfortunately the same is true for the associated Gurevich\{Pitaevskii problems. Indeed modulated systems of those particular systems inherit from the Lax pair representation of the original system, a family of strong Riemann invariants, given by edges of Lax spectral bands; see [DN74, [DMN76, FFMM 80, DN83, F[86, Pav87] for original papers pointing this connection. The latter observation was certainly the main motivation for the introduction and the study of the classes of hyperbolic systems possessing a complete set of strong Riemann invariants, a class coined as rich by Serre [Ser00, Chapter 12] and as semi-Hamiltonian by Tsarev [T \$a85, Tsa90, Tsa00l. Along a relax- 
ation wave of a rich system all but one Riemann invariant are constants. M oreover in both the solitary wave limit and the harmonic limit of PDEs associated with such Lax pairs one of the Lax spectral gaps closes so that the Riemann invariant varying along a relaxation wave of such a rich modulated system connecting two harmonic/solitary limits is actually merging in both limits with one of the steady Riemann invariants. This makes the relaxation wave problem considerably simpler to solve, at least as far as determining which states may be connected and what are the trail and edge speeds of the pattern.

Given its particular importance for some classes of applications, there have been a few attempts to propose solutions to the Gurevich\{Pitaevskii not relying on strong Riemann invariants. One of the most remarkable attempt is due to $\mathrm{El}$ and the reader is referred to [EH16] for details on the method and to [E[05, Hoe14] for two instances of application. The elegant method of El provides answers consistent with rigorous analyses of integrable cases and displays reasonably good agreement with numerical experiments. Y et unfortunately, so far, it still lacks strong theoretical support, even of a formal heuristic nature. Elucidating the mathematical validity of the approach of EI may be considered as one of the key problems of the eld.

To conclude the exploratory part of the paper, we point out that even from a formal point of view there are at least two important features of the weak dispersion limit that we have left aside and on which we comment now.

Remark 1. System (5a)-( $5 \bar{b})$-(5c) is itself a | hopefully hyperbolic | rst-order system so that it may be expected to develop shocks in nite time and the expansion in (4) to suer from a nite-time validity (in the slow variables) in the same way as the relevance of (6) stops when the corresponding solution to (7) forms a shock. Yet the formal process itself hints at an "-dispersive correction to (5a)-(5[b)-(5E)] see for instance [Rod 18] !. so that the phenomena may be thought itself as a weak dispersion limit in the presence of wave-breaking at the level of (single-phase) modulation equations, suggesting the presence of oscillations at this level, and resulting in a two-phase oscillation pattern at the original level. For K dV a compatible scenario (with arbitrary number of phases) was proposed in [FFM 80] within the terminology of integrability by inverse scattering; it was subsequently recast in terms of averaged modulation equations in [EKV01] and proved to hold in [GT02 Gra04]. Note that the prediction includes a description of where 0-phase, 1-phase and 2-phases patterns live in the space-time diagram.

Remark 2. There has been considerable eort devoted to the description of what is seen on a zoom in a neighborhood of a wave-breaking point. This results in a dierent asymptotic regime and a suitable scenario was rst proposed by Dubrovin [Dub06] and then proved for various integrable PDEs in [CG09, CG12, BT13].

\section{Structure of general modulated systems}

As far as the formal description of dispersive shocks by means of modulated equations is concerned, multi-scale regions are connected to single-scale ones by either one of the two asymptotic regimes corresponding to the small amplitude limit - when the amplitude 
of the waves goes to zero - and the solitary wave limit - when the wavelength of the waves goes to innity. The understanding of both these regimes is a crucial step towards the actual construction of dispersive shocks. In particular, to analyze rarefaction waves of modulated equations connecting such asymptotic regimes, one needs to elucidate the hyperbolic nature of its eigenelds in both distinguished limits. Indeed, as for the classical Riemann problem, the resolution of the Gurevich \{Pitaevskii problem crucially relies on the hyperbolicity and the structure of the eigenelds of modulated equations. This requires a study not only of averaged quantities involved in the conservative formulation but also of their derivatives, as appearing in the expanded, quasilinear form.

$W$ ith this respect, note that unfortunately, the formulation of modulated equations in terms of what is arguably the most natural set of wave parameters blows up in the solitary wave limit. This issue has been partially resolved by $\mathrm{EI}$ [EI05] by replacing one of the parameters with the so-called conjugate wave number. However, this new parametrization is in general limited to the large wavelength regime.

One of our main contributions here is to provide a global set of parameters. For the latter, we prove in great generality that

it may serve as a parametrization of periodic wave proles (identied up to spatial translation) exactly when the original averaged quantities ( $k ; h \underline{U} i ; h Q(\underline{U}) i)$ can, that is, as proved in [BGNR 14, A ppendix B] and [BGMR16, Theorem 1], exactly when wave proles with xed period form an $(N+1)$-dimensional manifold (when identied up to spatial translation);

in these variables the modulated system possesses an Hamiltonian formulation, with Hamiltonian function the original averaged Hamiltonian energy (see Theorem 1);

these variables may be extended to solitary-wave and harmonic limits in such a way that the modulated system admits regular limits even in quasilinear form (see Theorem 4).

The proposed system of coordinates already appeared for the Euler $\{$ K orteweg system in mass Lagrangian coordinates in [CS95] (also see [BG13] for an account of Gavrilyuk Serre's result with our notation), but its signicance remained unclear. As we show hereafter, it turns out to apply to our more general framework, and to give new insight on modulated systems. The point is to replace the conserved variable $h Q(\underline{U}) i$ in Equations (5a)-(5b)-(5c) by another one, denoted merely by hereafter. This new variable tends to zero when the amplitude of the wave tends to zero | as the amplitude squared, as we shall see later on |, and has a nite limit when $k$ goes to zero, that is, in the solitary-wave limit. Remarkably enough, this quantity can be dened as simply as

$$
:=\frac{1}{\mathrm{k}}(\mathrm{hQ}(\underline{\mathrm{U}}) \mathrm{i} \mathrm{Q} \quad(\underline{\mathrm{hU}} \mathrm{i})):
$$

It turns out that, as far as smooth solutions are concerned, the modulation equations take 
the alternative form

$$
\text { @ @ }{ }_{M}^{0} A=B @\left(r_{k i ;} M H\right) \text {; }
$$

where

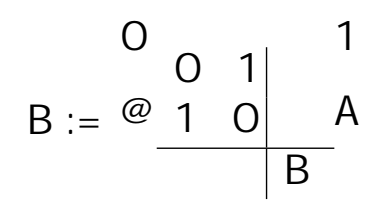

is symmetric and nonsingular and

$$
M:=h \underline{U} \underline{i} ; \quad H:=h H[\underline{U}] i \text { : }
$$

The Hamiltonian structure of System (9) provides a form of symmetry in the spirit of Godunov's theory of hyperbolic systems. Nevertheless, this form does not automatically provide energy estimates nor imply hyperbolicity because, as our expansions show (see Remark 13), the associated potential, natural symmetrizer is not denite in either one of the limits.

System (9) has also an appealing symmetric form with respect to the distinguished limits, $k$ ! 0 corresponding to the long wavelength limit and ! 0 to the small amplitude limit. $Y$ et another upshot of our analysis is a strong, somewhat surprising asymmetry as regards the asymptotic nature of the eigenelds. In the solitary wave regime, the hyperbolicity of the modulated equations is equival ent to its weak hyperbolicity and may persist even at the limit in the presence of the solitary wave speed as a double root. We see this striking property as a consequence of the strong separation of scales displayed in asymptotic expansions of large wavelength proles (see Remark 16) Bly contrast, in the harmonic wave regime, in general the hyperbolicity of modulated equations is lost at the limit, the characteristic speed corresponding to the group velocity being non semi-simple | associated with a Jordan block of height two. See Theorems 5 and 6.

We stress that many asymptotic properties of the modulation systems are much easier to study once a limiting system has been identied. This is precisely where we benet from our new set of parameters. In particular, once Theorems 1 and 4 are known it is relatively easy to derive the most basic properties of the modulation systems, both mentioned and used in the preceding formal discussion of dispersive shocks. For instance Corollary 5 contains that at both limits the modulation system split into a block coinciding with the original dispersionless system, System (7), and a $2 \quad 2$ block with double characteristic given by either the solitary wave speed at the long wavelength limit or with the harmonic linear group velocity at the small amplitude limit. Y et, even with good variables in hands, some further properties require ner details of higher-order expansions.

Our new set of parameters enables us to show how the eigenelds of modulated equations degenerate in the small amplitude and the large wavelength limits (see Theorems 7 and B) but it relies on a more involve analysis. The main upshots are that 
Near the harmonic limit, we derive explicit conditions determining modulational instability (see Theorem 7 and A ppendix A), those being known in some cases as the Benjamin \{Feir criteria.

Near the soliton limit, we prove that modulational instability is determined by exactly the same condition ruling stability of solitary waves and, as proved in [BGMRar], co-periodic stability of nearby periodic waves, that is, it is decided by the sign of the second derivative / with respect to speed, xing the endstate / of the Boussinesq moment of instability.

For the conclusions mentioned here it should be emphasized that it is relatively easy to support wrong deceptive claims when arguing formally; see for instance Remark 15. Another somewhat surprising, but not unrelated (see Remark 16), discrepancy between both limits is that the convergence of eigenvalues splitting from the double root is exponentially fast in the solitary limit.

We insist on the fact that, surprising or not, the properties of the modulation systems discussed in the present contribution are proved here for the rst time for a class of systems not restricted to integrable systems.

We conclude this introductory section with a few words on the nature of proofs contained in the rest of the paper. The most elementary ones are purely algebraic manipulations. For the other ones we rely on asymptotic expansions of the abbreviated action integral of the prole $O D E$, and of its derivatives up to second order. These were derived in detail in [BGMRarl]and used there to deduce some consequences on the stability of periodic waves with respect to co-periodic perturbations. As we show in the present paper, that asymptotic behavior gives insight on the modulated equations as well.

The general setting and various formulations of modulated equations are presented in Section 2. A symptotic properties of the alternate parametrization are established in Section 3. Limits of the modulated system are derived in Section 4. Figenelds are studied in Section 5. A ppendix A Gontains explicit modulational instability criteria for the harmonic limit.

A cknowledgement. The rst and third author would like to express their gratitude to Gennady El, Sergey Gavrilyuk, Mark Hoefer and Michael Schearer for enlightening discussions during the preparation of the present paper.

$M$ atrix notation. A long the text, in matrices, 0 may denote scalar, vector or matrixvalued zeroes. Moreover empty entries denote zeroes and entries denote values that are irrelevant for the discussion and may vary from line to line. 


\section{Various formulations of modulated equations}

\subsection{General framework}

As announced in the introduction, we consider abstract systems of the form

$$
@ U=@(B \quad H[U]):
$$

where the unknown $U$ takes values in $R^{N}, B$ is a symmetric and nonsingular matrix, and $\mathrm{H}\left[\mathrm{U}\right.$ ] denotes the variational derivative at $\mathrm{U}$ of $\mathrm{H}=\mathrm{H}\left(\mathrm{U} ; \mathrm{U}_{\mathrm{x}}\right)$. For the sake of clarity, here, we shall mostly stick to A ssumption 1 Delow, all the more so when we are to apply results from [BGMRar]

A ssumption 1. There are smooth functions $f$, and with and taking only positive values, and a nonzero real number $b$ such that

$$
\begin{aligned}
& \text { either } N=1, U=v, H=e\left(v ; v_{x}\right) \text {, and } B=b \text {, } \\
& \text { or } N=2, U=(v ; u)^{\top} \text {, } \\
& \qquad H=\frac{1}{2}(v) u^{2}+e\left(v ; v_{x}\right) ; \quad B=\begin{array}{ll}
0 & b \\
b & 0
\end{array}
\end{aligned}
$$

with

$$
e\left(v ; v_{x}\right)=\frac{1}{2}(v) v_{x}^{2}+f(v)
$$

in both cases.

By denition we have

in the case $N=1, \quad H[U]=e[v]:=@ e\left(v ; v_{x}\right) @\left(@_{x} e\left(v ; v_{x}\right)\right)$,

in the case $\mathrm{N}=2$,

$$
H[U]=\frac{\frac{1}{2} q(v) u^{2}+e[v]}{(v) u} \quad:
$$

The impulse

$$
Q(U):=\frac{1}{2} U \quad B{ }^{1} U \text {; }
$$

generates $\mathrm{x}$-translations in that

$$
@ U=@(B \quad Q[U]):
$$

From the invariance of $\mathrm{H}\left(U ; U_{x}\right)$ with respect to $x$-translations, that reads in dierentiated form

$$
@_{(}\left(H\left(U ; U_{x}\right)\right)=H[U] @_{x} U+@_{x}\left(U_{x} r U_{x} H(U ; U x)\right) \text {; }
$$


stems the local conservation law

$$
@ Q(U)=@(U \quad H[U]+L H[U]) ;
$$

along smooth solutions of $(\sqrt{0})$ where

$$
L H[U]:=U_{x} r U_{x} H\left(U ; U_{x}\right) H \quad\left(U ; U_{x}\right)=v_{x} @_{x} e\left(v ; v_{x}\right) H \quad\left(U ; U_{x}\right):
$$

The modulated system (5a)-(5b)-(5ф) is also written as

@ $\mathrm{k}+$ @ ! =0;

@ $h \underline{U} \mathrm{i} @ \mathrm{hB} H[\underline{U}] \mathrm{i}=0$;

@ $h Q(\underline{U}) i @ \underline{h} \quad H[\underline{U}]+L H[\underline{U}] i=0$;

where $7 ! \underline{U}(X ; T ; \quad)$ is the prole of a periodic, traveling wave solution to (10) of (spatial) period $(X ; T)=1=k(X ; T)$ and speed $c(X ; T)=!(X ; T)=k(X ; T)$, and brackets hi stand for mean values over the period $(X ; T)$. A gain we refer to [BGNR14] for a formal derivation of the system from a geometrical optics expansion.

As proved in [BGNR14, A ppendix B] and [BGMR16. Theorem 1], the fact that System (5a)-( $5 b)$ - $(5 c)$ is a closed system, of evolution type, for initial data under consideration, is equivalent to the fact that, for each xed period, periodic wave proles under consideration form a non-degenerate manifold of dimension $\mathrm{N}+1$ when identied up to translation. In this case wave proles may be smoothly parametrized by ( $k$; $\underline{\text { U }} \mathrm{i} ; \mathrm{hQ}(\underline{\mathrm{U}}) \mathrm{i})$. As mentioned in the introduction, from the point of view of modulation theory, the range of validity of the latter parametrization is optimal. Yet these coordinates come with at least three serious drawbacks:

they are not very explicit so that within this set of coordinates the modulation system is hard to manipulate;

they are degenerate in the solitary-wave limit, losing two dimensions instead of one dimension;

they do not provide a clear variational form for the modulation system.

We rst recall how the rst and third issues may be xed by choosing a parametrization involving constants of integration of the wave prole ODEs.

\subsection{M odulated equations in terms of constants of integration}

For a traveling wave $U=\underline{U}(x \quad c t)$ of speed $c$ to be solution to $(\sqrt{0})$, there must exist $a$ $2 R^{N}$ such that

$$
(H+C Q)[\underline{U}]+=0 \text {; }
$$


which can be viewed as the Euler\{Lagrange equation $L[U]=0$ associated with the Lagrangian

$$
\mathrm{L}=\mathrm{L}\left(\mathrm{U} ; \mathrm{U}_{\mathrm{x}} ; \mathrm{C} ; \quad\right):=\mathrm{H}\left(\mathrm{U} ; \mathrm{U}_{\mathrm{x}}\right)+\mathrm{CQ}(\mathrm{U})+\mathrm{U}:
$$

Exactly as (B) was derived from (1) and the invariance by translation of $H\left(U ; U_{x}\right)$, it stems from the translational invariance of $\mathrm{L}\left(\mathrm{U} ; \mathrm{U}_{\mathrm{x}}\right)$ that solutions to $(15)$ are such that for some $2 \mathrm{R}$

$$
\operatorname{LL}[\underline{U}]=\text {; }
$$

where

$$
\text { LL [U ] : = } U_{x} \quad r \quad U_{x} L\left(U ; U_{x}\right) L \quad\left(U ; U_{x}\right):
$$

This more concrete point of view introduces as natural wave parameters their speed $C$ and the integration constants $2 \mathrm{R}^{\mathrm{N}}$ and $2 \mathrm{R}$. As discussed with more details in [BGNR13] BGMR16] | see in particular the proof of [BGMR16. Theorem 1] |, once these parameters are xed, non-constant periodic wave proles form a discrete set and the corresponding proles perturb smoothly with respect to parameters. Henceforth we will often omit to specify that one of the branches of periodic waves have been chosen. By doing so, we obtain (a discrete number of) natural parametrizations of wave proles.

As already pointed out in [EGNR13, BGMR16, BGMRarl, many key properties of periodic traveling waves are more neatly stated in terms of the wave-speed and constants of integration by introducing the abbreviated action integral

$$
(; c ; \quad):==_{0}^{Z}(H[\underline{U}]+C Q(\underline{U})+\underline{U}+) d ;
$$

whose denition involves a periodic prole $\underline{U}$ of fundamental period corresponding to parameter values $(; c ; \quad)$. The action provides a nice closed form of the modulated equations in (2) $-(\sqrt{3)})-(14)$ and it encodes the duality between constants of integration and averaged quantities. Indeed, let us recall from [B GNR13) the following.

Proposition 1. Under assumption 1, 1 consider an open subset of $\mathrm{R}^{\mathrm{N}+2}$ and

$$
(; C ; \quad) 2 \quad 7 !(\underline{U} ;) \quad 2 L^{1}(R) \quad(0 ;+1)
$$

a smooth mapping ${ }^{3}$ such that for each value of the parameters $(; c ; \quad)$, the function $\underline{U}=$ $(\underline{v} ; \underline{u})$ is a smooth, non-constant periodic solution to $(15)-(\sqrt{16})$, and is the fundamental period of $\underline{\underline{U}}$.

Then the function dened in (17) is also smooth on , and such that

$$
@=; \quad @={ }_{0}^{R} Q(\underline{U}) d x ; \quad r={ }_{0}^{R} \underline{U} d x:
$$

Corollary 1. In the framework of Proposition 1,

\footnotetext{
${ }^{3}$ That is, we choose one branch of waves.
} 
1. the system in (12)-(13) - (14) equivalently reads, as far as smooth solutions are concerned,

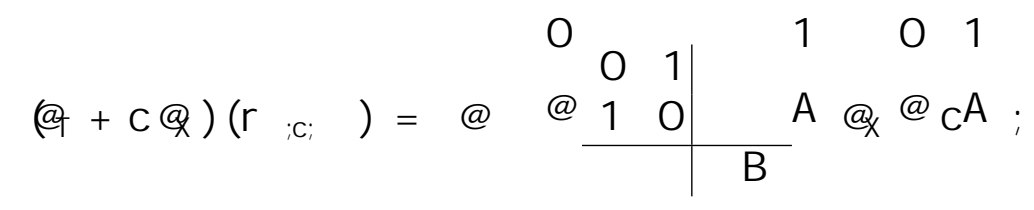

2. the mapping

$$
(; c ; \quad) 2 \quad 7 ! k=1=; \underline{h} \underline{i} i=\underline{1}_{0}^{R} \underline{U} d x ; h Q(\underline{U}) i=\underline{1}_{0}^{R} Q(\underline{U}) d x
$$

is a local dieomorphism if and only if

$$
\operatorname{det} r_{i c ;}^{2}(; c ; \quad) \in 0 ; 8(; c ; \quad) 2 \text { : }
$$

Remark 3. The simple, closed form in (9) of modulated equations is well-known for the $\mathrm{KdV}$ equation. It is for instance pointed out by Kamchatnov [K am00, eq. (3.135)], who says that 'despite the simple appearance of these equations, they are not very useful in practice'.

Exactly as pointed out in the introduction for System (9), the symmetric form of (19) does not readily imply that this system is hyperbolic. This would be the case if the matrix $\quad r_{i c ;}^{2} \quad$ were denite, which is not the case in general. As was shown in [BGMRar, Corollaries 1 and 2], in non-degenerate cases, $r_{i c ;}^{2}$ has a negative signature / or M orse index | equal to $\mathrm{N}$ for small amplitude waves and equal either to $\mathrm{N}$ or to $\mathrm{N}+1$ for those of large wavelength. In addition, as follows from [BGMR16], for $\mathrm{N}=1$ a denite Hessian matrix $r_{i ;}^{2}$ is incompatible with the spectral stability of the underlying periodic wave.

A $n$ important drawback of the formulation of modulated equations in the variables $(; \mathrm{C} ; \quad)$ is that all the quantities appearing in the time derivatives in (19) blow up in the solitary wave limit. Indeed, $@==1$ =k goes to innity when $\mathrm{k}$ goes to zero, as well as $r=h \underline{U} i$ and $@=h Q(\underline{U}) i$.

\subsection{An important averaged variable}

We claim that, despite their complicated and implicit form, Equations in (12)-(13)-(14) admit an equivalent form in a system of coordinates that is rather well suited for the study of dispersive shocks, in that it allows to take both the small amplitude limit and the solitary wave limit ( $k ! 0)$, in a most symmetric manner. We achieve this goal by replacing the conserved variable $\mathrm{hQ}(\underline{\mathrm{U}}) \mathrm{i}$ with another one, which we merely denote by , and that is given by

$$
:=\frac{1}{k}(h Q(\underline{U}) i Q \quad(\underline{h} \underline{i}))=\frac{1}{k} h Q(\underline{U} h \underline{U} i) i:
$$

As al ready pointed out above and proved below, this new variable tends to zero when the amplitude of $\underline{U}$ tends to zero and has a nite limit when $k$ goes to zero, that is when 
$\underline{U}$ eventually becomes a solitary wave prole. Indeed, rst we observe that goes indeed to zero when the amplitude of $\underline{U}$ goes to zero, because $k$ goes to the nonzero harmonic wave number and $\mathrm{hQ}(\underline{\mathrm{U}}) \mathrm{i}$ and $\mathrm{Q}(\underline{\mathrm{h}} \underline{\mathrm{i}})$ both go to the value of $\mathrm{Q}$ at the constant limiting state of the small amplitude wave. As to the limit when $k$ goes to zero, we see that

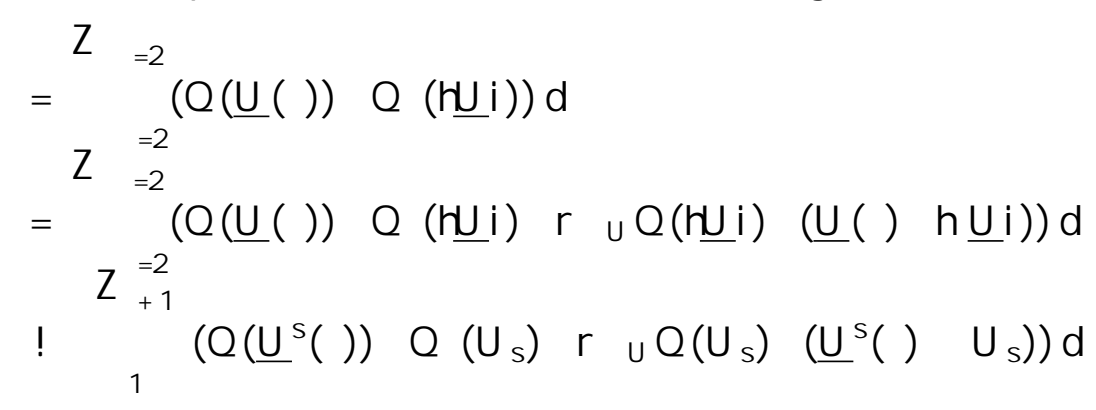

when goes to innity, where $\underline{U}^{s}$ denotes the limiting, solitary wave prole, homoclinic to $U_{s}$, the limit of $h U \mathrm{i}$. The asymptotic behavior of in these limits is proved in more details in Subsection 3.2.

A nother remarkable property of is that, at least for our main models of interest, scalar equations ( $\mathrm{N}=1$ ) and Euler $\{\mathrm{K}$ orteweg systems, one may determine the sign of in terms of parameters governing the traveling proles.

Proposition 2. Under Assumption 7

if $\mathrm{N}=1$ then has the sign of $\mathrm{b}$;

if $\mathrm{N}=2$ and $=\mathrm{Id}_{\text {, then }}$ has the sign of $\mathrm{b}_{2}$;

if $\mathrm{N}=2$ and 1 , then has the sign of $\mathrm{C}$.

Proof. The simplest case is for scalar equations, for which $Q(v)=v^{2}=(2 b)$, so that

$$
2 b=h \underline{v}^{2} i h \text { li } h \quad \underline{v i}^{2}>0
$$

by the Cauchy $\{S$ chwarz inequality, since $\underline{v}$ is not constant.

If $\mathrm{N}=2$ then $\mathrm{Q}(\mathrm{v} ; \mathrm{u})=\mathrm{v} u=b$. Yet, when 1 , it follows from (15) that $\underline{u}+(\mathrm{c} \underline{\mathrm{v}})=\mathrm{b}$ is constant so that the Cauchy $\{$ Schwarz inequality implies that

$$
h Q(\underline{v} ; \underline{u}) i Q \quad(h \underline{v} i ; h \underline{i})=\frac{C}{b^{2}}\left(\underline{h v}^{2} i h \quad l i h \quad \underline{v}{ }^{2}\right)
$$

is of the same sign as c. Indeed from the relation between $\underline{u}$ and $\underline{v}$ it follows that $\underline{v}$ is not constant since $\underline{U}$ is not constant. Likewise when $=I d, \underline{u}+{ }_{2}=\underline{v}$ is constant and

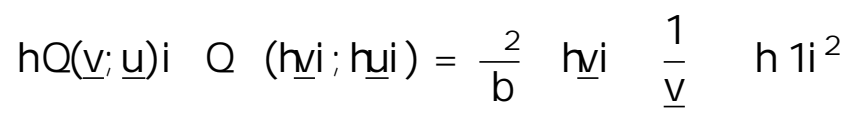

is of the sign of ${ }_{2}=b$ by the Cauchy $\{S$ chwarz inequality again, since, as $\underline{v}$ is non-constant, $P_{\underline{\nabla}}$ and $1 \stackrel{p}{\bar{v}}$ are independent. 
Remark 4. The case when $\mathrm{N}=2$ and $=$ Id includes Eulerian formulations of the Euler $\{$ K orteweg systems, whereas the case when $\mathrm{N}=2$ and 1 encompasses mass Lagrangian formulations of such systems. Each element of the latter class is conjugated to an element of the former and vice versa. As pointed out in [B ( $\mathrm{G} 13]$, correspondences respect traveling wave types, and, as proved in [BGNR14l, they also respect details of (the nonzero part of) the spectrum of linearizations about periodic waves. Obviously the foregoing proposition is consistent with corresponding conjugacies. Indeed, denoting with subscripts ${ }_{E}$ and $L$ quantities corresponding to each formulation, it follows from [B GNR14] that $b_{E}=1, b_{L}=1,(2)_{E}=C_{L}=b$ and

$$
\frac{\mathrm{E}}{\mathrm{K}_{\mathrm{E}}}=\frac{\mathrm{L}}{\mathrm{K}_{\mathrm{L}}}:
$$

\subsection{A Iternative form of modulated equations}

Returning to our general framework, we claim that variables ( $k ; ; M:=h \underline{U} i)$ may be used exactly when $(\mathrm{k} ; \mathrm{M} ; \mathrm{hQ}(\underline{\mathrm{U}}) \mathrm{i})$ may be used and that using the former yields an alternate formulation of the modulated equations (19) that still has a nice symmetric-looking structure, and is now well-suited for both the small amplitude limit $(\quad ! \quad 0)$ and the solitary wave limit $(k ! 0)$.

To begin with, note that the vector ( $k ; ; M)$ is deduced from $(k ; M ; h Q(\underline{U}) i)$ through the map ( $k ; M ; P) 7 !(k ;(P \quad Q \quad(M))=k ; M)$, which is obviously a (local) dieomorphism so that parametrizations are indeed equivalent. In particular, Corollary 1 proyides a characterization of when parametrization by $\left(k_{;} ; M\right)$ is possible.

Now we provide counterparts to Proposition 1 and Corollary 1 for variables ( $\left.k_{;} ; \quad M\right)$. Here, the role of in (19) is to some extent played by the averaged Hamiltonian

$$
\mathrm{H}:=\mathrm{hH}[\underline{\mathrm{U}}] \mathrm{i}:
$$

Remark 5. Remarkably the action integral and the averaged $\mathrm{Hamiltonian} \mathrm{H}$ are closely related. It follows indeed from the denition of in (17) and the expression of its derivatives in (8) that

$$
=\mathrm{H}+\mathrm{C} @+\mathrm{r}+@ \text { : }
$$

Would be strictly convex, we would recognize

$$
H=c @+r+@
$$

as being the conjugate function of .

Theorem 1. In the framework of Proposition 1, assume that the action dened in (17) has a nonsingular Hessian at all points in. Then the mapping $(; \mathrm{c} ; \quad) 7 !(\mathrm{k} ; ; \mathrm{M})$ dened by

$$
k=\frac{1}{;} ; \quad=\frac{1}{k}(h Q(\underline{U}) i Q \quad(h \underline{U} i)) ; \quad M=\underline{h U} i ;
$$

is a local dieomorphism. Moreover 
1. as a function of $(k ; ; M)$ the averaged Hamiltonian

$$
H:=h H[\underline{U}] \mathrm{i}
$$

is such that,

$$
@ H=C ; \quad @ H=k C ; \quad r_{M} H=h H[\underline{U}] i ;
$$

2. the modulated equations in (12)-(13)-(14) | or equivalently (19) | have a closed form in the variables $(k ;$; $M)$, which reads

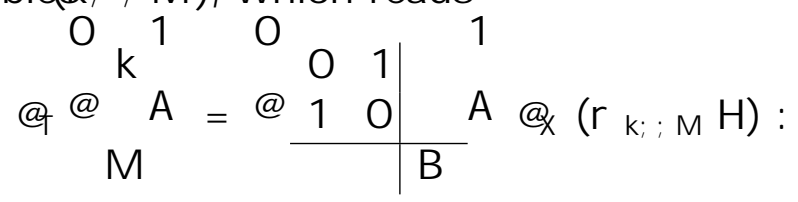

Proof. We have already established the rst assertion. However, for later use let us point out more precisely that the relations in (18) $\mid$ linked to the fact that is indeed an abbreviated action integral / imply that

$$
\begin{aligned}
& \mathrm{k}=\frac{1}{@} \text {; } \\
& M=\frac{r}{@} ; \\
& =@ \\
& @=\frac{1}{k} ; \quad \quad r=\frac{M}{k} ; \quad Q=+\frac{1}{k} Q(M):
\end{aligned}
$$

In order to compute the partial derivatives of $\mathrm{H}$ in the variables ( $\left.\mathrm{k}_{;} ; \quad M\right)$, it is expedient to use (20). Indeed, in this way, by combining classical cancellation of derivatives of conjugate functions with relations (24), we derive

$$
\begin{aligned}
@ H & =\frac{H}{k}+c \frac{Q(M)}{k}+\frac{M}{k}+\frac{1}{k}=c ; \\
@ H & =c k ; \\
r_{M} H & =c r Q(M)=h H[\underline{U}] \mathrm{i} ;
\end{aligned}
$$

where the last relation is obtained by averaging (15).

It follows at once that equations (12)-(13), which are also the rst and last lines of (19), are equally written as

$$
\begin{array}{ll}
@ \mathrm{k} & @(@ H)=0 ; \\
@ M & @\left(B r_{M} H\right)=0:
\end{array}
$$

So the only remaining task is to manipulate (19) to obtain an equation for. By using (23), (19) and the symmetry of $B$, one derives

$$
\begin{aligned}
(@+c @) & =(@+c @) @ \frac{Q(r)}{@} \\
& =@ @+r @+\frac{Q(r)}{@} @ c \\
& =@ @ \times c
\end{aligned}
$$


thus

$$
\text { @ = @ (@H): }
$$

Remark 6. The 'symmetric ' form of (22) readily implies that $\mathrm{H}$ is a mathematical entropy for this system. Indeed, along smooth solutions of (22) yre have

$$
@ H=@(@ H)(@ H)+\frac{1}{2}\left(r_{M} H\right) B r_{M} H
$$

by the symmetry of $B$. For the sake of consistency, we now check that this conservation law for $\mathrm{H}$ coincides with the averaging of (2) the original conservation law for $\mathrm{H} \mathrm{I}$ along wave proles. On one hand from (15), (16) and (20) stems

$$
\begin{aligned}
\operatorname{hr} \underline{U}_{x} H[\underline{U}] @_{(}(B H[\underline{U}]) i & =c h r \underline{U}_{x} H[\underline{U}] Q_{\underline{U}} \mathrm{i} \\
& =c(+c h Q(\underline{U}) i+M+H) \\
& =c k:
\end{aligned}
$$

On the other hand from (15) and the symmetry of $\quad B$ follows

$$
\begin{aligned}
& h \frac{1}{2} H[\underline{U}] \quad B \quad H[\underline{U}] i=\frac{1}{2} h H[\underline{U}] i \quad B h H[\underline{U}] i+c^{2} h Q(\underline{U} \quad M) i \\
& =\frac{1}{2} h H[\underline{U}] \mathrm{i} \quad \mathrm{BhH}[\underline{\mathrm{U}}] \mathrm{i}+\mathrm{c}^{2}(\mathrm{hQ}(\underline{\mathrm{U}}) \mathrm{i} \mathrm{Q} \quad(\mathrm{M})) \\
& =\frac{1}{2} h \mathrm{H}[\underline{\mathrm{U}}] \mathrm{i} \quad \mathrm{BhH}[\underline{\mathrm{U}}] \mathrm{i}+\mathrm{c}^{2} \mathrm{k} \text { : }
\end{aligned}
$$

Combining the foregoing with (21) proves the claim.

Remark 7. The conservation law (1]4) itself also admits a nice formulation in terms of $\mathrm{H}$. It equival ently reads

$$
\text { @ } h Q(\underline{U}) i=Q H \text {; }
$$

where

$$
\mathrm{H}:=\mathrm{k} @ \mathrm{H}+\mathrm{H}+\mathrm{M} \mathrm{r}_{\mathrm{M}} \mathrm{H} \quad \mathrm{H} \text {; }
$$

( $\mathrm{H}$ would be the conjugate function of $\mathrm{H}$ if this function were strictly convex). Indeed it al ready follows from previous computations that

$$
h L H[\underline{U}] i=k \quad H:
$$

M oreover from (15) and the symmetry of B we deduce

$$
\begin{aligned}
& \text { hU } \quad \mathrm{H}[\underline{U}] \mathrm{i}=\mathrm{M} \quad \mathrm{h} H[\underline{\mathrm{U}}] \mathrm{i} \quad 2 \mathrm{chQ}(\underline{\mathrm{U}} \quad \mathrm{M}) \mathrm{i} \\
& =M h H[\underline{U}] \mathrm{i} \quad 2 \mathrm{ck}
\end{aligned}
$$

so that the claim follows from (21). 
The quasilinear form of (22) reads

$$
@ @_{M}^{0} A=B r_{k ; m}^{2} H @ @_{M}^{1}{ }_{k}^{1} \text {; }
$$

where

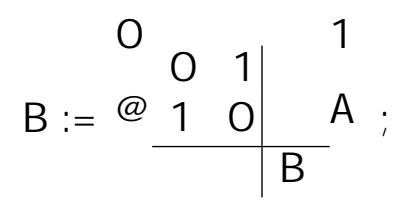

so that (22) is hyperbolic at points in the state space where the matrix $\quad \mathrm{Br}_{\mathrm{k} ; ;}^{2} \mathrm{M}$ is diagonalizable with real eigenvalues. A rst, natural approach to check hyperbolicity is to try and use the symmetry of the matrices $r_{k ; ;}^{2} \mathrm{M}$ and $\mathrm{B}$.

Corollary 2. In the framework of Theorem, if $\mathrm{H}$ is a strictly convex function of $(\mathrm{k} ;$; $\mathrm{M})$, then the modulated system (22) is hyperbolic.

Proof. This follows from the fact that the Hessian $\mathrm{r}_{\mathrm{k} ; i}^{2} \mathrm{M} H$ of $\mathrm{H}$ is a symmetrizer for (22) whenever $r_{k ; i}^{2} \mathrm{H}$ is positive denite. Indeed, as soon as $r_{\mathrm{k} ; i}^{2} \mathrm{M}$ is nonsingular the quasilinear form (28) of (22) is equivalent to

$$
r \underset{k ; i M}{2} H @{ }_{M}^{0} A=r \underset{k ; i}{2} H B r_{k ; i}^{2} H @{ }_{M}^{1} A \text { : }
$$

Since the matrix $\mathrm{r}_{\mathrm{k} ; ;}^{2} \mathrm{M} \mathrm{HBr} \underset{\mathrm{k}_{i ;}}{2} \mathrm{M}$ is symmetric, if in addition $\mathrm{r}_{\mathrm{k} ; ;}^{2} \mathrm{M}$ is positive denite then (22) is necessarily hyperbolic by a standard observation in the theory of hyperbolic systems (see for instance [Ser99, Theorem 3.1.6]).

However, our numerical experiments tend to show that $\quad r_{\mathrm{K}_{i ;}}^{2} \mathrm{M}$ is hardly ever denite positive [Mie17]. Moreover, as made explicit in Remark 13, our analysis proves that $\mathrm{r}_{\mathrm{k} ; i}^{2} \mathrm{M}$ is not denite positive in either one of the small amplitude limit and the large wavelength limit. Indeed, the upper diagonal block in the limits of $\mathrm{r}_{\mathrm{k} ; ;}^{2} \mathrm{M}$ found in Theorems 5 and $\$$ has signature $(1 ; 1)$, and therefore $\mathrm{r}_{\mathrm{k} ; i}^{2} \mathrm{M} H$ cannot be denite.

The main purpose of subsequent sections is to draw rigorous conclusions on the modulated system in quasilinear form (2B), in the small amplitude and soliton limits, when either ! 0 or $k$ ! 0 . Required expansions are derived from expansions of $r{ }_{i c ;}^{2}$ obtained in [BGMRar]. Thus, before going to the most technical part of the present paper, we need to point out the explicit connection between the Hessian of the averaged Hamiltonian $\mathrm{H}$ as as function of $(\mathrm{k} ; ; \mathrm{M})$ and the Hessian of the abbreviated action as a function of parameters $(; \mathrm{c} ;)$.

Proposition 3. In the framework of Theorem 1,

$$
r_{k ; ;}^{2} M^{2}=\frac{1}{k} A^{\top}\left(r_{; ;}^{2} ;{ }^{1} A \quad C B^{1} ;\right.
$$


with

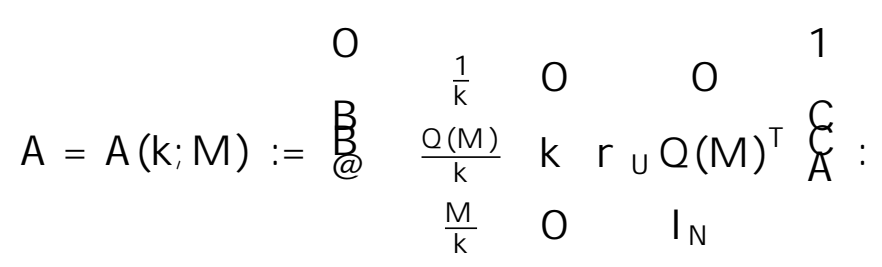

Proof. A long the proof we nd it convenient to use rst and second dierentials, denoted with $d$ and $d^{2}$, rather than gradients and Hessians. We proceed by dierentiating at points ( $k$; ; M ) (left implicit) in an arbitrary direction ( $k_{;} ;-M$ ) (made explicit). In the present proof all functions are implicitly considered as functions of $(\mathrm{k} ; \mathrm{M})$.

The starting point is the dierentiation of (20), alleady used in the proof of Theorem 1,

$$
01
$$

$$
d H\left(k ; i_{-} M\right)=\frac{k}{k} H \quad k @ c A d(r ; c ;)\left(k ; r_{-} M\right)
$$

that we dierentiate once more to derive

$$
\begin{aligned}
& d^{2} H\left(\left(k ; i_{-} M_{0}\right) ;\left(\frac{k_{1}}{1} ;-M\right)\right) \\
& \begin{array}{ll}
0 & 1
\end{array} \\
& =k d @ c A\left(k ; i_{-} M\right) r_{;}^{2} ; \quad d @ c A\left(k ; i_{-} M\right)
\end{aligned}
$$

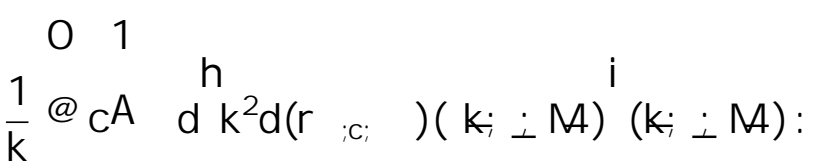

Now dierentiating (24) yields

$$
d\left(r_{; c ;}\right)\left(k_{;} ;-M\right)=\frac{1}{k} A @_{M^{-}}^{0} A
$$

which also implies

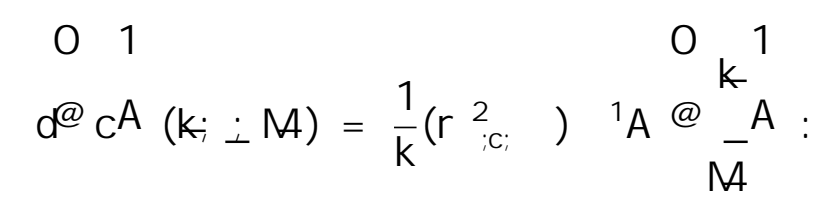

In turn

$$
\begin{aligned}
& \begin{array}{lllll}
0 & 0 & 0 & 0 & 1
\end{array} \\
& d(k A)\left(k ; i_{-} M\right)=B_{0} r \quad \cup Q(M) \quad M \quad 2 k k \quad k r \cup Q(M)^{\top}+k r \cup Q(M)^{\top} \xi \\
& M \quad 0 \quad k l_{N}
\end{aligned}
$$


so that

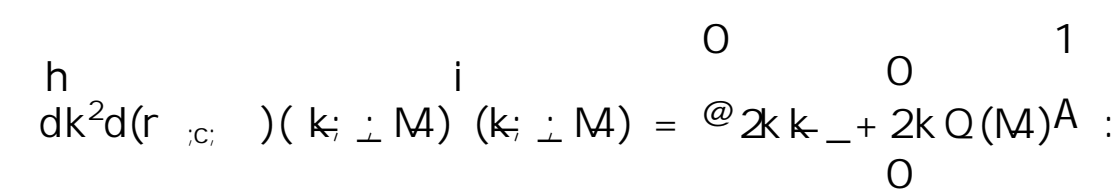

Inserting ( 31$)$ and (32) in (30) achieves the proof by identication of relevant symmetric matrices with corresponding quadratic forms since

$$
2 k_{-}+2 Q(M)=@_{M}^{k^{1}} B^{1} @_{-}^{0} A \text { : }
$$

Remark 8. Relation (29) leaves the possibility for $r_{k ; ;}^{2} \mathrm{M}$ to be denite without $\quad r_{; c ;}^{2}$ being so, and vice and versa. This could be of importance since any of those yields hyperbolicity of the modulated system and it was shown in [B GMR 16] that the negative signature of $r_{i ;}^{2}$; must be equal to $N$ modulo an even number for the underlying wave to be spectrally stable. Y et as already mentioned, in practice, this is hardly ever the case; see [Mie17] for numerous numerical experiments, and [BGMRar] and Remark 13 for the analysis of signatures in either one of the extreme regimes, small amplitude or large wavelength.

A s seen on the quasilinear form (28), the characteristic matrix of System (22) reads

$$
W:=\operatorname{Br} \underset{k ; i}{2} \mathrm{H} \text { : }
$$

We refer to $W$ in the sequel as the Whitham matrix of $(22)$. It can be rewritten using Equation (29) as

$$
W=\frac{1}{k} B A^{\top}\left(r_{; c ;}^{2}\right) \quad{ }^{1} A+C l_{N+2}:
$$

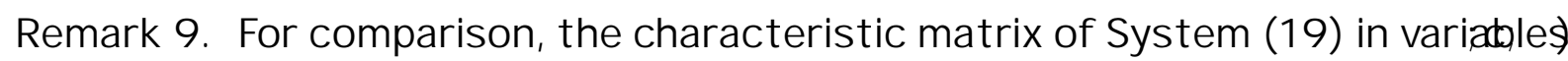
is

$$
\frac{1}{\mathrm{k}}\left(\mathrm{r}_{; \mathrm{i} ;}^{2}\right) \quad{ }^{1} \mathrm{~S}+\mathrm{Cl}_{\mathrm{N}+2}
$$

with

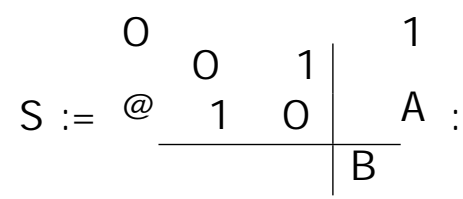

As follows from (31), ( $r \underset{; c ;}{2}$ ) ${ }^{1} A$ provides a change of basis between characteristic matrices. This may be checked directly thanks to the identity

$$
S=A B A^{\top}:
$$




\section{A symptotic expansions of parameters}

\subsection{Expansions of action derivatives}

Our study of extreme regimes hinges on asymptotic expansions of the action and its derivatives, obtained in [BGMRar] and that we partially recall here.

To conveniently write some of the coecients of the expansions, we rst make more explicit the prole equations (15)-(16) As in [BGMRar] we introduce the potential $\mathrm{W}(\mathrm{v} ; \mathrm{C} ;)$ dened in the case $\mathrm{N}=1$ by

$$
W(v ; c ;):=f(v) \quad \frac{1}{2} \frac{c}{b} v^{2} \quad v ;
$$

and in the case $\mathrm{N}=2$ by

$$
W(v ; c ;):=f(v) \quad \frac{1}{2}(v) g(v ; c ; 2)^{2} \quad \frac{c}{b} v g(v ; c ; 2) \quad 1 v \quad 2 g(v ; c ; 2)
$$

with

$$
g(v ; c ;):=\frac{1}{(v)} \frac{c}{b} v+\quad:
$$

The point is that $(15)-(15)$ s equivalently written

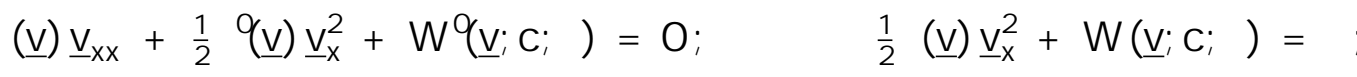

completed, in the case $\mathrm{N}=2$, with

$$
\underline{u}=g(\underline{v} ; c ; 2):
$$

We only consider non-degenerate limits. The nature of the non-degeneracy is made precise in the following set-up.

A ssumption 2. Harmonic limit Fix $\left({ }_{0} ; \underline{c}_{0} ;{ }_{0}\right) 2^{-}$such that there exists $\underline{v}_{0}>0$ such that

$$
{ }_{-0}=\mathrm{W}\left(\underline{\mathrm{v}}_{0} ; \underline{\mathrm{C}}_{0} ; \__{0}\right) ; \quad @ \mathrm{~W}\left(\underline{\mathrm{v}}_{0} ; \underline{\mathrm{C}}_{0} ; \__{0}\right)=0 ; \quad \mathrm{Q}^{2} \mathrm{~W}\left(\underline{\mathrm{v}}_{0} ; \underline{\mathrm{C}}_{0} ;{ }_{0}\right)>0:
$$

Then there exists a connected open neighborhood of $\left(\underline{C}_{0} ;{ }_{0}\right)$ and smooth functions $V_{0}$ :

! $(0 ; 1)$ and $0: \quad ! \mathrm{R}$ such that $\left(\mathrm{v}_{0} ; 0\right)\left(\underline{\mathrm{c}}_{0} ;{ }_{0}\right)=\left(\underline{\mathrm{v}}_{0} ;{ }_{0}\right)$ and for any $(\mathrm{c} ; \quad) 2$

$$
{ }_{0}(c ;)=W\left(v_{0}(c ;) ; c ;\right) ; \quad @ W\left(v_{0}(c ;) ; c ;\right)=0 ; \quad @^{2} W\left(v_{0}(c ;) ; c ;\right)>0 \text { : }
$$

M oreover one may ensure 4 that for some $r_{0}>0$

$$
\begin{aligned}
& r_{0}^{r_{0}}:=\left[\quad\left(0(c ;) ; 0(c ;)+r_{0}\right) f \quad(c ;) g\right. \\
& \text { (c; } 12
\end{aligned}
$$

\footnotetext{
${ }^{4} \mathrm{Up}$ to choosing the correct branch of parametrization and extending if necessary. Implicitly chosen consistently. 
and there exist $v_{2}$ and $v_{3}$ smooth maps dened on $\quad \begin{array}{ll}r_{0} \\ 0\end{array}$ such that for any $=(; C ; \quad) 2 \begin{aligned} & r_{0} \\ & 0\end{aligned}$,

$$
\begin{aligned}
0<\mathrm{v}_{2}() & <\mathrm{v}_{0}(\mathrm{c} ;)<\mathrm{v}_{3}() ; \quad=\mathrm{W}\left(\mathrm{v}_{2}() ; \mathrm{c} ;\right)=\mathrm{W}\left(\mathrm{v}_{3}() ; \mathrm{c} ;\right) ; \\
& @ \mathrm{~W}\left(\mathrm{v}_{2}() ; \mathrm{c} ;\right) \in 0 ; \quad @ \mathrm{~W}\left(\mathrm{v}_{3}() ; \mathrm{c} ;\right) \in 0 ; \\
& 8 \mathrm{v} 2\left(\mathrm{v}_{2}() ; \mathrm{v}_{3}()\right) ; \quad \in \mathrm{W}(\mathrm{v} ; \mathrm{c} ;):
\end{aligned}
$$

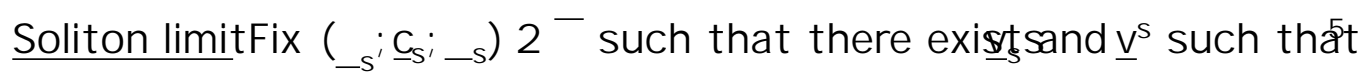

$$
\begin{aligned}
& 0<\underline{v}_{s}<\underline{v}^{s} ; \quad \quad \quad=W\left(\underline{v}_{s} ; \underline{C}_{s} ;-s\right)=W\left(\underline{v}^{s} ; \underline{c}_{s} ;-s\right) ; \\
& \text { @W }\left(\underline{\mathrm{v}}_{s} ; \underline{\mathrm{C}}_{s} ;{ }_{s}\right)=0 ; \quad Q_{\mathrm{W}}^{2} \mathrm{~W}\left(\underline{\mathrm{v}}_{s} ; \underline{\mathrm{C}}_{s} ;{ }_{s}\right)<0 \text {; } \\
& @ W\left(\underline{\mathrm{v}}^{\mathrm{s}} ; \underline{\mathrm{C}}_{s} ;-s\right) \in 0 ; \quad \text { and } 8 \mathrm{v} 2\left(\underline{\mathrm{v}}_{s} ; \underline{\mathrm{v}}^{\mathrm{s}}\right) ;{ }_{-} \in \mathrm{W}\left(\mathrm{v} ; \underline{\mathrm{C}}_{s} ;{ }_{s}\right) \text { : }
\end{aligned}
$$

Then there exists a connected open neighborhood of $\left(\underline{c}_{s} i_{s}\right)$ and smooth functions $v_{s}$ :

! $(0 ; 1), \mathrm{v}^{\mathrm{s}}:$ ! $(0 ; 1)$ and $\mathrm{s}:$ ! R such that $\left(\mathrm{v}_{s} ; \mathrm{v}^{\mathrm{s}} ; 0\right)\left(\underline{\mathrm{c}}_{s} ;-s\right)=\left(\underline{\mathrm{v}}_{s} ; \underline{\mathrm{v}}^{\mathrm{s}} ;{ }_{s}\right)$ and for any $(\mathrm{c} ; \quad) 2$

$$
\begin{array}{ll}
0<\mathrm{v}_{\mathrm{s}}(\mathrm{c} ;)<\mathrm{v}^{\mathrm{s}}(\mathrm{c} ;) ; & { }_{\mathrm{s}}(\mathrm{c} ;)=\mathrm{W}\left(\mathrm{v}_{\mathrm{s}}(\mathrm{c} ;) ; \mathrm{c} ;\right)=\mathrm{W}\left(\mathrm{v}^{\mathrm{s}}(\mathrm{c} ;) ; \mathrm{c} ;\right) ; \\
@ \mathrm{~W}\left(\mathrm{v}_{\mathrm{s}}(\mathrm{c} ;) ; \mathrm{c} ;\right)=0 ; & \mathrm{Q}^{2} \mathrm{~W}\left(\mathrm{v}_{\mathrm{s}}(\mathrm{c} ;) ; \mathrm{c} ;\right)<0 ;
\end{array}
$$

$@ W\left(\mathrm{v}^{\mathrm{s}}(\mathrm{c} ;) ; \mathrm{c} ;\right) \in 0 ; \quad$ and $8 \mathrm{v} 2\left(\mathrm{v}_{\mathrm{s}}(\mathrm{c} ;) ; \mathrm{v}^{\mathrm{s}}(\mathrm{c} ;)\right) ; \quad{ }_{\mathrm{s}}(\mathrm{c} ;) \in \mathrm{W}(\mathrm{v} ; \mathrm{c} ; \quad)$ :

M oreover one may ensure that for some $r_{0}>0$

$$
\begin{aligned}
& \underset{s}{r_{0}}:=\left[\begin{array}{lllll}
\left({ }_{s}(c ;)\right. & \left.r_{0} ; s(c ;)\right) f & (c ;) g
\end{array}\right. \\
& \text { (c; } 12
\end{aligned}
$$

and there exist $v_{1}, v_{2}$ and $v_{3}$ three smooth maps dened on ${\underset{s}{r}}_{s}^{r_{0}}$ such that for any = $(; c ; \quad) 2 \stackrel{r_{0}}{r_{0}}$,

$$
\begin{aligned}
0 & <\mathrm{v}_{1}()<\mathrm{v}_{\mathrm{s}}(\mathrm{c} ;)<\mathrm{v}_{2}()<\mathrm{v}_{3}()<\mathrm{v}^{\mathrm{s}}(\mathrm{c} ;) ; \\
& =\mathrm{W}\left(\mathrm{v}_{1}() ; \mathrm{c} ;\right)=\mathrm{W}\left(\mathrm{v}_{2}() ; \mathrm{c}_{;}\right)=\mathrm{W}\left(\mathrm{v}_{3}() ; \mathrm{c}_{;}\right) ;
\end{aligned}
$$

$@ W\left(v_{1}() ; c ;\right) \in 0 ; \quad @ W\left(v_{2}() ; c ;\right) \in 0 ; \quad @ W\left(v_{3}() ; c ;\right) \in 0 ;$

$8 \mathrm{v} 2\left(\mathrm{v}_{1}() ; \mathrm{v}_{2}()\right)\left[\left(\mathrm{v}_{2}() ; \mathrm{v}_{3}()\right) ; \quad \in \mathrm{W}(\mathrm{v} ; \mathrm{c} ;)\right.$ :

For all ( $c ; \quad) 2$, we consider

either $\quad 0:=(c ; \quad ; 0(c ;))$; or $s:=(c ; s(c ;))$;

which both belong to, and the corresponding harmonic limit $\left(\begin{array}{ll}!^{0^{0}} & 0\end{array}\right)$ and soliton limit $\left(!^{s^{0}} s\right)$. Actually it is more convenient and sucient to $x \quad(c ; \quad) 2$ and consider either $\quad{ }_{0}(\mathrm{c} ;)^{+}$or $\quad !{ }_{s}(\mathrm{c} ; \quad)$, provided one ensures local uniformity

${ }^{5}$ The choice that @W $\left(\underline{\mathrm{v}}_{s} ; \underline{\mathrm{c}}_{s} ;{ }_{s}\right)=0$ and @W $\left(\underline{\mathrm{v}}^{s} ; \underline{\mathrm{c}}_{s} ;{ }_{s}\right) \in 0$ instead of @W $\left(\underline{\mathrm{v}}_{s} ; \underline{\mathrm{c}}_{s} ;{ }_{s}\right) \in 0$ and @ $\mathrm{W}\left(\underline{\mathrm{v}}^{\mathrm{s}} ; \underline{\mathrm{c}}_{s} ;{ }_{s}\right)=0$ is arbitrary and purely made for the sake of clarity and deniteness. There is no loss of generality since one may go from one case to the other by rewriting the equations for $\underline{v}$ in terms of $\underline{v}$. 
with respect to $(\mathrm{C} ; \quad) 2$. By acting in this way, in [B GMRarl We derived asymptotic expansions in terms of two small parameters going to zero:

$$
:=\left(\begin{array}{ll}
v_{3} & v_{2}
\end{array}\right)=2
$$

in the harmonic limit and

$$
\%:=\frac{v_{2} \quad v_{1}}{v_{3} \quad v_{2}}
$$

in the soliton limit. These expansions are recalled below after a few preliminaries.

First, for the sake of concision, in the case $N=2$ we introduce notation $q(v ; c ;):=$ $Q(v ; g(v ; c ;))$, still with $g(v ; c ; \quad)=((c=b) v+)=(v)$. Note that in the sequel $g$ and $q$ are evaluated at $=2$, the second component of . For convenience we adopt a similar convention in the case $N=1$ with merely $q(v):=Q(v)$. In the statement that follows, we omit to write the dependence $\mid$ if any | of these functions on the parameters ( in order to shorten formulas a little bit and stress symmetry between cases $\mathrm{N}=1$ and $\mathrm{N}=2$. We also make use of the symmetric matrix $\mathrm{S}$ dened in (34).

Now we introduce a set of vectors that are crucially involved in the above-mentioned asymptotic expansions, and provide associated key cancellations proved in [BGMRar, Lemma 1].

Proposition 4 ([BGMRar $]$ ). For both indices $\mathrm{i}=0$ and $\mathrm{i}=\mathrm{s}$ we introduce the following vectors of $\mathrm{R}^{\mathrm{N}+2}$ : for $\mathrm{N}=2$

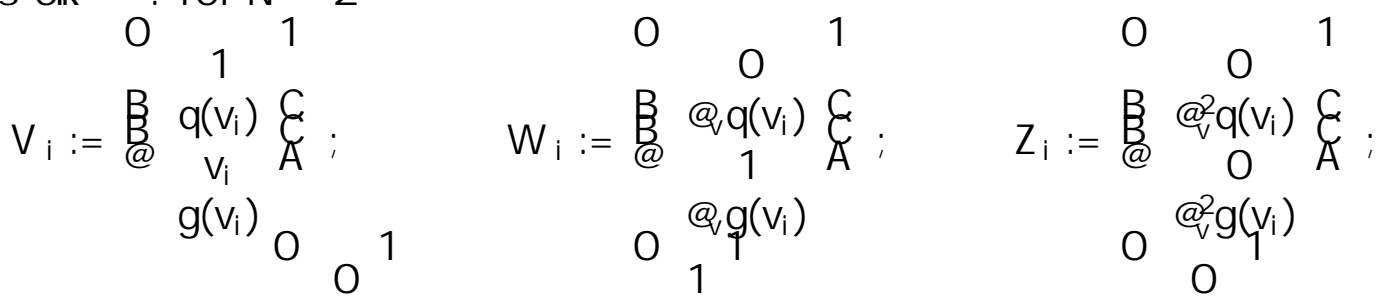

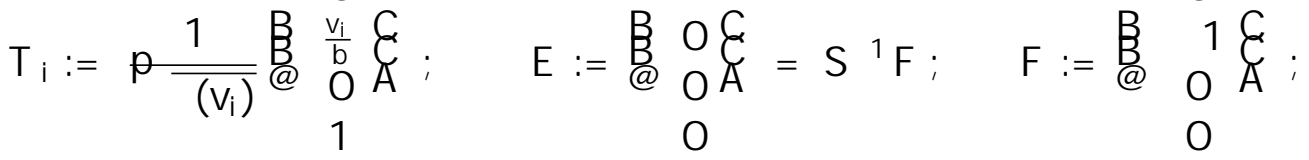

and for $\mathrm{N}=1$

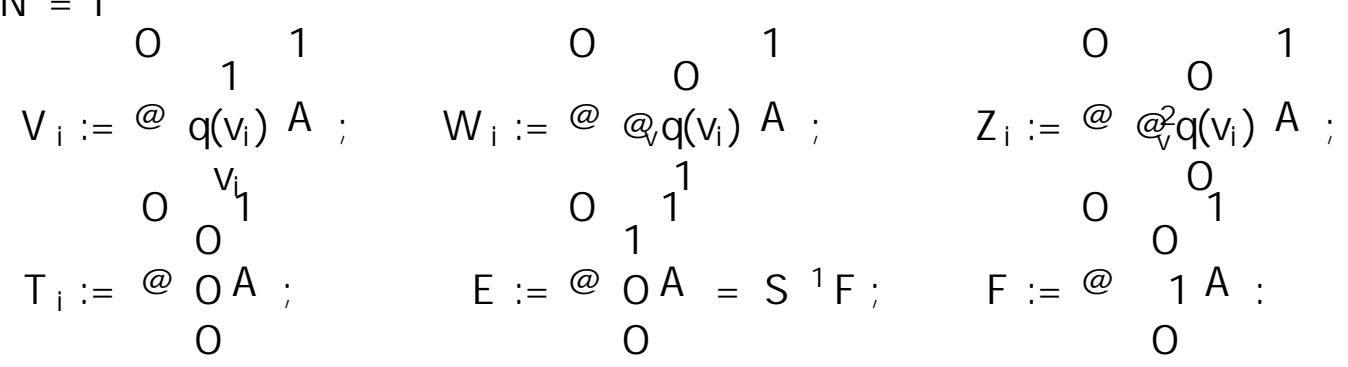

These vegctors are such that

$$
\begin{aligned}
& \mathrm{V}_{\mathrm{i}} \mathrm{S}^{1} \mathrm{~V}_{\mathrm{i}}=0 ; \mathrm{V}_{\mathrm{i}} \mathrm{S}^{1} \mathrm{~W}_{\mathrm{i}}=0 ; \mathrm{V}_{\mathrm{i}} \mathrm{S}^{1} \mathrm{~T}_{\mathrm{i}}=0 ; \\
& \mathrm{V}_{\mathrm{i}} \mathrm{S}^{1} \mathrm{Z}_{\mathrm{i}}=\mathrm{W}_{\mathrm{i}} \mathrm{S}^{1} \mathrm{~W}_{\mathrm{i}} ; \quad \mathrm{T}_{\mathrm{i}} \mathrm{S}^{1} \mathrm{~T}_{\mathrm{i}}=0 ; \quad \mathrm{T}_{\mathrm{i}} \mathrm{S}^{1} \mathrm{Z}_{\mathrm{i}}=0 ; \\
& \mathrm{E} \mathrm{V}_{\mathrm{i}}=1 ; \quad \mathrm{E} \mathrm{W}_{\mathrm{i}}=0 ; \quad \mathrm{E} \mathrm{Z}_{\mathrm{i}}=0 ; \quad \mathrm{E} \quad \mathrm{T}_{\mathrm{i}}=0:
\end{aligned}
$$


At last, we introduce the Boussinesq moment of instability involved in solitary wave limits. We stress that it is both convenient and classical to parameterize solitary wave proles $\underline{U}^{s}$ not by $(c ; \quad)$ but by $\left(c ; U_{s}\right)$ with $U_{s}$ the corresponding endstate. The associated is then recovered through

$$
={ }_{s}\left(C ; U_{s}\right):=r \quad u(H+C Q)\left(U_{s} ; 0\right)
$$

and $s$ is simply obtained as

$$
{ }_{s}=(H+c Q)\left(U_{s} ; 0\right)+r_{U}(H+C Q)\left(U_{s} ; 0\right) U_{s}:
$$

The B oussinesq moment of instability is then dened as

$$
\begin{aligned}
& M\left(c ; U_{s}\right)={ }_{1}^{Z+1}\left(H\left[\underline{U}^{s}\right]+c Q\left(\underline{U}^{s}\right)+s \underline{U}^{s}+s\right) d \\
& \quad \begin{array}{l}
Z+1 \\
=
\end{array} \quad(H+c Q)\left[\underline{U}^{s}\right] \quad(H+c Q)(U s ; 0) r \quad u(H+c Q)(U s ; 0) \quad\left(\underline{U}^{s} \quad U_{s}\right) d:
\end{aligned}
$$

Note that, since $(H+C Q)\left[\underline{U}^{s}\right]+{ }_{s}=0$, we do have

$$
@ M\left(c ; U_{s}\right)=\underbrace{Z+1}_{1} Q\left(\underline{U}^{s}\right) Q\left(U_{s}\right) r \quad u Q\left(U_{s}\right)\left(\underline{U}^{s} \quad U_{s}\right) d
$$

The following statement gathers elements from [BGMRar, Theorems 4 and 5] and their proofs.

Theorem 2 ([BGMRarl). Under A ssumptions -7/7we have the following asymptotics for the action derivatives.

Harmonic limit There exist real numbers $a_{0}, b_{0}$ and a positive number $c_{0} \mid$ depending smoothly on the parameters $(c ;) \mid$ such that when goes to zero

$$
\begin{aligned}
& \frac{4 c_{0}}{0} r_{; c ;}=4 \quad c_{0} V_{0}+\left(a_{0} V_{0}+b_{0} W_{0}+c_{0} Z_{0}\right)^{2}+0\left({ }^{4}\right) \\
& \frac{1}{0} r_{; c ;}^{2}=a_{0} V_{0} \quad V_{0}+b_{0}\left(V_{0} \quad W_{0}+W_{0} \quad V_{0}\right) \quad T_{0} \quad T_{0} \\
& +2 c_{0} W_{0} \quad W_{0}+c_{0}\left(\begin{array}{lll}
V_{0} & Z_{0}+Z_{0} & V_{0}
\end{array}\right)+0\left({ }^{2}\right)
\end{aligned}
$$

where 0 denotes the harmonic period at $v_{0}$, that is, $\quad 0=P \frac{P}{\left.\left(v_{0}\right)=Q^{2} W\left(v_{0} ; c ; 1\right)\right)}$. Soliton limit There exist real numbers $a_{s}, b_{s}$, positive numbers $c_{s}, h_{s}$, a vector $X_{s}$ and $a$ symmetric matrix $O_{s} \mid$ depending smoothly on the parameters $(c ;) \mid$ such that

(40) $\underset{s}{-} r_{; c_{i}}=v_{s} \ln \% \quad X_{s}+\frac{\%}{2} v_{s} \frac{1}{2 h_{s}}\left(a_{s} V_{s}+b_{s} W_{s}+c_{s} Z_{s}\right) \% \ln \%+0 \%$ 
(41)

$$
\begin{aligned}
-r_{i c ;}^{2}= & h_{s} \frac{1+\%}{\%} V_{s} \quad v_{s}+\left(a_{s} V_{s} \quad V_{s}+b_{s}\left(V_{s} \quad W_{s}+W_{s} \quad v_{s}\right)\right) \ln \% \\
& +\left(T_{s} T_{s}+2 c_{s} W_{s} \quad W_{s}+c_{s}\left(Z_{s} \quad V_{s}+V_{s} \quad Z_{s}\right)\right) \ln \% \\
& +0_{s}+0 \% \ln \%
\end{aligned}
$$

when \%goes to zero, where $s$ denotes the harmonicpperiod at $v_{s}$ of waves associated with the opposite 'capillarity' coecient, that is, $\left.s:=\quad\left(v_{s}\right)=@^{2} W\left(v_{s} ; c ;\right)\right)$. In addition, we hav $\AA^{6}$

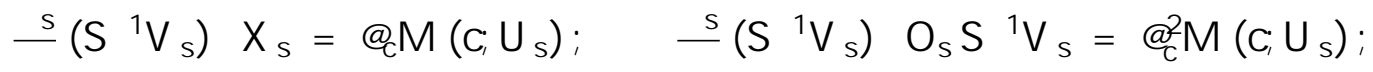

where $U_{s}=v_{s}$ in the case $N=1$ and $U_{s}=\left(v_{s} ; g\left(v_{s}\right)\right)$ in the case $N=2$.

In the latter theorem and el sewhere in the present paper, for any two vectors $\quad \mathrm{V}$ and $\mathrm{W}$ in $\mathrm{R}^{\mathrm{d}}$, thought of as column vectors, $\mathrm{V} \quad \mathrm{W}$ stands for the rank-one, square matrix of sized

$$
\mathrm{V} \quad \mathrm{W}=\mathrm{VW}^{\mathrm{T}}
$$

whatever $d$.

Observing that the matrices involved in the expansions of $r_{; ;}^{2}$ in both the harmonic and the soliton limit have similar structures, we nd useful to have at hand the following set of algebraic properties, which are either simple reformulations of relations in

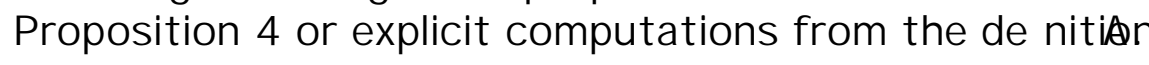

Corollary 3. Case $\mathrm{N}=1$ With

$$
P_{i}:=S^{1} \quad F_{i} \quad V_{i} \quad W_{i} ;
$$

we have

$$
\mathrm{D}_{\mathrm{i}}:=\mathrm{P}_{\mathrm{i}}^{\top} \mathrm{S} \mathrm{P}_{\mathrm{i}}=\mathrm{B}^{1}=\begin{array}{lll|ll}
0 & 0 & 1 & 0 & \\
& 0 & 0 & 0 & \mathrm{~A} \\
\hline 0 & 0 & \mathrm{~b}^{1}
\end{array} ;
$$

\footnotetext{
${ }^{6}$ This comes from the proof of Theorem 5 in [BGMRar], the statement of which lacked the prefactor $\underline{s}$ in the relation between $\oint_{C}^{2} M$ and the \%-term in the expansion of $r_{; c ;}^{2}$. We have corrected this omission in (42).
} 


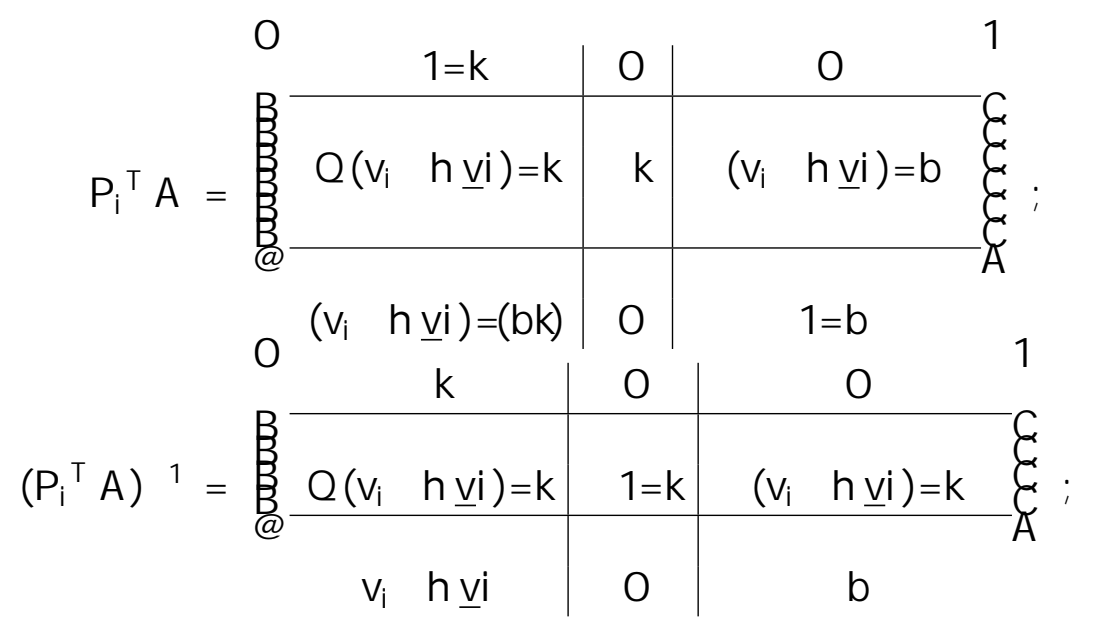

and, for any real numbers $(a ; b ; c ; m)$

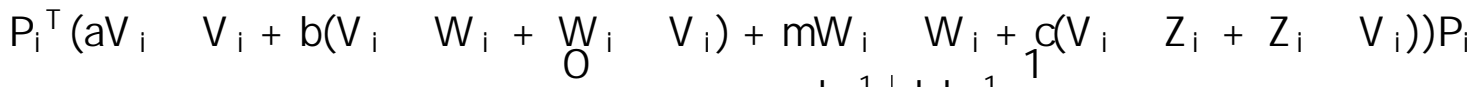

$$
\begin{aligned}
& =@ \begin{array}{cc|cc}
a & c b^{1} & b b^{1} \\
c b^{1} & 0 & 0 & A
\end{array} \text { : }
\end{aligned}
$$

Case $\mathrm{N}=2$ With

$$
\begin{aligned}
& P_{i}:=S^{1} \quad F_{i} \quad V_{i} T_{i} W_{i} ; \\
& i:=T_{i} S{ }^{1} W_{i}=\frac{p}{b^{p} \frac{1}{(v)}} ; \\
& W_{i}:=W_{i} S{ }^{1} W_{i}=\frac{2 g_{v}\left(V_{i}\right)}{b} ; \\
& i:=Z_{i} S{ }^{1} W_{i}=\frac{g_{v v}\left(V_{i}\right)}{b} ;
\end{aligned}
$$

and

$$
A_{i}:=\begin{array}{ccc}
0 & 1= & p \\
1 & g_{v}\left(v_{i}\right)
\end{array}=\begin{array}{lll}
0 & b_{i} \\
1 & \frac{b}{2} w_{i}
\end{array} ; ;
$$

we have

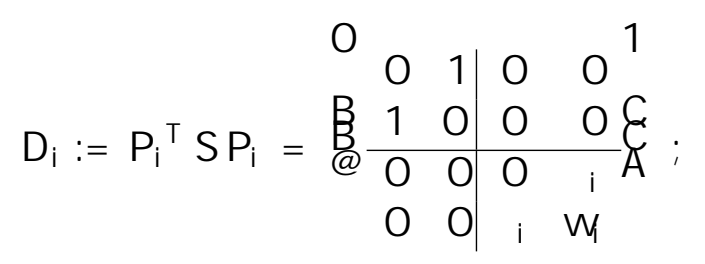




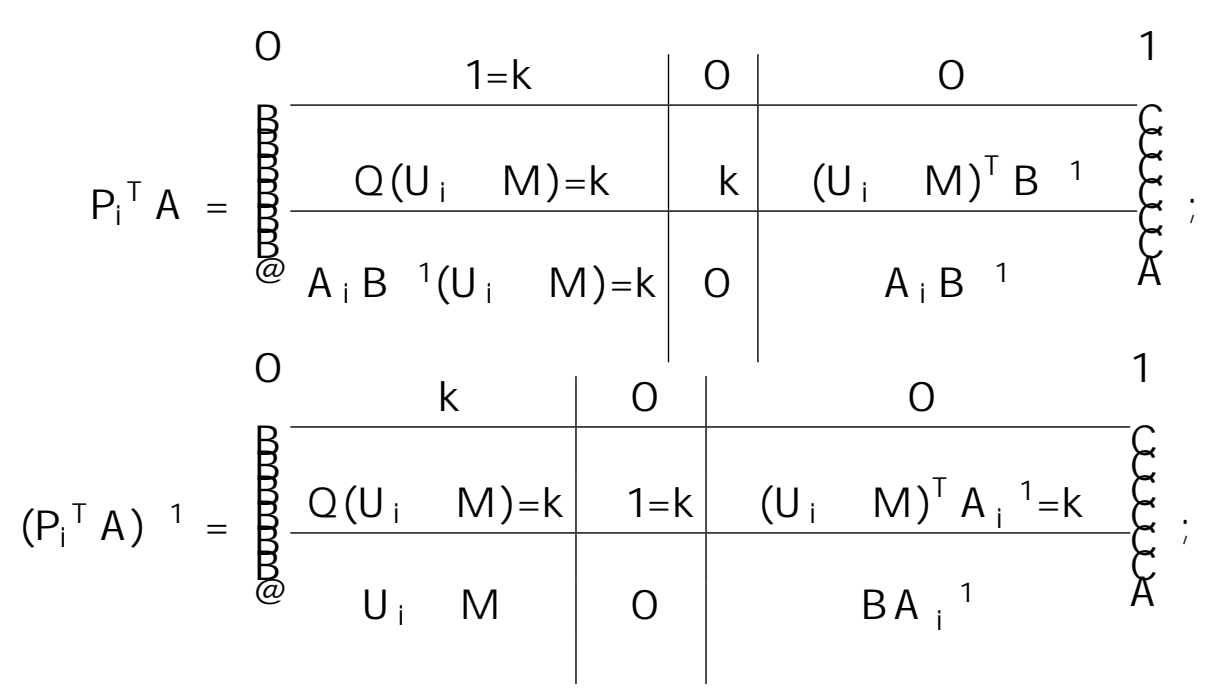

and, for any real numbers $(a ; b ; c ; m ; n)$

$\left.P_{i}^{\top}\left(\begin{array}{lllllll}a V_{i} & V_{i}+b_{0} V_{i} & W_{i}+W_{i} & V_{i}\end{array}\right)+m W_{i} \quad W_{i}+c\left(V_{l^{i}} \quad Z_{i}+Z_{i} \quad V_{i}\right)+n T_{i} \quad T_{i}\right) P_{i}$

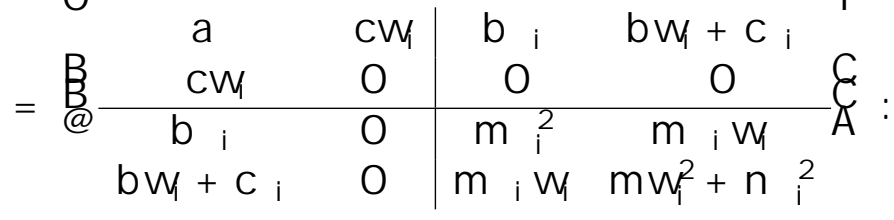

For later reference, let us point out here that in any case

$$
D_{i}:=P_{i}^{\top} S P_{i}:
$$

To unify cases $N=1$ and $N=2$, it is also useful to extend to $N=1$ denitions in (45) and to set $A_{i}=1$ when $\mathrm{N}=1$.

\subsection{Expansions of modulated variables}

By (18) we have = @ so that we readily obtain expansions for the period by projecting ( $\overline{38})$ and $(40)$ onto their rst component $\mid$ which amounts to taking the inner product with $E$. This gives

$$
\begin{aligned}
& =\quad 01+\frac{a_{0}}{4 c_{0}}{ }^{2}+0\left({ }^{4}\right) ; \\
& =\stackrel{s}{ }=\ln \% \quad E \quad X_{s}+\frac{\%}{2} \quad \frac{a_{s}}{2 h_{s}} \% \ln \%+0 \% \quad ; \quad \% ! \quad 0 ;
\end{aligned}
$$

from which we can of course infer expansions for the local wave number $k=1=$

$$
\begin{array}{lllll}
k=k_{0} & 1 & \frac{a_{0}}{4 c_{0}}{ }^{2}+0\left({ }^{4}\right) ; & ! & 0 ; \\
k=- & \frac{1}{\ln \%}+\frac{E X_{s}}{\left(\ln \%^{2}\right.} & \frac{\%}{2(\ln \%)^{2}} & \frac{\left(E X_{s}\right)^{2}}{\left(\ln \%^{3}\right.}+\frac{\left(E X_{s}\right) \%}{(\ln \%)^{3}}+0 \frac{\%}{\ln \%} ; \% ! & 0 ;
\end{array}
$$


where $\mathrm{k}_{0}=1=0$.

Thanks to (18) again, the projections of (38) and (40) onto their intermediate and last components together with the expansions of $k$ yield expansions for the mean values $\mathrm{hQ}(\underline{\mathrm{U}}) \mathrm{i}$ and $\mathrm{hU} \mathrm{i}$. To carry this out, it is convenient to introduce the $\mathrm{N}(\mathrm{N}+2)$ matrix

$$
I:=@ \begin{array}{ccc|}
0 & & 1 \\
I_{N} & A
\end{array}
$$

of the projection onto last components, and to observe that taking the projection on the second component of vectors in $\mathrm{R}^{\mathrm{N}+2}$ amounts to taking the inner product with $\mathrm{F}$. We also recall that $U_{0}:=I V_{0}$ and $U_{s}:=I V_{s}$.

Regarding the expansions of the mean value $M=h \mathrm{U} i$ we get from (38) that

$$
\begin{aligned}
M & =0 \\
& =1 \quad U_{0}+\frac{1}{4 c_{0}}\left(a_{0} U_{0}+b_{0} I W_{0}+c_{0} I Z_{0}\right)^{2}+O\left({ }^{4}\right) \\
& \left.=U_{0}+Y_{0}+{ }^{4}\right) \quad U_{0}+\frac{1}{4 c_{0}}\left(a_{0} U_{0}+b_{0} I W_{0}+c_{0} I Z_{0}\right)^{2}+O\left({ }^{4}\right) ;
\end{aligned}
$$

when goes to zero, with

$$
Y_{0}:=\frac{1}{4 c_{0}}\left(b_{0} I W_{0}+c_{0} I Z_{0}\right)
$$

and from (40) that

$$
\begin{aligned}
& M=-s \quad U_{s} \ln \% \quad I X_{s}+\frac{\%}{2} U_{s} \quad \frac{1}{2 h_{s}}\left(a_{s} U_{s}+b_{s} I W_{s}+c_{s} I Z_{s}\right) \% \ln \%+0 \% \\
& =\frac{1}{\ln \%}+\frac{E X_{s}}{\left(\ln \phi^{2}\right.} \quad \frac{\%}{2\left(\ln \phi^{2}\right.} \quad \frac{\left(E X_{s}\right)^{2}}{\left(\ln \phi^{3}\right.}+\frac{\left(E X_{s}\right) \%}{\left(\ln \phi^{3}\right.}+0 \frac{\%}{\ln \%} \\
& U_{s} \ln \% \quad I X_{s}+\frac{\%}{2} U_{s} \quad \frac{1}{2 h_{s}}\left(a_{s} U_{s}+b_{s} I W W_{s}+c_{s} I Z_{s}\right) \% \ln \%+0 \% \\
& =U_{s}+\frac{Y_{s}}{\ln \%} \quad \frac{E}{\left(\ln \phi_{s}^{2}\right.} Y_{s}+\frac{\%}{2\left(\ln \phi^{2}\right.} Y_{s} \quad \frac{\left(E X_{s}\right) \%}{(\ln \%)^{3}} I X_{s}+0 \% \text {; }
\end{aligned}
$$

when \%goes to zero, with

$$
Y_{S}:=I X_{S} \quad\left(E \quad X_{S}\right) U_{S}:
$$

N ow that we have necessary pieces of notation, we gather in the following the behaviors found here above for ( $k$; hU i ) with the expansions proved below for . 
Corollary 4. Under A ssumptions -7 and with notation from Theorem 2, $\square$ (48) and (49) we have Harmonic limit

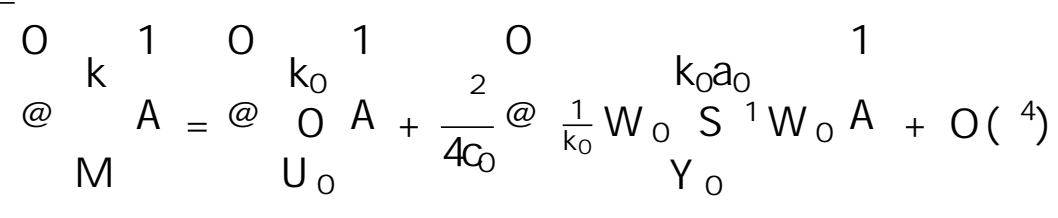

when goes to zero.

$\underline{\text { Soliton limit }}$

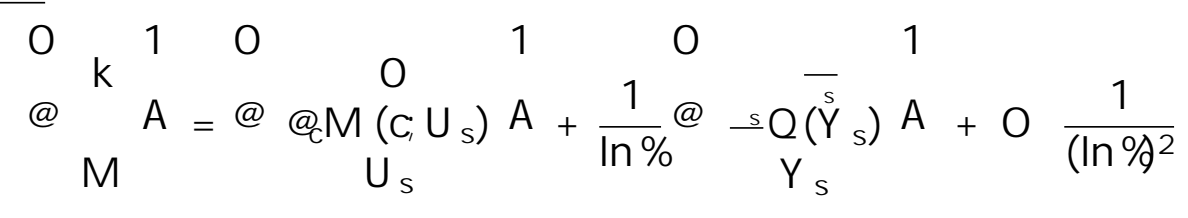

when $\%$ goes to zero.

Remark 10. One can always look at the soliton limit as being the limit when $k$ goes to zero and Corollary $\Psi$ n particular contains in this regime

$$
=@ M\left(c ; U_{s}\right)+O(k) ; \quad h \underline{U} i=U_{s}+O(k):
$$

Likewise, assuming that

$$
\mathrm{W}_{0}:=\mathrm{W}_{0} \quad \mathrm{~S}^{1} \mathrm{~W}_{0}
$$

is nonzero, we can equivalently look at the harmonic limit as the limit goes to zero and then

$$
\mathrm{k}=\mathrm{k}_{0}+\mathrm{O}(\mathrm{l}) ; \quad \underline{\mathrm{U} i}=\mathrm{U}_{0}+\mathrm{O}(\mathrm{l}):
$$

Remark 11. As already observed in [BGMRarl] we can check in practical cases that $\mathrm{W}_{0}=\mathrm{W}_{0} \mathrm{~S}^{1} \mathrm{~W}_{0} \in 0$. Indeed, $\mathrm{W}_{0} \quad \mathrm{~S}^{1} \mathrm{~W}_{0}=1=b$ in the case $\mathrm{N}=1$ and in the case $\mathrm{N}=2, \mathrm{~W}_{0} \quad \mathrm{~S}^{1} \mathrm{~W}_{0}=2 @ g\left(\mathrm{~V}_{0}\right)=\mathrm{b}$ is nonzero both when is constant $\mid$ which is the case for the Euler\{Korteweg system in mass Lagrangian coordinates $\mid$ and $\quad c \in 0$ and when is linear in $v \mid$ which is the case for the Euler\{Korteweg system in Eulerian coordinates $\mid$ and $\quad 2 \in 0$. We stress that the latter conditions are exactly the same conditions encountered in Proposition 2 Where the sign of was investigated. In particular as pointed out in Remark 4 [both conditions are conjugated by the passage between mass Lagrangian and Eulerian formulations.

Proof. The only thing left is to expand our variable . . In order to do so, by using (18) we can conveniently write it as

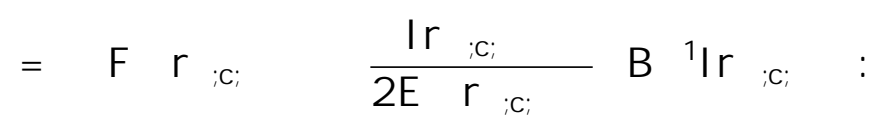


From (38) and Proposition 37 we obtain

$$
\begin{aligned}
& -\tau_{0} Q\left(U_{0}\right)+\frac{1}{4 c_{0}}\left(a_{0} Q\left(U_{0}\right) \quad b_{0} F \quad W_{0} \quad c_{0} F \quad Z_{0}\right)^{2}+0\left({ }^{4}\right) \\
& \frac{1}{1+\frac{a_{0}}{4 c_{0}}{ }^{2}+O\left({ }^{4}\right)} Q\left(U_{0}+\frac{1}{4 c_{0}}\left(a_{0} U_{0}+b_{0} I W_{0}+c_{0} I Z_{0}\right)^{2}+O\left({ }^{4}\right)\right) \\
& =Q\left(U_{0}\right)+\frac{1}{4 c_{0}}\left(a_{0} Q\left(U_{0}\right) \quad b_{0} F \quad W_{0} \quad c_{0} F \quad Z_{0}\right)^{2}+O\left({ }^{4}\right) \\
& \frac{1}{1+\frac{a_{0}}{4 c_{0}}{ }^{2}+0\left({ }^{4}\right)} Q\left(U_{0}\right)+U_{0} B^{1} \frac{1}{4 c_{0}}\left(a_{0} U_{0}+b_{0} I W_{0}+c_{0} I Z_{0}\right)^{2}+0\left({ }^{4}\right)
\end{aligned}
$$

in which there are some simplications because by (37) $\square$

$$
\begin{aligned}
\mathrm{F} \mathrm{W}_{0}+\mathrm{U}_{0} \mathrm{~B}{ }^{1} \mathrm{I} \mathrm{W}_{0} & =\mathrm{V}_{0} \mathrm{~S}^{1} \mathrm{~W}_{0}+\mathrm{Q}\left(\mathrm{U}_{0}\right) \mathrm{E} \mathrm{W}_{0}=0 ; \\
\mathrm{F} \mathrm{Z}_{0}+\mathrm{U}_{0} \mathrm{~B}{ }^{1} \mathrm{I} \mathrm{Z}_{0} & =\mathrm{V}_{0} \mathrm{~S}^{1} \mathrm{Z}_{0}+\mathrm{Q}\left(\mathrm{U}_{0}\right) \mathrm{E} \mathrm{Z}_{0}=\mathrm{W}_{0} \mathrm{~S}^{1} \mathrm{~W}_{0}:
\end{aligned}
$$

So we eventually nd that

$$
=\frac{W_{0} S^{1} W_{0}}{4 c_{0} k_{0}}+0\left({ }^{4}\right):
$$

Likewise, from (40) and Proposition 37 we get

$$
\begin{aligned}
-\bar{s}_{\mathrm{s}}= & \mathrm{Q}\left(\mathrm{U}_{\mathrm{s}}\right) \ln \%+\mathrm{F} \mathrm{X}_{\mathrm{s}}+0 \% \\
& +\frac{\ln \% \mathrm{U}_{\mathrm{s}}+\frac{\operatorname{lX} \mathrm{s}}{\ln \%}+0 \frac{\%}{\ln \%}}{1+\frac{\mathrm{EX}}{\ln \%}+0 \frac{\%}{\ln \%}} \mathrm{~B}^{1} \quad \mathrm{U}_{\mathrm{s}}+\frac{\operatorname{IX} \mathrm{X}_{\mathrm{s}}}{\ln \%}+0 \frac{\%}{\ln \%} ;
\end{aligned}
$$

which eventually simplies into

$$
\tau_{s}=F \quad X_{s}+U_{s} \quad B^{1} I_{s} \quad Q \quad\left(U_{s}\right)\left(\begin{array}{ll}
E & X_{s}
\end{array}\right)+\frac{Q\left(Y_{s}\right)}{\ln \%}+0 \frac{1}{(\ln \%)^{2}} ;
$$

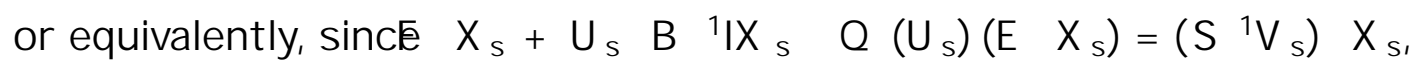

$$
=@ M\left(c ; U_{s}\right)+\frac{{ }_{s} Q\left(Y_{s}\right)}{\ln \%}+0 \frac{1}{(\ln \%)^{2}}
$$

thanks to (42)

\subsection{Extending the parametrization}

We can even go further and show that ( $k ; ; \quad M)$ are 'good' variables up to the limits $k=0$ and $=0$. 
Theorem 3. Under A ssumptions --2 and with notation from Theorem 2 we have Harmonic limit The continuous extension of

$$
\left({ }^{2} ; \mathrm{c} ;\right) 7 !(\mathrm{k} ; \quad \mathrm{M})
$$

or equivalently of

$$
\text { ( o(c; ); c; ) 7! (k; M ) }
$$

to $\mathrm{f} 0 \mathrm{~g}$ denes a $C^{1}$ map in a connected open neighborhood (in $R_{+}$) of $f 0 g$, which, provided that $]^{7} \mathrm{w}_{0}$ does not vanish on , is also a $\mathrm{C}^{1}$-dieomorphism. Soliton limit The continuous extension of the map

$$
\left(\frac{1}{\ln \%} ; \quad\right) 7 !(k ; ; M)
$$

or equival ently of the map

$$
\left.\left(\frac{1}{\ln (s(c ;)} ; c ;\right)\right) 7 !\left(k_{;} ; M\right)
$$

to $\mathrm{f} \mathrm{g}$ denes a $C^{1}$ map in a connected open neighborhood (in $R_{+} \quad$ ) of $\mathrm{f} 0 \mathrm{~g}$, which, provided that, for any $(c ;)_{2} 2, @_{2}^{2} M\left(c ; U_{s}(c ;)\right) \in 0$, is also a $C^{1}$-dieomorphism.

Proof. Expansions ( $\overline{50}$ ) and (51) show that the maps under consideration possess continuous extensions. To prove that these extensions are $C^{1}$, we only need to prove that their Jacobian maps also extend continuously to $f \quad 0 \mathrm{~g}$. A fter that, by the Inverse Function Theorem, the proof will be achieved provided we also derive from extra assumptions that at any point of $f \quad \mathrm{~g}$ the limit of the Jacobian map is nonsingular. In both limits our starting point is (31), that yields

$$
r_{; c ;} \stackrel{0}{k_{M}^{1}} A=k\left(r_{i c ;}^{2}\right)\left(A^{\top}\right)^{1} ;
$$

with $\mathrm{A}$ as in Proposition 3.

In the harmonic limit we set $:=2$ and observe that the chain rule yields follows from

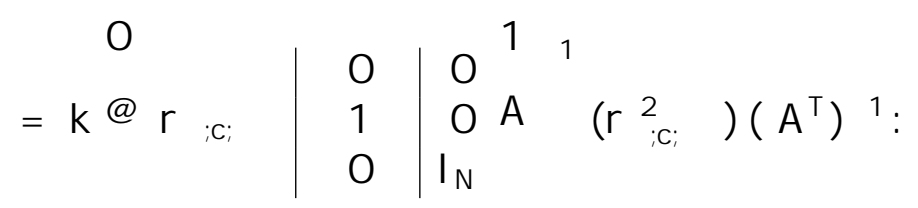

Since $k$ has a nonzero limit $k_{0}$, it is trivial to check that both $k$ and $A$ admit invertible limits when ! 0 . To deal with the factor involving $r_{; c}$; we extract from [BGMRar, Proposition 4], expressed in our current notation,

$$
r_{i c_{i}}=4 c_{0} V_{0}+O\left({ }^{2}\right)
$$

\footnotetext{
${ }^{7}$ See Remark 1.
} 
that implies readily that

$$
@ r_{; c ;} \quad \begin{array}{l|l|ll}
0 & 0 & 1 \\
1 & 0 & A \\
0 & I_{N} &
\end{array}
$$

possesses an invertible limit. At last, the fact that $\quad r_{;}^{2}$; possesses a limit when $\quad ! \quad 0$ is a direct consequence of (39). The invertibility of the corresponding limit when $w_{0}$ (dened in (52)) is nonzero follows from straightforward computations based on the limit of $\mathrm{P}_{0}{ }^{\top}\left(\mathrm{r}_{; c_{i}}^{2}\right) \mathrm{P}_{0}$ obtained from Corollary 3 .

M ore delicate is the soliton limit, in which all matrices involved in (53) blow up. To begin with we set $:=1=\ln \%$ and extract from [BGMRar, Proposition 5]

$$
r_{; c ;}=\frac{h_{s}}{\left(\% \ln \%^{2}\right.}\left(\left(1+\frac{3}{2} \% V_{s}+0(\%)\right) ;\right.
$$

To make the most of computations already carried out in Corollary 3 , we use the factorization

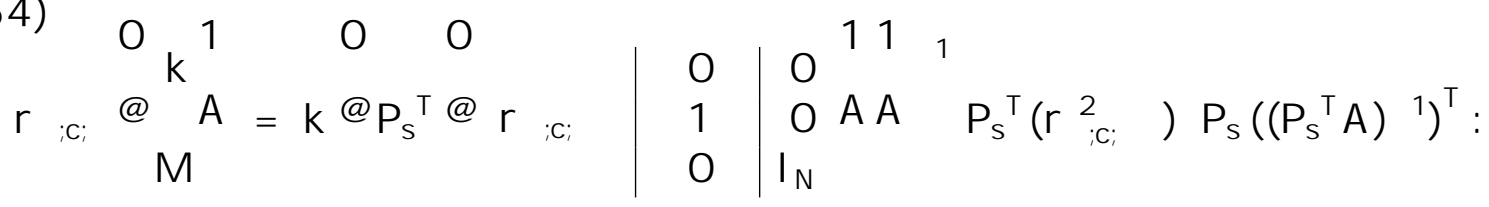

stemming from the chain rule. To do so, rst we observe that

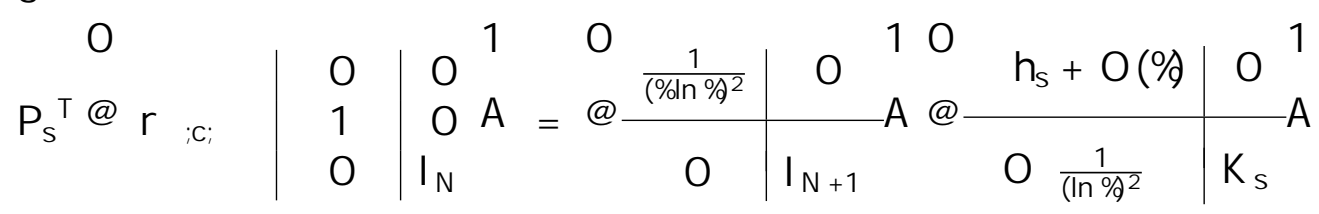

with

$$
K_{S}=0 \mid I_{N+1} \quad P_{S}^{\top} @ \frac{0}{I_{N+1}} A
$$

easily seen to be invertible so that the last matrix in (55) ppssesses an invertible limit when $\%$ ! 0 . Now we stress that

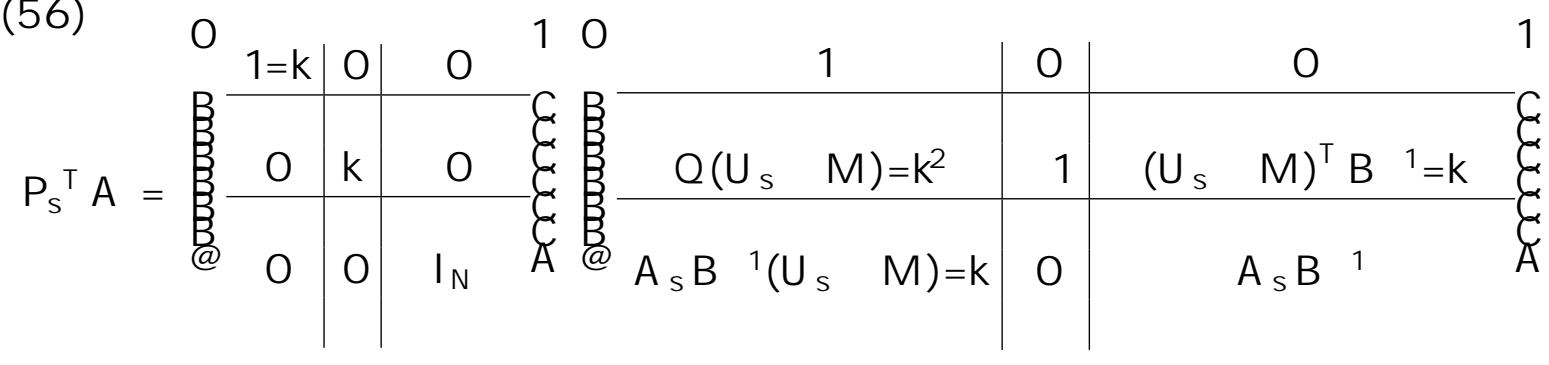

and that it follows from (51) that the last matrix in (56) possesses an invertible limit when $\%$ ! 0 . 
Combining (54)-(55)-(56) with (51) reduces the issue to the inspection of the matrix

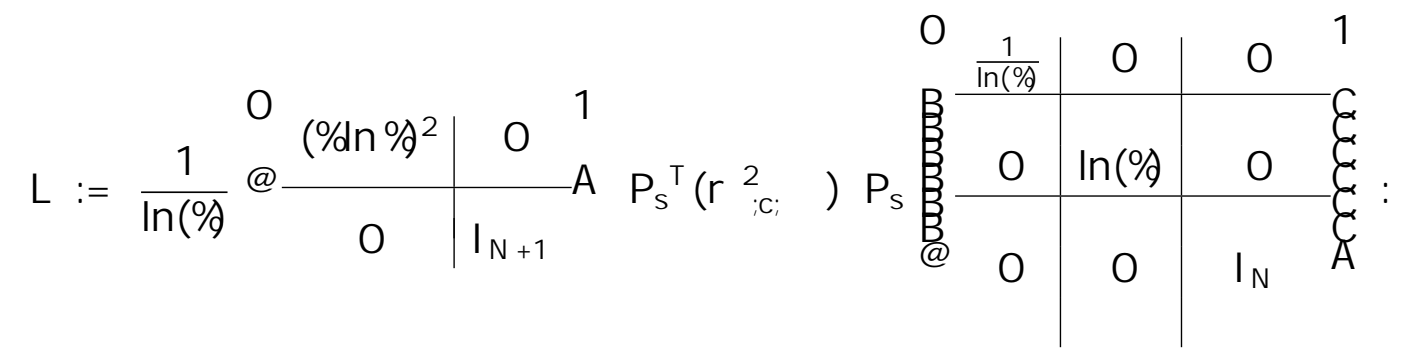

It follows from Theorem 2 and Corollary 3 that, when $\mathrm{N}=2$

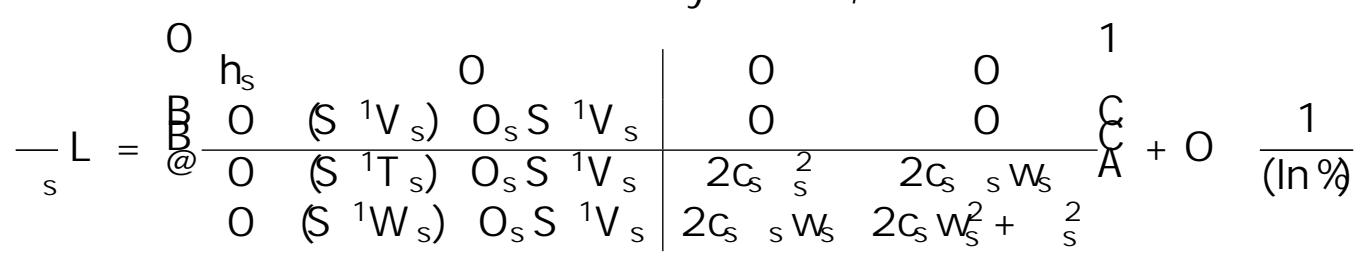

while when $\mathrm{N}=1$

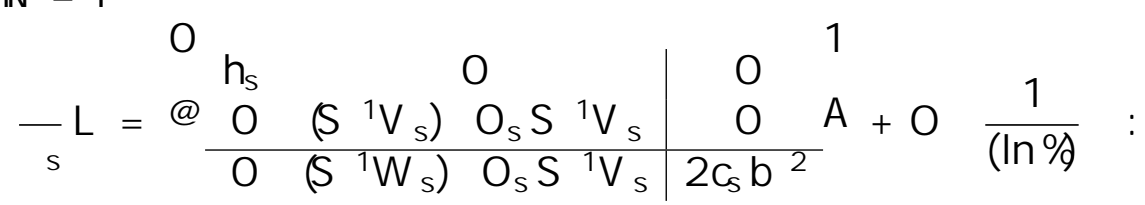

This implies that $L$ possesses a limit when $\%$ ! 0 and that this limit is invertible provided that $\left(S^{1} V_{s}\right) O_{s} S^{1} V_{s} \in 0$, and nishes the proof.

\section{A symptotics of the modulation system}

\subsection{Extending the averaged Hamiltonian}

Our goal is now to show that the averaged Hamiltonian $\mathrm{H}$ extends as a $\mathrm{C}^{2}$ function of $(\mathrm{k} ;$; $\mathrm{M})$ both to the zero-amplitude regime $\quad=0$ and to the zero-wavelength regime $k=0$.

Under natural assumptions required by Theorem 1, t tis quite elementary, by using the denition of $\mathrm{H}$ and relations (21), to check that $\mathrm{H}$ does extend as a $\mathrm{C}^{1}$ map both to $=0$ and to $\mathrm{k}=0$. This is al ready sucient to take the relevant limits of the conservative form (22) of the modulated system. Y et to ensure that hyperbolic properties of the limiting system do transfer to the original ones in relevant regimes one needs to be able to take limits in the quasilinear form (28) hence to prove the $C^{2}$ extension property we discuss now.

To state the following theorem in a precise way, let us denote, in the harmonic limit, as 0 the image of by $(c ;) 7 !\left(\mathrm{k}_{0} ; 0 ; \mathrm{U}_{0}\right)$ and, in the soliton limit, as ${ }_{s}$ the image of by ( $c ; \quad) 7 !\left(0 ; @ M\left(c ; U_{s}\right) ; U_{s}\right)$.

Theorem 4. Under A ssumptions $7-2$ and with notation from Theorem 2 we have Harmonic limit Provided that $w_{0}$ does not vanish on , 
the averaged Hamiltonian $\mathrm{H}$ extends as a $\mathrm{C}^{2}$ function of $(\mathrm{k} ; ; \mathrm{M})$ to a connected open neighborhood (in ${ }^{8} R \quad\left(\operatorname{sign}\left(w_{0}\right) R_{+}\right) \quad R$ ) of 0 .

Soliton limit Provided that, for any $(c ;)^{2} 2, \varrho_{c}^{2} M\left(c ; U_{s}(c ;)\right) \in 0$, the averaged Hamiltonian $\mathrm{H}$ extends as a $\mathrm{C}^{2}$ function of $(\mathrm{k} ; \mathrm{M})$ to a connected open neighborhood (in $R_{+} R \quad R$ ) of $s$.

Proof. The proof is similar to the one of Theorem $3 \square$ In particular the issue is readily reduced to checking that the assumptions of Theorem 4 ensure that in the rel evant regimes $r_{; c ;}^{2}$ is invertible and $r_{k_{i ;}}^{2} \mathrm{M}$ possesses a limit, the study of the latter relying on (29).

In the harmonic limit, we have al ready checked all the required claims al ong the proof of Theorem 3 since there we have checked that $r_{i}^{2}$; possesses an invertible limit.

The soliton limit requires slightly more work. We already know from the proof of Theorem $B$ that both
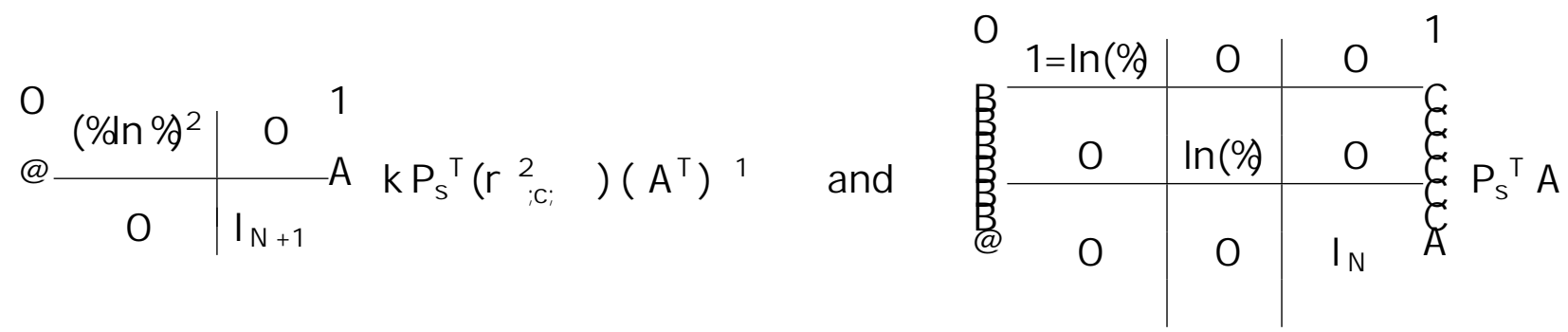

possess invertible limits. Thus the result stems from

$$
\begin{aligned}
& \mathrm{r}_{\mathrm{k} ; i}^{2} \mathrm{M} H+\mathrm{CB}_{0}^{1}
\end{aligned}
$$

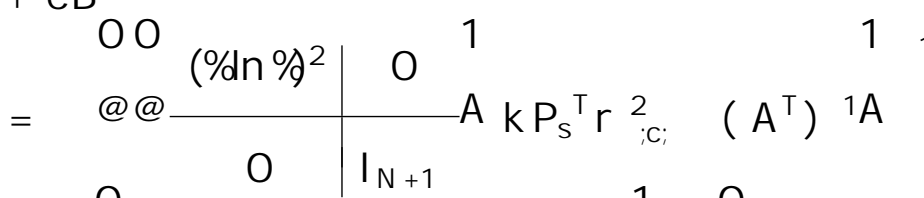

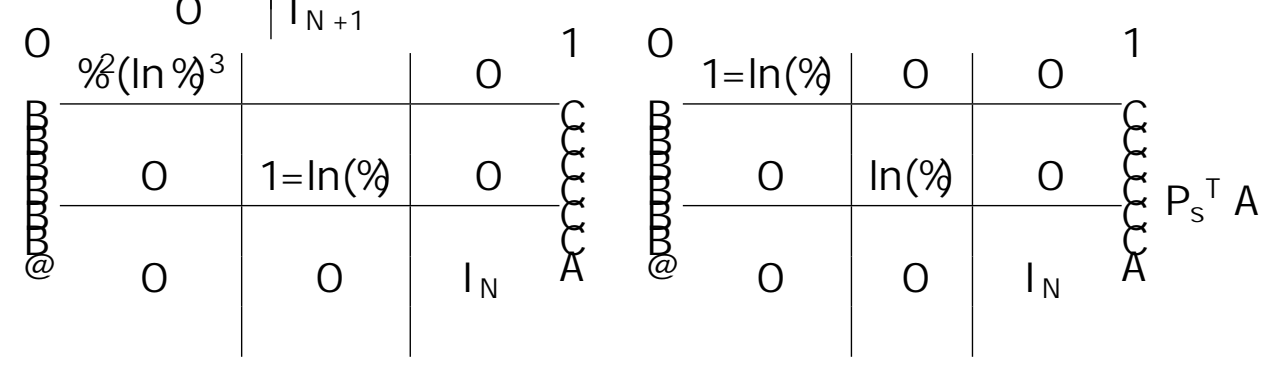

derived from (29).

\subsection{Basic features of the limiting modulated systems}

A detailed inspection of the proof of Theorem 4 provides explicit formulas for limiting values of $\mathrm{r}_{\mathrm{k} ; ;}^{2} \mathrm{M}$ thus of the Whitham matrix $W=B \mathrm{~K}_{\mathrm{k} ; ;}^{2} \mathrm{M} H$ in terms of coecients from Theorem 2. Yet rst we restrain from giving these and focus instead on what can be derived from more elementary arguments, using only the conclusion from Theorem 4,

\footnotetext{
${ }^{8} \mathrm{~N}$ ote that since is connected, $\mathrm{w}_{0}$ has a denite sign on 0 .
} 
that is, $\mathrm{C}^{2}$ regularity of $\mathrm{H}$. A long the discussion we shall still denote as $\mathrm{H}$ the extension of $\mathrm{H}$ to either 0 or $\mathrm{s}$.

To do so, we rst point out the elementary

$$
\text { Q } \mathrm{H}=\mathrm{C}=\mathrm{0} \text { on } 0 ; \quad \text { @ } \mathrm{H}=\mathrm{kc}=0 \text { on } \mathrm{s} ;
$$

which by dierentiating tangentially yield

$$
@^{2} \mathrm{H}=0 \text { and } @ r_{M} H=0 \text { on } 0 ; \quad @^{2} H=0 \text { and } @ r_{M} H=0 \text { on } s:
$$

In particular,

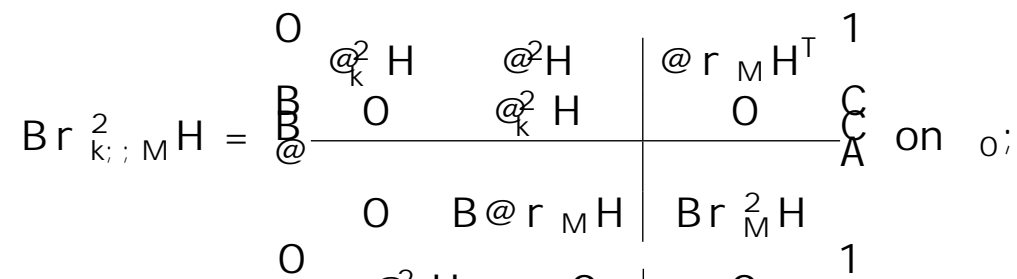

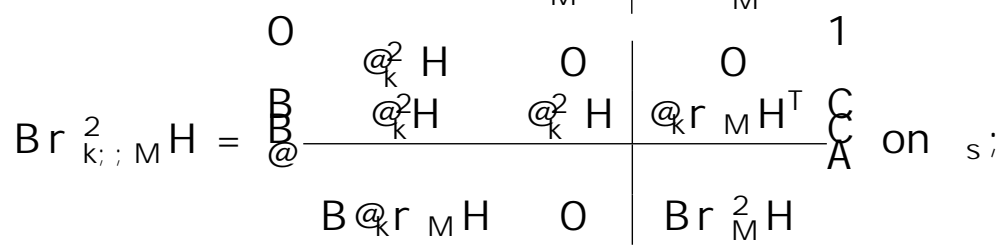

and a direct computation of a characteristic polynomial shows that, on either 0 or $s$, the spectrum of $W$ is the union, with multiplicity, of the spectrum of $\mathrm{Br}_{\mathrm{M}}^{2} \mathrm{H}$ and twice @ $^{2} \mathrm{H}$.

The fact that some second order derivatives of $\mathrm{H}$ are easier to compute is no accident. Since it is easy to extend $\mathrm{H}$ as a $\mathrm{C}^{1}$ map it is also straightforward to extend its second-order derivatives that contain at most one normal derivative. The hard parts of Theorem 4 are $\square$ the extensions of $@^{2} \mathrm{H}$ to 0 and, even more, of $@^{2} \mathrm{H}$ to s. To illustrate this further let us stress that for i $2 \mathrm{f} 0$; sg

$$
H\left(k_{;} ; U_{i}\right)=H\left(U_{i} ; 0\right) \text { on } i \text {; thus } r_{M}^{2} H\left(k ; \quad U_{i}\right)=r \underset{U}{2} H\left(U_{i} ; 0\right) \text { on i }
$$

so that, on i, $\mathrm{Br}_{\mathrm{M}}^{2} \mathrm{H}\left(\mathrm{k}_{;} ; \mathrm{U}_{\mathrm{i}}\right)$ is the characteristic matrix at $\mathrm{U}_{i}$ of the dispersionless system (ק). Likewise, for any $\left(\mathrm{k}_{0} ; \mathrm{U}_{0}\right)$ such that $\left(\mathrm{k}_{0} ; 0 ; \mathrm{U}_{0}\right) 20$

$$
\begin{aligned}
@_{\mathrm{Q}}^{2} \mathrm{H}\left(\mathrm{k}_{0} ; 0 ; \mathrm{U}_{0}\right) & =\bigotimes_{(}\left(\mathrm{k} \mathrm{c}_{0}\right)\left(\mathrm{k}_{0} ; \mathrm{U}_{0}\right)=\mathrm{v}_{\mathrm{g}}\left(\mathrm{k}_{0} ; \mathrm{U}_{0}\right) ; \\
@ \mathrm{r}_{\mathrm{M}} \mathrm{H}\left(\mathrm{k}_{0} ; 0 ; \mathrm{U}_{0}\right) & =\mathrm{k}_{0} \mathrm{r}_{\mathrm{U}} \mathrm{C}_{0}\left(\mathrm{k}_{0} ; \mathrm{U}_{0}\right) ;
\end{aligned}
$$

where $v_{g}\left(k_{0} ; U_{0}\right)$ is the linear group velocity of the harmonic wavetrain on $U_{0}$ at wavelength $k_{0}$. Similarly, at the soliton limit, we have for any $\left(C_{s} ; U_{s}\right) 2$

$$
@ H\left(0 ; @ M\left(c_{s} ; U_{s}\right) ; U_{s}\right)=M\left(c_{s} ; U_{s}\right) \quad c_{s} @ M\left(c_{s} ; U_{s}\right)
$$

so that for any $\left(c_{s} ; U_{s}\right) 2$

$$
\begin{aligned}
& \oint^{2} H\left(0 ; @ M\left(c_{s} ; U_{s}\right) ; U_{s}\right)=c_{s} ; \\
& \text { @r } r_{M} H\left(0 ; @ M\left(c_{s} ; U_{s}\right) ; U_{s}\right)=r_{U} M\left(c_{s} ; U_{s}\right) \text { : }
\end{aligned}
$$

Going back to the cancellations in $\mathrm{Br}_{\mathrm{k} ; ;}^{2} \mathrm{M}$, we make the following elementary algebraic observation, whose proof follows from a short computation | left to the reader. 
Lemma 1. For any real numbers $\mathrm{V}$ and $\mathrm{a}$, any vectors $\mathrm{I}_{0}, \mathrm{r}_{0}$ in $\mathrm{R}^{\mathrm{N}}$ and any $\mathrm{N} \quad \mathrm{N}$ matrix $M$ such that $v$ is not an eigenvalue of $M$ we have
\begin{tabular}{lll|ll}
0 & 1 & 0 & $I^{\top}$ & 1 \\
B & 0 & 1 & 0 & $\AA$ \\
\hline 0 & & $I_{N}$ & \\
0 & 1 & 0 & 0 & 1 \\
B & 0 & 1 & $I^{\top}$ & $\AA$ \\
\hline$r$ & 0 & $I_{N}$ &
\end{tabular}

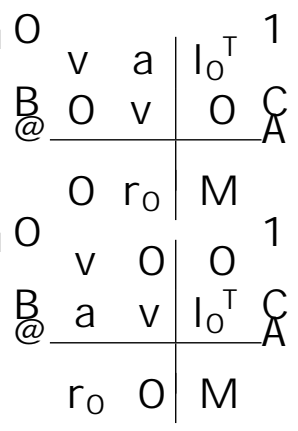
10
\begin{tabular}{ll|l|l|l}
1 & 0 & $I^{\top}$ \\
\hline
\end{tabular}
$\AA=\stackrel{B}{\varrho}$
\begin{tabular}{llllll|l}
0 & $v$ & $a$ & $I_{0}^{\top}\left(M \quad v I_{N}\right)^{1} r_{0}$ & 0 & 1 \\
0 & 0 & & 0 & $\AA$ \\
\hline 0 & 0 & & $M$
\end{tabular}

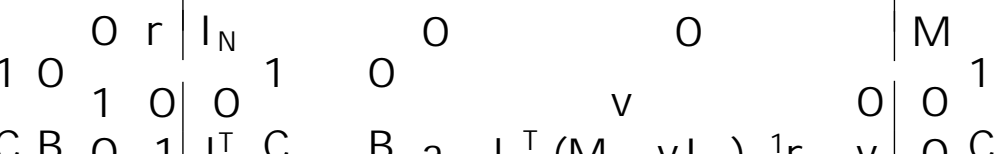

with

$$
I:=\left(\begin{array}{lll}
M^{\top} & v I_{N}
\end{array}\right)^{1} I_{0} ; \quad r:=\left(\begin{array}{lll}
M & v I_{N}
\end{array}\right)^{1} r_{0}:
$$

Note that the two matrices considered in Lemma 1 are obtained one from the other merely by exchanging the rst and second coordinates. We have introduced these two cases just to emphasize that this al gebraic lemma applies to both kinds of limit.

For convenience, let us summarize part of the foregoing ndings in the following statement.

Corollary 5. Under the assumptions of Theorem 4 4 till denoting by $\mathrm{H}$ its extension to either or s, we have

Harmonic limit At any point $\left(\mathrm{k}_{0} ; 0 ; \mathrm{U}_{0}\right)$ of 0 , the spectrum of the characteristic matrix of the modulation system $\mathrm{B}{ }_{\mathrm{k} ; ;}^{2} \mathrm{M}\left(\mathrm{k}_{0} ; 0 ; \mathrm{U}_{0}\right)$ is given, with al gebraic multiplicity, by the spectrum of the dispersionless characteristic matrix $\mathrm{B} \mathrm{r}_{\mathrm{U}}^{2} \mathrm{H}\left(\mathrm{U}_{0} ; 0\right)$ and twice the linear group velocity $\mathrm{v}_{\mathrm{g}}\left(\mathrm{k}_{0} ; \mathrm{U}_{0}\right)=\oint^{2} \mathrm{H}\left(\mathrm{k}_{0} ; 0 ; \mathrm{U}_{0}\right)$, so that in particular the modulation system is weakly hyperbolic if and only if the dispersionless system is so.

Moreover, $\mathrm{V}_{\mathrm{g}}\left(\mathrm{K}_{0} ; \mathrm{U}_{0}\right)$ is a semisimple characteristic of (22) if and only if @2 $\mathrm{H}\left(\mathrm{k}_{0} ; 0 ; \mathrm{U}_{0}\right)$ coincides with 9

$$
\begin{aligned}
\left(@ r_{M} H\right)^{\top}\left(r_{M}^{2} H\right. & \left.\left(@^{2} H\right) B{ }^{1}\right)^{1} @ r_{M} H \\
= & k_{0}^{2}\left(r \cup C_{0}\right)^{\top}\left(r_{U}^{2} H\left(U_{0} ; 0\right)+v_{g} B{ }^{1}\right){ }^{1} r_{U} C_{0}
\end{aligned}
$$

so that the modulation system is hyperbolic if and only if the foregoing condition is satised and the dispersionless system is hyperbolic.

Soliton limit For any $\left(C_{s} ; U_{s}\right)$ of , the spectrum of the characteristic matrix of the modulation system $B r_{k ; i}^{2} \mathrm{M}\left(0 ; @ M\left(c_{s} ; U_{s}\right) ; U_{s}\right)$ is given, with algebraic multiplicity, by the spectrum of the dispersionless characteristic matrix $\quad B \quad r{ }_{U}^{2} \mathrm{H}\left(\mathrm{U}_{s} ; 0\right)$ and twice the soliton velocity $c_{s}=@^{2} H\left(0 ; @ M\left(c_{s} ; U_{s}\right) ; U_{s}\right)$, so that in particular the modulation system is always weakly hyperbolic.

M oreover, $c_{s}$ is a semisimple characteristic of (22) if and only if $@^{2} \mathrm{H}\left(0 ; @ M\left(\mathrm{c}_{s} ; \mathrm{U}_{s}\right) ; \mathrm{U}_{s}\right)$

\footnotetext{
${ }^{9}$ The left-hand side being evaluated at $\left(\mathrm{k}_{0} ; 0 ; \mathrm{U}_{0}\right)$ and the right-hand side at $\left(\mathrm{k}_{0} ; \mathrm{U}_{0}\right)$.
} 
coincides with 10

$$
\begin{aligned}
\left(@ r_{M} H\right)^{\top}\left(r_{M}^{2} H\right. & \left.\left(@^{2} H\right) B{ }^{1}\right){ }^{1} @ r_{M} H \\
= & \left(r_{U} M\right)^{\top}\left(r_{U}^{2} H\left(U_{s} ; 0\right)+c_{s} B{ }^{1}\right){ }^{1} r_{U} M
\end{aligned}
$$

so that the modulation system is hyperbolic if and only if the foregoing condition is satised.

Proof. At the soliton limit, the only thing left is to check that from the assumptions of Theorem 4 stem that $\mathrm{C}_{s}$ is not an eigenvalue of $\mathrm{B} \mathrm{r} \underset{U}{2} \mathrm{H}\left(\mathrm{U}_{s} ; 0\right)$ and that eigenvalues of the latter matrix are real and distinct. Yet a relatively direct computation (for which the reader is referred to A ppendix $A$ and [BGMRar, A ppendix A]) shows that

$$
\operatorname{det}\left(B r{ }_{U}^{2} H\left(U_{s} ; 0\right)+C_{s} I_{N}\right)=\begin{array}{ll}
b @ W\left(v_{s} ; c_{s} ; s\right) & \text { if } \quad N=1 \\
b^{2}\left(v_{s}\right) @ W\left(v_{s} ; c_{s} ; s\right) & \text { if } \quad N=2
\end{array}
$$

so that the conditions stem from A ssumption 2 that contains $@_{s}^{2} W\left(v_{s} ; c_{s} ; s\right)<0$.

At the harmonic limit, we only need to check that from the assumptions of Theorem 4 stems that $\mathrm{V}_{\mathrm{g}}$ is not an eigenvalue of $\mathrm{B} \mathrm{r} \mathrm{U}_{\mathrm{U}}^{2} \mathrm{H}\left(\mathrm{U}_{0} ; 0\right)$. We rst stress that the relation pointed out above also holds for $U_{0}$ (instead of $U_{s}$ ) so that

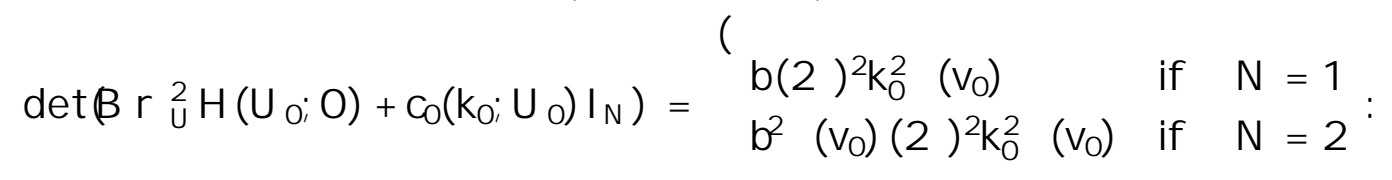

Multiplying rst the latter by $k_{0}$ then dierentiating it with respect to $k_{0}$ yield by the $\mathrm{N}$-linearity of the determinant

$$
\begin{aligned}
& \operatorname{det}\left(B r_{(}{ }_{U} H\left(U_{0} ; 0\right)+v_{g}\left(k_{0} ; U_{0}\right) I_{N}\right) \\
&=\begin{array}{llll}
3 b(2)^{2} k_{0}^{2} & \left(v_{0}\right) & \text { if } & N=1 \\
3 b^{2}\left(v_{0}\right)(2 \quad)^{2} k_{0}^{2} & \left(V_{0}\right)+\left(k_{0} @ c_{0}\left(k_{0} ; U_{0}\right)\right)^{2} & \text { if } & N=2
\end{array}:
\end{aligned}
$$

This proves the claim.

Remark 12. Concerning the case $\mathrm{N}=2$, at the harmonic limit, note that $\mathrm{B} r \underset{\mathrm{U}}{2} \mathrm{H}\left(\mathrm{U}_{0} ; 0\right)$ is never diagonal and that

$$
\left.\left.\left(\operatorname{tr}\left(B r{ }_{U}^{2} H\left(U_{0} ; 0\right)\right)\right)^{2} \quad 4 \operatorname{det}\left(B r{ }_{U}^{2} H\left(U_{0} ; 0\right)\right)=4 b^{2}\left(v_{0}\right) \quad f 0 q v_{0}\right)+\frac{1}{2} 0 q v_{0}\right) u_{0}^{2}
$$

so that the dispersionless system is weakly hyperbolic (resp. hyperbolic) at $U_{0}$ if and only if $\left.\left.f \circ q v_{0}\right)+\frac{1}{2} \quad 0 q v_{0}\right) u_{0}^{2} \quad 0\left(\right.$ resp. $\left.\left.\left.f o q v_{0}\right)+\frac{1}{2} \quad 0 q v_{0}\right) u_{0}^{2}>0\right)$. In particular when is ane, as is the case for Euler-K orteweg systems, this condition reduces to the requirement that $f$ be convex, which is the usual hyperbolicity condition for the Euler systems in terms of pressure monotonicity.

\footnotetext{
${ }^{10}$ The left-hand side being evaluated at $\left(0 ; @ M\left(c_{s} ; U_{s}\right) ; U_{s}\right)$ and the right-hand side at $\left(c_{s} ; U_{s}\right)$.
} 
Despite the symmetry of Corollary 5 with respect to permutation of the variables $k$ and , the harmonic and soliton limits dier signicantly in terms of the hyperbolic nature of the limiting system. Indeed as it follows from the analysis expounded in next subsection, the condition of Corollary 5 ensuring the semisimplicity of the characteristic value $@^{2} \mathrm{H}$ is always satised at the soliton limit whereas in general it fails at the harmonic limit. In particular as we show in A ppendix $A$ the latter condition does fail for the classical K orteweg \{de Vries equation.

Note however that the direct consequences of this discrepancy on the original modulation systems (and not their limiting extensions) are almost immaterial. Indeed whereas the failure of weak hyperbolicity (as potentially caused here by the failure of weak hyperbolicity of the dispersionless system) is stable under perturbation, neither hyperbolicity nor failure of hyperbolicity are stable phenomena in the presence of a multiple root. The determination of the nature of the original modulation systems will require an even ner analysis than the one carried out in next subsection.

\subsection{Explicit formulas for the limiting modulation systems}

Now, to push our analysis a bit further, we extract from the proof of Theorem 4 explicit formulas for the limiting values of $\mathrm{r}_{\mathrm{k} ; i}^{2} \mathrm{M}$, in particular for $@^{2} \mathrm{H}$ in the harmonic limit and for $@^{2} \mathrm{H}$ in the soliton limit.

Let us begin with the harmonic limit. For concision's sake we rst introduce

$$
=\begin{array}{lllllll}
8 & & & & & \text { if } N=1 \\
3 & 2 c_{0} b^{2} & & & \\
3 & 2 c_{0} & 2 & 2 c_{0} & 0 & W_{0} & \\
3 & 2 c_{0} & 0 & W_{0} & 2 c_{0} W_{0}^{2} & 2 & \text { if } N=2
\end{array}
$$

and

$$
x_{0}=\begin{array}{ccc}
8 & b_{0} b^{1} \\
3 & b_{0} 0 & \text { if } N=1 \\
3 & b_{0} w_{0}+c_{0} & 0
\end{array}
$$

so that it follows from Theorem 2 and Corollary 3 that

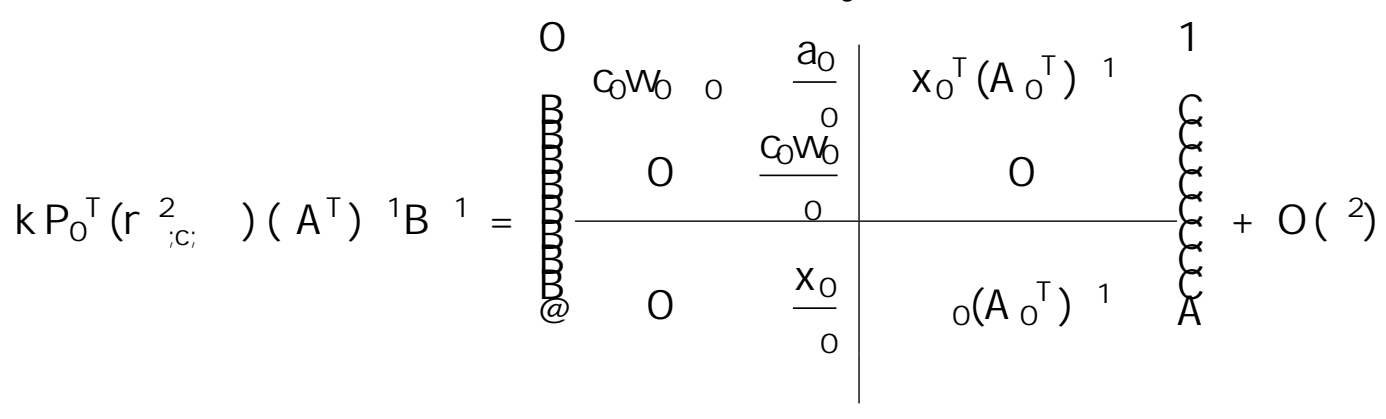


thus its inverse is

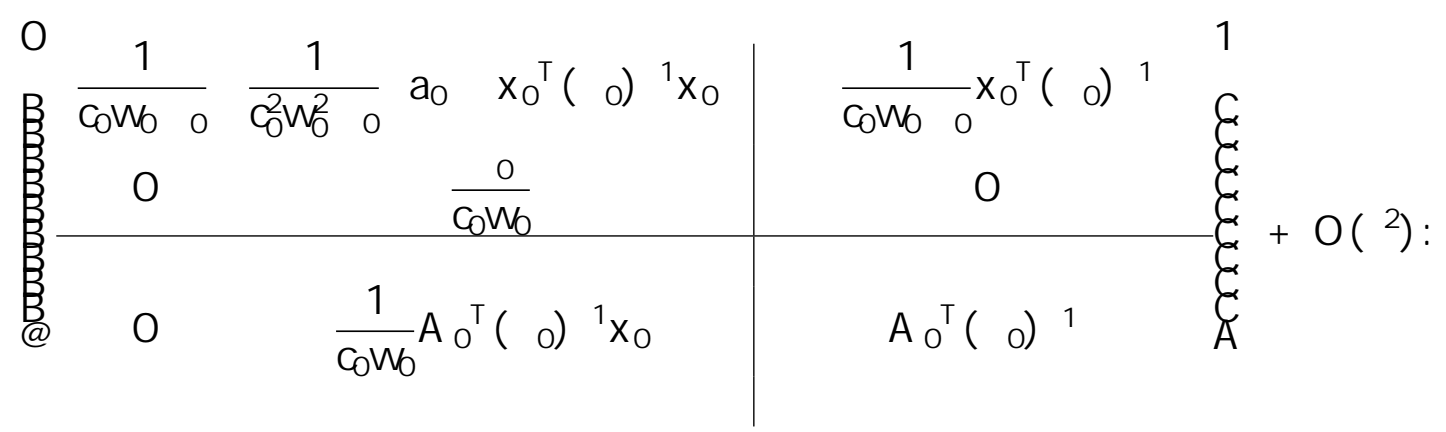

From this stems that $\frac{1}{\mathrm{k}} B A^{\top}\left(r_{;}^{2} ; ;\right) \quad{ }^{1} A$ equals

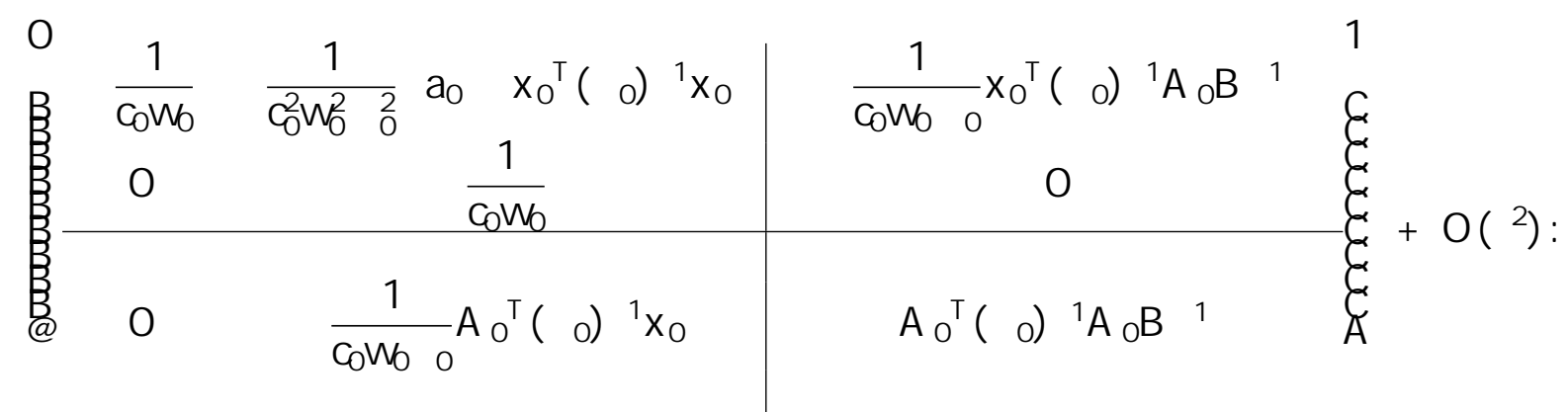

In particular, on $\quad 0$,
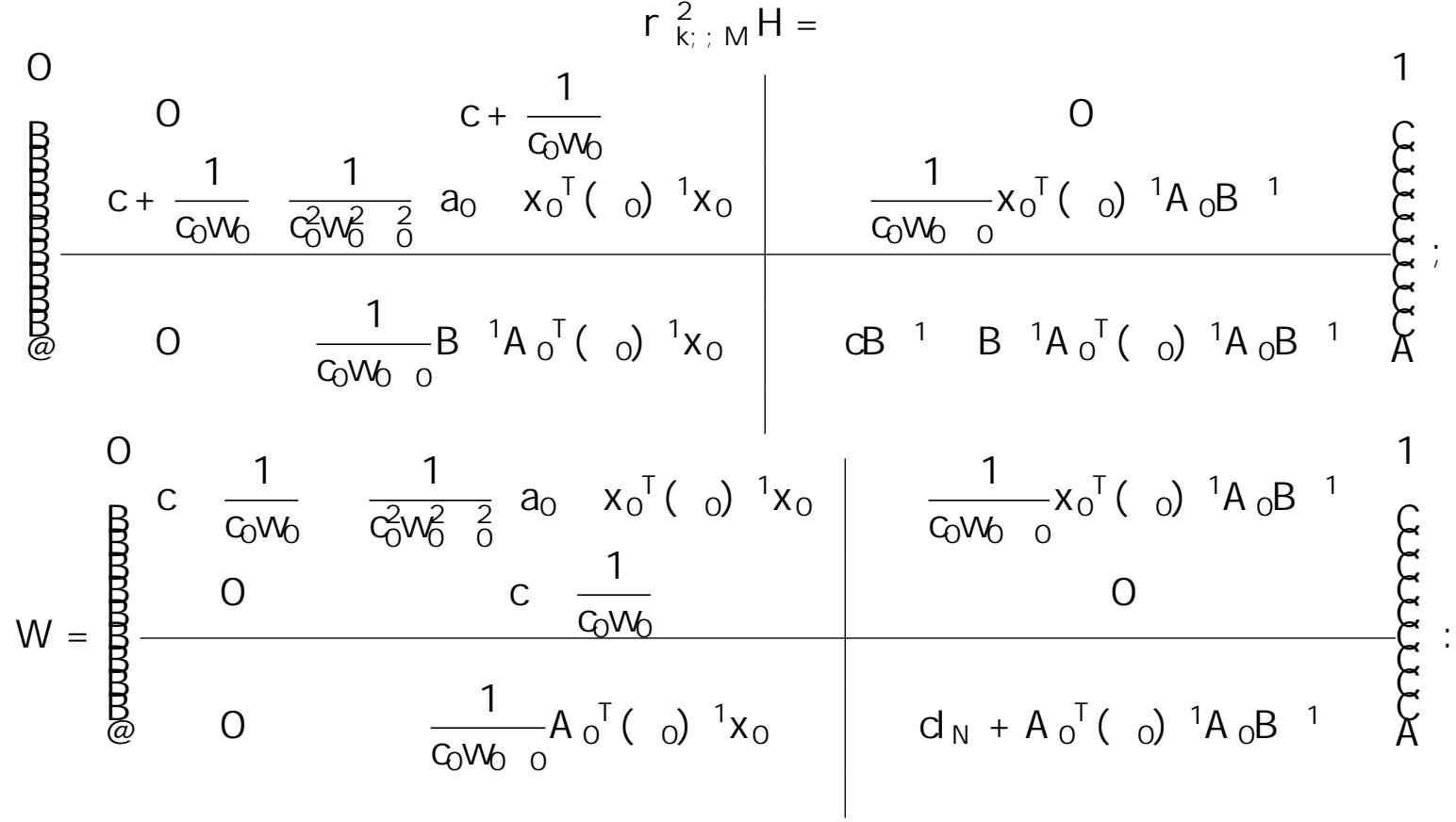

Translating, by identication, the foregoing computations into the notation of Corollary 5 yields the following result. 
Theorem 5. Under the assumptions of Theorem 4 Det us still denote by $\mathrm{H}$ and $\mathrm{W}$ their extensions to 0 . Then at any point $\left(\mathrm{k}_{0} ; 0 ; \mathrm{U}_{0}\right)$ of 0 , we have $\mathrm{E}^{\mathrm{TI}}$

$$
\begin{aligned}
& @^{2} H=k_{0}^{4}\left(@ C_{0}\right)^{2} a_{0}+k_{0}^{2} r \cup C_{0}^{\top}\left(r \underset{u}{2} H(U, 0)+C_{0} B{ }^{1}\right){ }_{1}^{1} r_{U} \cup C_{0}
\end{aligned}
$$

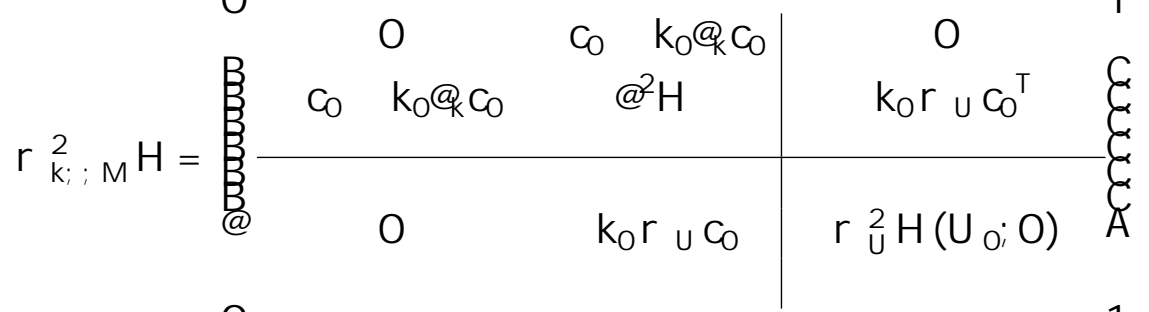

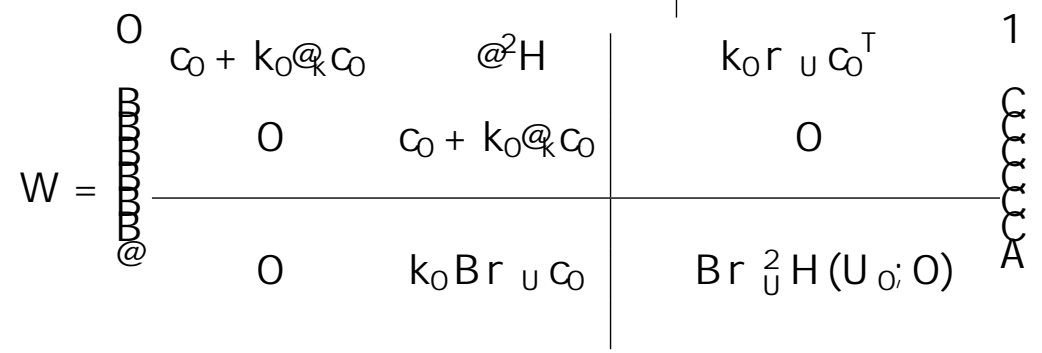

where $\mathrm{a}_{0}$ is as in Theorem $\square$

$$
a_{0}=k_{0} @ \varrho^{2}\left(0 ; c_{0} ; 0\right):
$$

In particular, at any $\left(\mathrm{k}_{0} ; 0 ; \mathrm{U}_{0}\right)$ of $0_{0}^{0}$, denoting $\mathrm{v}_{\mathrm{g}}=\mathrm{c}_{0}+\mathrm{k}_{0} @ \mathrm{c}_{0}\left(\mathrm{k}_{0} ; \mathrm{U}_{0}\right)$, we have

$$
\beta_{0}{ }^{1} W \beta_{0}=@ \begin{array}{cc|c}
v_{g} & a_{0} & 0 \\
0 & v_{g} & 0 \\
\hline 0 & 0 & B r U_{U}^{2} H\left(U_{0} ; 0\right)
\end{array}
$$

with

$$
\begin{aligned}
& \mathrm{a}_{0}=\mathrm{k}_{0}^{4}\left(@ \mathrm{c}_{0}\right)^{2} \mathrm{a}_{0} \quad \mathrm{k}_{0}^{2} \mathrm{r} \cup \mathrm{c}_{0}^{\top}\left(\mathrm{r}_{\mathrm{U}}^{2} \mathrm{H}\left(\mathrm{U}_{0} ; 0\right)+\mathrm{c}_{0} B{ }^{1}\right){ }^{1} \mathrm{r} \cup \mathrm{c}_{0} \\
& +k_{0}^{2} r \cup C_{0}^{\top}\left(r_{U}^{2} H\left(U_{0} ; 0\right)+v_{g} B{ }^{1}\right){ }^{1} r_{\cup} \cup C_{0}
\end{aligned}
$$

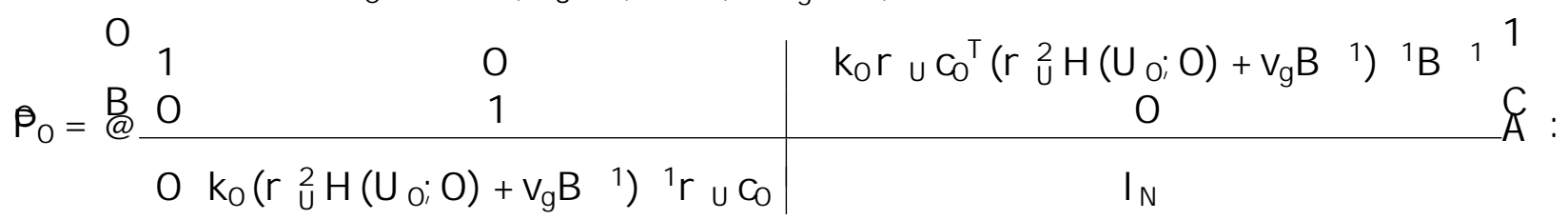

As already announced, in general $a_{0}$ is not zero and $W$ possesses a Jordan block associated with $v_{g}$. In particular, in A ppendix $A$ we check that no vanishing occurs for the classical KdV equation.

Now we turn to the solitgn limit. For concision's sake we rst introduce

$$
s=\begin{array}{cccccc}
2 c_{s} b^{2} & & \text { if } N=1 \\
3 & 2 c_{s} & 2 & 2 c_{s} s_{s} w_{0} & \text { if } N=2 \\
2 c_{s} & s & W_{0} & 2 c_{s} w_{s}^{2}+s_{s}^{2} &
\end{array}
$$

\footnotetext{
${ }^{11}$ The left-hand side being evaluated at $\left(\mathrm{k}_{0} ; 0 ; \mathrm{U}_{0}\right)$ and the right-hand side at $\left(\mathrm{k}_{0} ; \mathrm{U}_{0}\right)$.
} 
and

$$
\begin{aligned}
& \sum^{8}\left(S^{1} W_{s}\right) O_{s} S^{1} V_{s} \text { ! if } N=1 \\
& \mathrm{y}_{\mathrm{s}}=3 \begin{array}{lll}
\left(\mathrm{S}^{1} \mathrm{~T}_{\mathrm{s}}\right) & \mathrm{O}_{\mathrm{s}} \mathrm{S}^{1} \mathrm{~V}_{\mathrm{s}} \\
\left(\mathrm{S}^{1} \mathrm{~W}_{\mathrm{s}}\right) & \mathrm{O}_{\mathrm{s}} \mathrm{S}^{1} \mathrm{~V}_{\mathrm{s}}
\end{array} \quad \text { if } \mathrm{N}=2
\end{aligned}
$$

so that it follows from Theorem 2 and Corollary 3 that

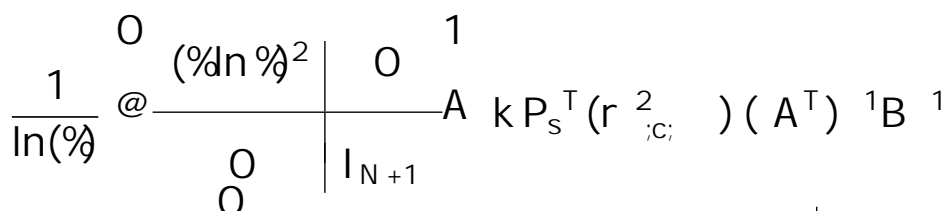

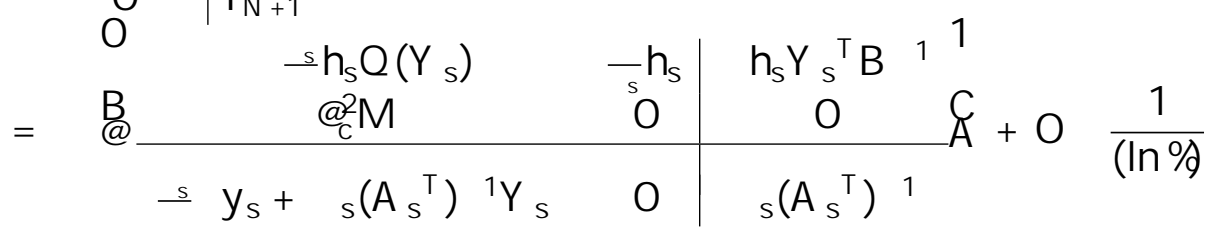

and its inverse is

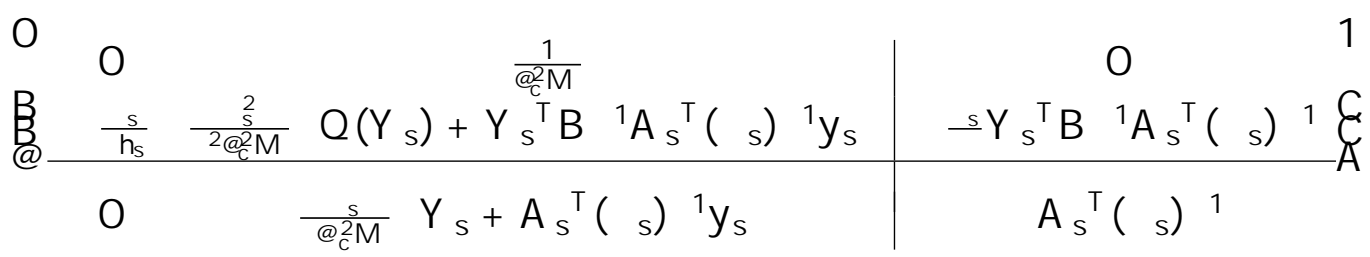

$$
\begin{aligned}
& +0 \frac{1}{(\ln \%)} \text { : }
\end{aligned}
$$

From this stems that $\frac{1}{\mathrm{k}} B A^{\top}\left(r_{; c_{;}}^{2}\right){ }^{1} A$ equals

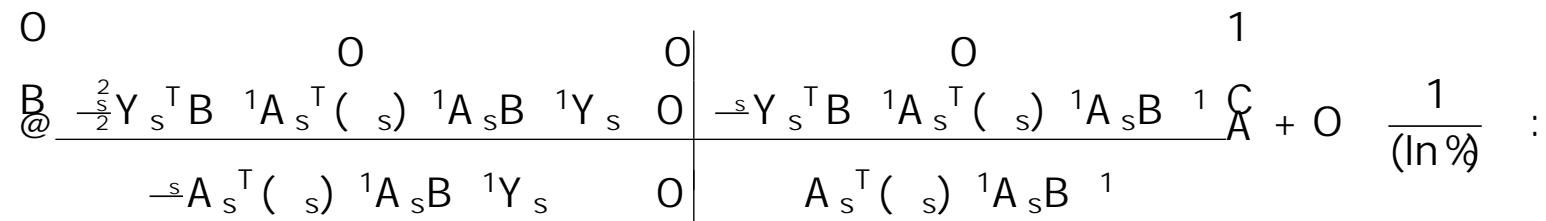

In particular, on s,

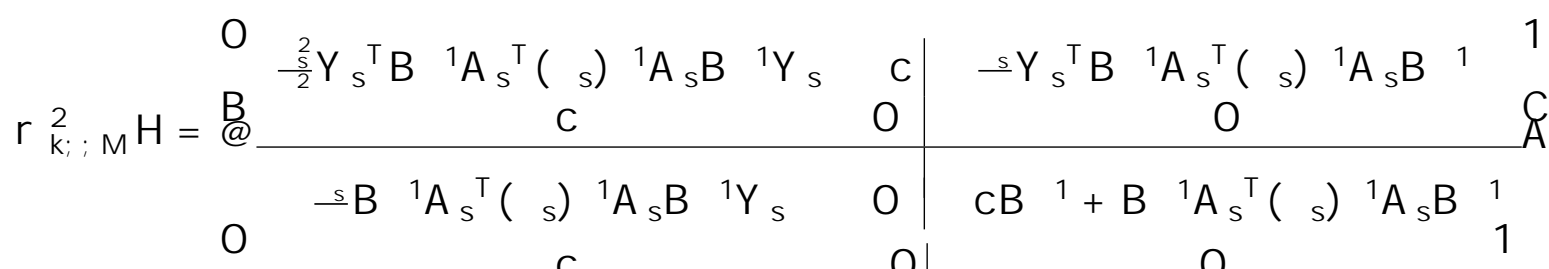

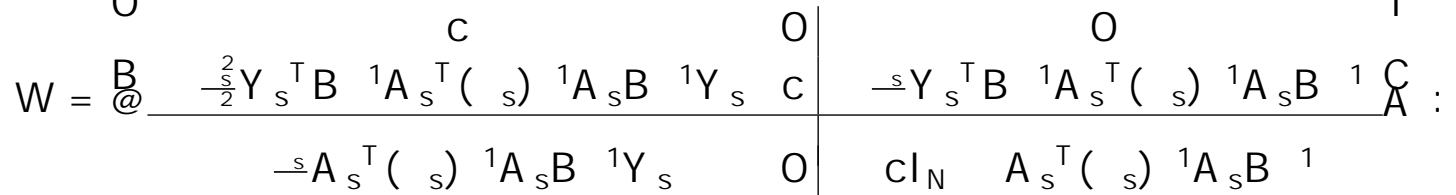

Translating again, by identication, into the notation of Corollary 5 yie/ds the following result. 
Theorem 6. Under the assumptions of Theorem 4 Det us still denote by $H$ and $W$ their extensions to $s$. Then for any $\left(c_{s} ; U_{s}\right)$ of , we have $e^{12}$

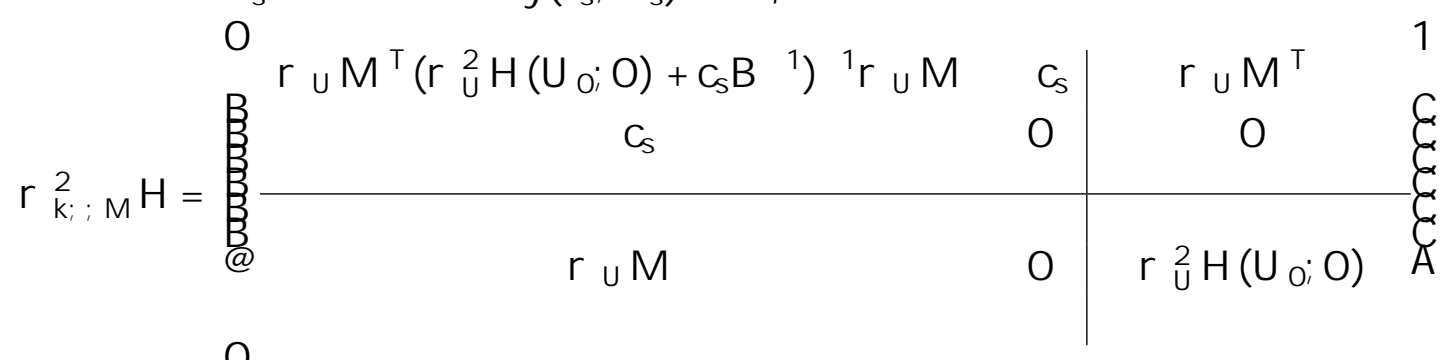

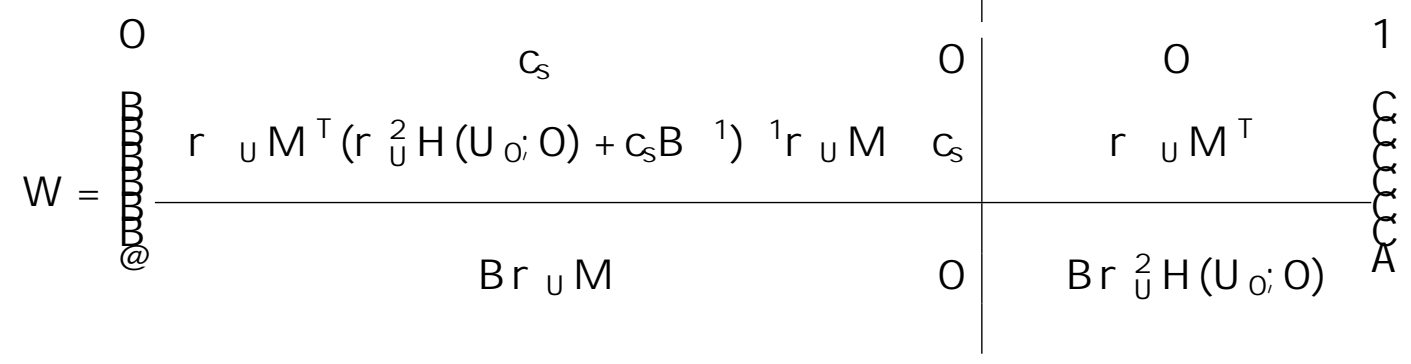

thus

$$
\beta_{S}{ }^{1} W \beta_{S}=\begin{array}{c|c}
C_{s} I_{2} & 0 \\
\hline 0 & B r \underset{U}{2} H\left(U_{0} ; 0\right)
\end{array}
$$

with

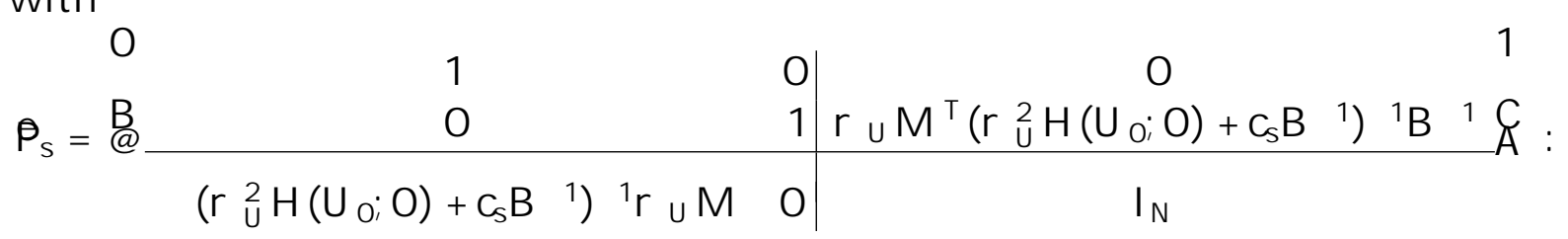

Remark 13. In both limits, the $2 \quad 2$ principal block of $\mathrm{r}^{2} \mathrm{H}$ has negative determinant hence signature $(1 ; 1)$, and therefore $r^{2} \mathrm{H}$ is neither positive denite nor negative denite in either regime.

\section{A symptotics of the modulation eigenelds}

Since limiting characteristic matrices exhibit double roots, we need to perform a higherorder asymptotic analysis so as to determine the hyperbolic nature of modulation systems not at the limit of interest but near the distinguished limit. We undertake this task now.

\subsection{Small amplitude regime}

In the harmonic regime, the $\mathrm{N}$ eigenvalues arising from those of $\mathrm{Br}{ }_{U}^{2} \mathrm{H}\left(\mathrm{U}_{0} ; 0\right)$ may be analyzed by standard spectral perturbation analysis. We only need to blow up the two eigenvalues near $v_{g}$ and we shall do it by inverting and scaling $W \quad v_{g} I_{N+2}$ so as to reduce the problem to the spectral perturbation of simple eigenvalues.

\footnotetext{
${ }^{12}$ The left-hand side being evaluated at $\left(0 ; @ M\left(c_{s} ; U_{s}\right) ; U_{s}\right)$ and the right-hand side at $\left(C_{s} ; U_{s}\right)$.
} 
The scaling process will reveal the prominent role played by some of the higher-order correctors not made explicit in Theorem 2. $\square \mathrm{W}$ ith this in mind, note that the proof of Theorem 2, in [BGMRarl] also gives that under the assumptions of Theorem 2, $\square \mathrm{r}{ }_{; ; \text {; }}^{3}$ possesses a limit with convergence rate $O()$ when ! 0 . This implies that possesses as a function of $(; C ; \quad)$ a $C^{3}$ extension to the limit $=0$ with convergence rate $O(\mathrm{l}$. In turn this implies, under the assumptions of Theorem 3, that $\mathrm{H}$ as a function of $(\mathrm{k} ;$; $\mathrm{M})$ possesses a $\mathrm{C}^{3}$ extension to 0 , with convergence rate $\mathrm{O}(\mathrm{)})$. Then proceeding as in Subsection 4.2, we deduce that

$$
\begin{aligned}
@_{k}^{2} H\left(k_{0} ; ; U_{0}\right) & =@_{k}^{3} H\left(k_{0} ; 0 ; U_{0}\right)+O\left({ }^{3}\right) ; \\
@ r_{M} H\left(k_{0} ; ; \quad U_{0}\right) & =@_{k}^{2} r_{M} H\left(k_{0} ; 0 ; U_{0}\right)+O\left({ }^{3}\right) ;
\end{aligned}
$$

with

$$
\begin{aligned}
& \bigotimes_{k}^{3} H\left(k_{0} ; 0 ; U_{0}\right)=@^{2}\left(k c_{0}\right)\left(k_{0} ; U_{0}\right)=2 @ c_{0}\left(k_{0} ; U_{0}\right) k_{0} @^{2} c_{0}\left(k_{0} ; U_{0}\right) ; \\
& @^{2} r_{M} H\left(k_{0} ; 0 ; U_{0}\right)=@ r_{U}\left(k c_{0}\right)\left(k_{0} ; U_{0}\right)=r \quad \cup C_{0}\left(k_{0} ; U_{0}\right) \quad k_{0} @ r_{U} C_{0}\left(k_{0} ; U_{0}\right):
\end{aligned}
$$

As a consequence, with notation from Theorem 5, we have

$$
\begin{aligned}
& \beta_{0}{ }^{1} \mathrm{~W}\left(\mathrm{k}_{0} ; \quad \mathrm{U}_{0}\right) \mathrm{\beta}_{0}=
\end{aligned}
$$

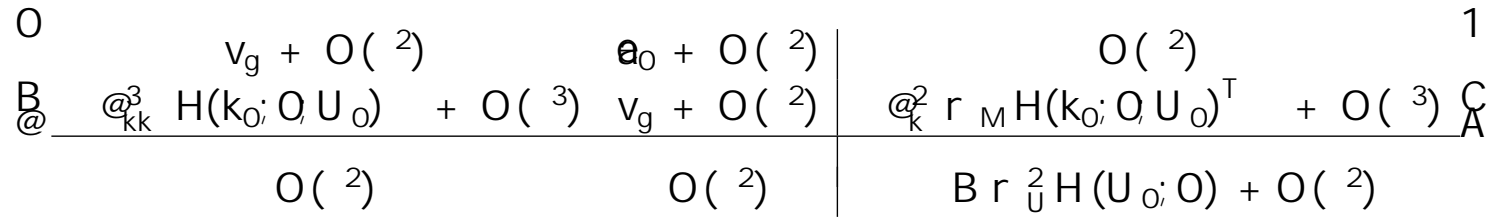

so that when $a_{0} @_{k}^{2} r_{M} H\left(k_{0} ; 0 ; U_{0}\right) \in 0$

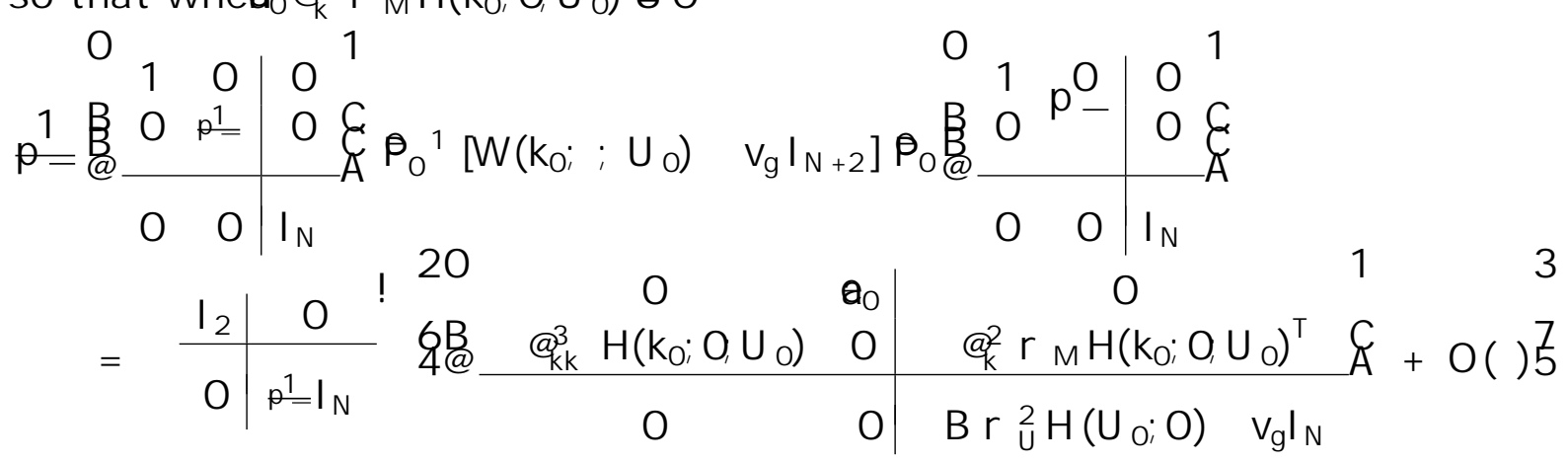

is invertible (provided that is suciently small) and its inverse is

$$
\begin{array}{ccc|c}
0 & 0 & \frac{1}{1} & 0 \\
\beta & \Theta_{k k}^{3} \frac{H\left(k_{0} ; 0 ; U_{0}\right)}{a_{0}} & 0 & 0 \xi+O(): \\
\hline 0 & 0 & 0
\end{array}
$$

At last we may apply elementary spectral perturbation theory to the latter matrix to study its two simple eigenvalues near $\quad 1=\frac{a_{0} @_{k k}^{3} H\left(k_{0} ; 0 ; U_{0}\right)}{\text { (where here }}{ }^{P}$ - denotes any determination of the square root function). This leads to the following result. 
Theorem 7. Under the assumptions of Theorem 4 ,et us still denote by $\mathrm{H}$ its extension to 0 and consider $\left(\mathrm{k}_{0} ; 0 ; \mathrm{U}_{0}\right) 2$, with associated linear group velocity

$$
\mathrm{V}_{\mathrm{g}}\left(\mathrm{k}_{0} ; \mathrm{U}_{0}\right)=\mathrm{Q}^{2} \mathrm{H}\left(\mathrm{k}_{0} ; 0 ; \mathrm{U}_{0}\right)=\mathrm{c}_{0}\left(\mathrm{k}_{0} ; \mathrm{U}_{0}\right)+\mathrm{k}_{0} @ \mathrm{C}_{0}\left(\mathrm{k}_{0} ; \mathrm{U}_{0}\right):
$$

Then in the small amplitude regime, the spectrum of the Whitham matrix $W\left(k_{0} ; ; U_{0}\right)$ contains

1. two eigenvalues near $v_{g}$, that expand as

$$
v_{g} \quad P-\frac{M I}{M}+0()
$$

(where here ${ }^{\mathrm{p}}-$ denotes some determination of the square root function), with corresponding eigenvectors

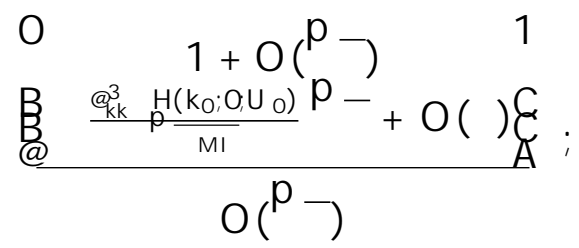

provided that the modulational-instability index $\quad \mathrm{MI}_{\mathrm{I}}\left(\mathrm{k}_{0} ; \mathrm{U}_{0}\right)$, given by ${ }^{13}$

$$
\begin{aligned}
& M I:=\quad k_{0}^{5}\left(@ C_{0}\right)^{2} @^{2}\left(\quad 0 ; C_{0} ; \quad 0\right) \quad k_{0}^{2} r \cup C_{0}^{\top}\left(r \underset{\cup}{2} H(U 0 ; 0)+C_{0} B{ }^{1}\right){ }^{1} r \cup C_{0} \\
& +k_{0}^{2} r \cup C_{0}^{\top}\left(r{ }_{\cup}^{2} H\left(U_{0} ; 0\right)+v_{g} B{ }^{1}\right){ }^{1} r \cup C_{0} \\
& 2 @ c_{0}+k_{0} @^{2} c_{0} \\
& =@^{2} H \quad\left(@ r_{M} H\right)^{\top}\left(r_{M}^{2} H \quad\left(@_{k}^{2} H\right) B{ }^{1}\right)^{1} @ r_{M} H \quad @_{k}^{3} H \text {; }
\end{aligned}
$$

is not zero ;

2. and $\mathrm{N}$ eigenvalues near the the eigenvalues of the dispersionless characteristic matrix $\mathrm{B} \mathrm{r} \underset{\mathrm{U}}{2} \mathrm{H}\left(\mathrm{U}_{0} ; 0\right)$, that expand as

$$
z_{j}+O() ; \quad j 2 f 1 ; N g
$$

with associated eigenvectors

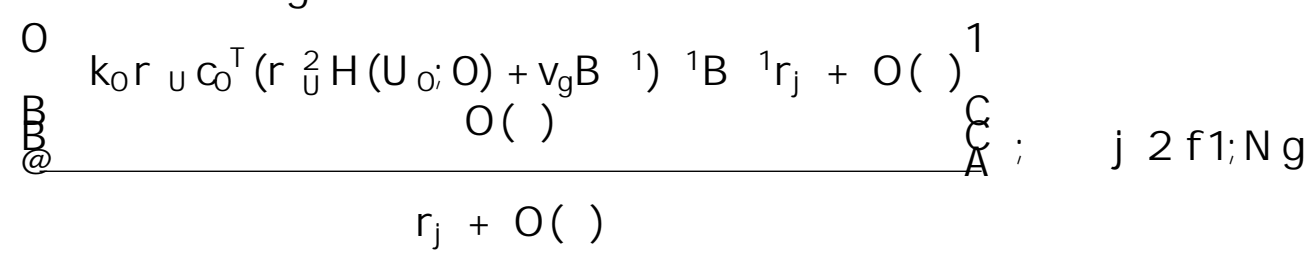

where $z_{j}, j 2 f 1 ; N g$, are the eigenvalues of $B{ }_{U}^{2} H\left(U_{0} ; 0\right)$, with corresponding eigenvectors, $r_{j}$, j $2 \mathrm{f} 1 ; \mathrm{Ng}$, provided that these $\mathrm{N}$ eigenvalues are distinct.

\footnotetext{
${ }^{13} \mathrm{~W}$ ith evaluation either at $\left(\mathrm{k}_{0} ; \mathrm{U}_{0}\right)$ or at $\left(\mathrm{k}_{0} ; 0 ; \mathrm{U}_{0}\right)$, depending on terms.
} 
Moreover all the bounds are locally uniform with respect to $\left(\mathrm{k}_{0} ; \mathrm{U}_{0}\right)$.

Note that the existence of an expansion into powers of $\quad P-$ of the eigenvalues of an $O($ ) perturbation of a matrix possessing a double root from which they emerge is consistent with the general | worst-case | algebraicity theory for the spectrum of matrices.

Remark 14. Instead of using $\mathrm{MI}_{1}$, a simplied criterion on $\oint^{2} \mathrm{H} \quad \oint_{k}^{2} \mathrm{H}$ or $\oint^{2} \mathrm{H} \quad \oint_{k \mathrm{k}}^{3} \mathrm{H}$ is sometimes incorrectly invoked. This is based on the deceptive guess that relevant conclusions may be derived from the consideration of the (articially uncoupled) 2 block of the Whitham matrix concerning the wave number and the amplitude (see for instance [Whi99, p.490]).

Remark 15. We recall that it was proved in [BGNR14] that the failure of weak hyperbolicity of the modulation system does imply a slow side-band ${ }^{14}$ instability of the background periodic wave, hence the use of the term modulational instability here. It follows from our analysis that such an instability occurs near the harmonic limit when the dispersionless system fails to be weakly hyperbolic or when $\operatorname{sign}\left(w_{0}\right) \quad m$ I is negative - recall from Corollary 4 and Remark 11 that the sign of $w_{0}$ dictates the one of in the harmonic limit. For this reason, given its practical importance, we make the latter sign more explicit in A ppendix A.

\subsection{Small wavenumber regime}

As in the harmonic regime, the $\mathrm{N}$ eigenvalues arising from those of $\mathrm{B} \mathrm{r}{ }_{U}^{2} \mathrm{H}\left(\mathrm{U}_{s} ; 0\right)$ may be analyzed by standard spectral perturbation analysis. We only need to blow up the two eigenvalues near $c$ and we shall do it by inverting and scaling $W \quad \mathrm{Cl}_{\mathrm{N}+2}$.

To do so we rst observe that, since $S=A B A^{\top}$ and $D_{S}=P_{S}{ }^{\top} S P_{S}$, we have

$$
\text { W } \quad \mathrm{Cl}_{\mathrm{N}+2}=\left(\mathrm{P}_{\mathrm{s}}^{\top} \mathrm{A}\right)^{1} \mathrm{kP}_{\mathrm{s}}^{\top}\left(\mathrm{r}_{; ; ;}^{2}\right) \mathrm{P}_{\mathrm{s}} \mathrm{D}_{\mathrm{S}}{ }^{1}{ }^{1} \mathrm{P}_{\mathrm{S}}^{\top} \mathrm{A}
$$

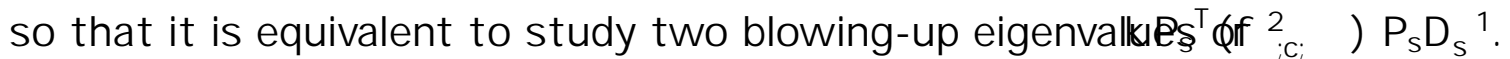

Now for concision's sake we introduce

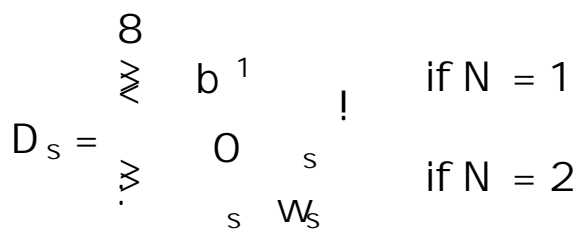

and

$$
x_{s}=\begin{array}{cccc}
8 & b_{s} b^{1} & \text { if } N=1 \\
3 & b_{s} s_{s} w_{s}+c_{s} s & \text { if } N=2
\end{array} \text { : }
$$

\footnotetext{
${ }^{14}$ That is, with small spectral parameter and small Floquet exponent.
} 
It follows from Theorem 2 and Corollary 3 that

$$
\begin{aligned}
& -P_{s}{ }^{\top}\left(r_{i ; ;}^{2}\right) \quad P_{s} D_{s}{ }^{1}
\end{aligned}
$$

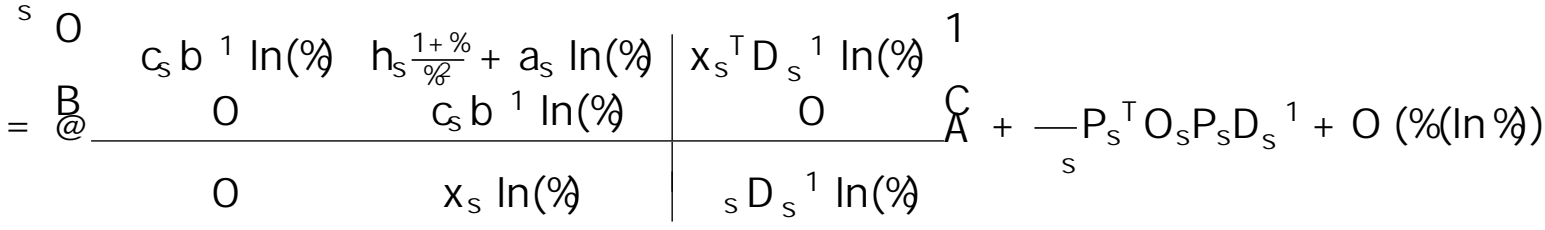

with

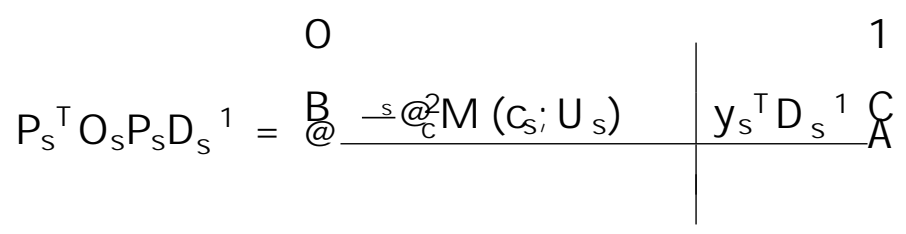

so that

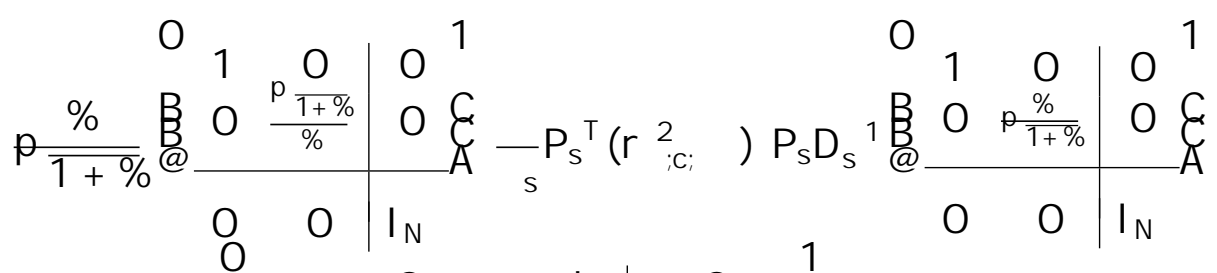

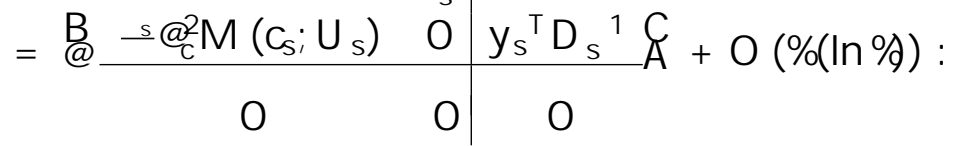

To the latter matrix we may apply elementary spectral perturbation analysis to study the two simple eigenvalues arising from $\quad h_{s}{ }_{s} @^{2} M=$ (where here ${ }^{-}$denotes any determination of the square root function).

Theorem 8. Under the assumptions of Theorem $4 \square$ consider $\left(c_{s} ; U_{s}\right) 2$ such that $Q_{s}^{2} M\left(c_{s} ; U_{s}\right) \in 0$. Then in the large period regime, the spectrum of the Whitham matrix $\left.W(k ; @)\left(c_{s} ; U_{s}\right) ; U_{s}\right)$ is given by

1. two eigenvalues expanding as ${ }^{15}$

$$
c_{s} \quad \frac{\%^{p}-}{\overline{\left.h_{s} \quad{ }_{s} @_{2}^{2} M\left(c_{s} ; U_{s}\right) ; U_{s}\right)}}+0(\% \ln (\%)
$$

(where here ${ }^{\mathrm{p}-}$ denotes some determination of the square root function), with cor-

\footnotetext{
${ }^{15}$ We recall that $k=(s \ln (\%)$ in the solitary wave limit.
} 
responding eigenvectors ${ }^{16}$

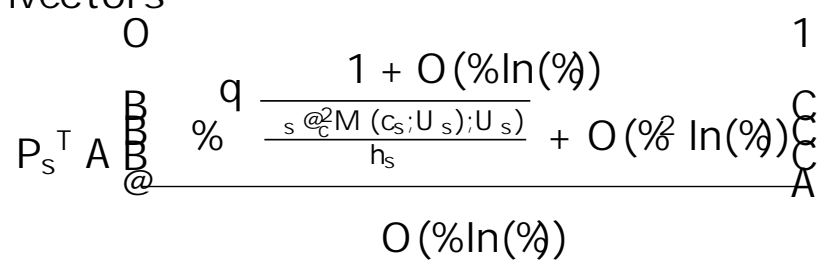

2. and $\mathrm{N}$ eigenvalues expanding as

$$
z_{j}+O(k) ; \quad \text { j } 2 \text { f } 1 ; N g
$$

with associate eigenvectors

$$
\begin{aligned}
& { }^{0} r_{U} M^{\top}\left(r{ }_{U}^{2} H(U 0 ; 0)+C_{s} B{ }^{1}\right){ }^{1} B{ }^{1} r_{j}+O(k)^{1} \\
& \text { @ } \quad 0(\mathrm{k}) \quad \text { ह } \quad j 2 \mathrm{f} 1 ; \mathrm{Ng} \\
& r_{j}+O(k)
\end{aligned}
$$

where $z_{j}, j 2 f 1 ; N g$, are the distinct and real eigenvalues of $\mathrm{Br} \underset{U}{2} \mathrm{H}\left(\mathrm{U}_{s} ; 0\right)$, with corresponding eigenvectors, $r_{j}$, j $2 f 1 ; \mathrm{Ng}$.

Moreover all the bounds are locally uniform with respect to $\left(\mathrm{C}_{s} ; \mathrm{U}_{\mathrm{s}}\right)$.

Remark 16. Though diagonilizability of the limiting modulation systems has little direct impact on the hyperbolicity of modulation systems near the limit, in the reverse direction the expansions derived in Theorems 7 and 8 Shed some light on the asymmetry between the harmonic and the soliton limits in terms of diagonalizability of the asymptotic systems. Indeed, in the latter limit, the convergence of the eigenvalues towards the double root occurs exponentially faster | as $\% \mathrm{n} \%$ | than the convergence of eigenvectors, which converge as $1=\ln \%$ and this may be proved to imply per se persistence of diagonalizability at the limit. In contrast, in the former limit the perturbations of eigenvectors and eigenvalues are of the same order | namely | leaving room for a limiting Jordan block.

\section{A ppendix}

A Explicit formula for the modulational-instability index

The goal of this section is to make explicit both $\theta_{0}$ and $M_{1}$ that are involved in the hyperbolicity of the Whitham system near or at the harmonic limit.

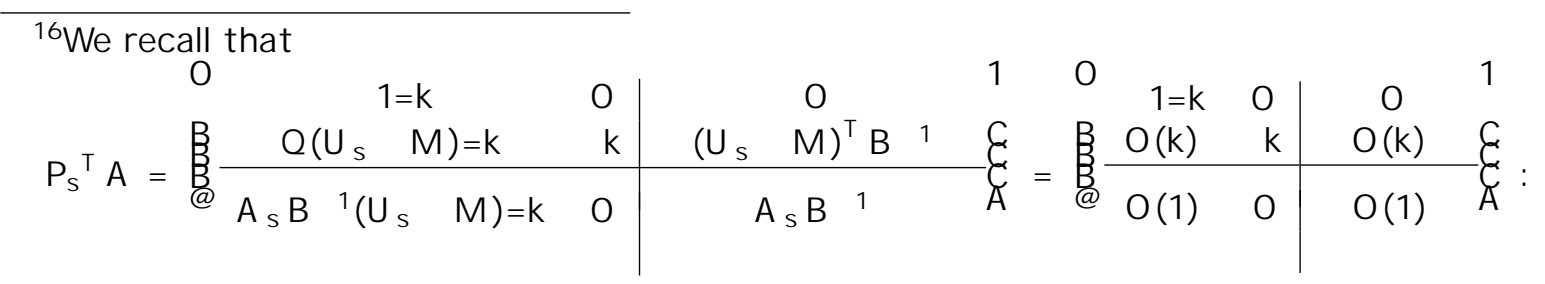


This requires the extraction from [BGMRarl of an explicit value for the coecient in Theorem 2 (denoted 0 in [BGMRarl]). First we recall from [BGMRar] that

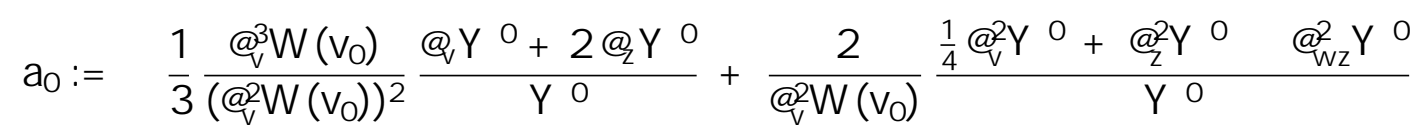

where

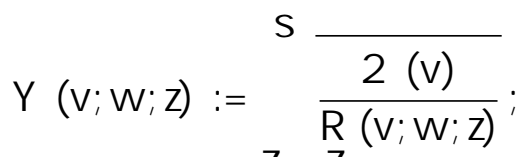

$$
\begin{aligned}
& R(v ; w ; z):=\underbrace{Z_{1} Z_{1}}_{0} t^{2} W\left(w+t\left(\begin{array}{ll}
z & w
\end{array}\right)+t s\left(\begin{array}{ll}
v & z
\end{array}\right)\right) d s d t:
\end{aligned}
$$

Here we omit to specify the dependence of $W, R$ and $Y$ on parameters $\left(c_{0} ; 0\right)$ since they are held xed along the computation, and the exponent ${ }^{0}$ denotes that functions of $(v ; w ; z)$ are evaluated at $\left(v_{0} ; v_{0} ; v_{0}\right)$.

First we recall from [BGMRar, $A$ ppendix $B$ ] that $R$ is a symmetric function and we observe that

$$
\begin{aligned}
& \mathrm{R}^{0}=\frac{1}{2} @^{2} \mathrm{~W}\left(\mathrm{v}_{0}\right) ; \\
& Q_{1} R^{0}=\frac{1}{6} @^{3} W\left(v_{0}\right) ; \\
& Q_{1}^{2} R^{0}=\frac{1}{12} Q^{4} W\left(v_{0}\right) \text {; } \\
& \varrho_{w z}^{2} R^{0}=\frac{1}{24} \varrho_{w}^{4} W\left(v_{0}\right):
\end{aligned}
$$

M oreover direct computations yield

$$
\begin{aligned}
& \frac{Q^{0}}{Y^{0}}=\frac{1}{2} \frac{9\left(v_{0}\right)}{\left(v_{0}\right)} \quad \frac{1}{2} \frac{R^{0}}{R^{0}} ; \\
& \frac{Q^{0}}{Y^{0}}=\frac{1}{2} \frac{R^{0}}{R^{0}} \\
& \frac{@_{0} Y^{0}+2 @ Y^{0}}{Y^{0}}=\frac{1}{2} \frac{9\left(v_{0}\right)}{\left(v_{0}\right)} \quad \frac{3}{2} \frac{Q_{1}^{0} R^{0}}{R^{0}}=\frac{1}{2} \frac{9\left(v_{0}\right)}{\left(v_{0}\right)} \quad \frac{1}{2} \quad \frac{\Theta^{3} W\left(v_{0}\right)}{\Theta_{0}^{2} W\left(v_{0}\right)} ;
\end{aligned}
$$

and

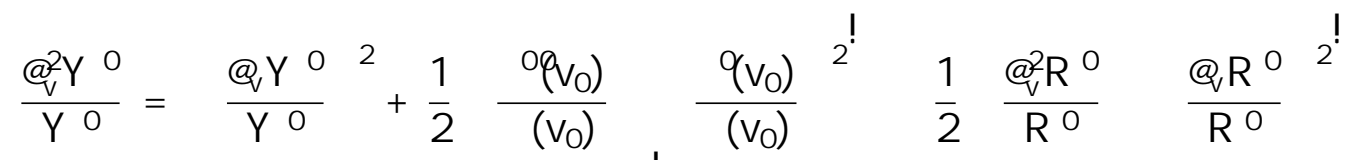

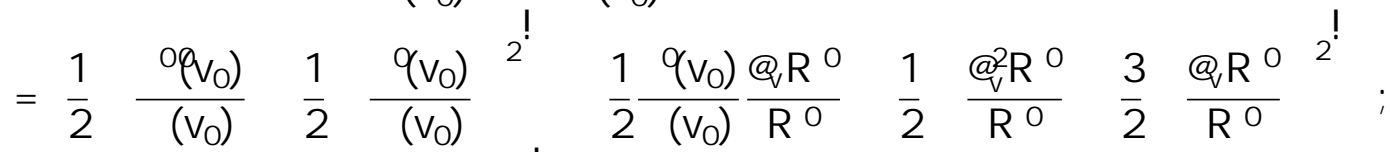

$$
\begin{aligned}
& \frac{@_{2}^{2} Y^{0}}{Y^{0}}=\frac{1}{2} \frac{@_{1}^{2} R^{0}}{R^{0}} \quad \frac{3}{2} \frac{Q_{1} R^{0}}{R^{0}} \quad \text {; }
\end{aligned}
$$

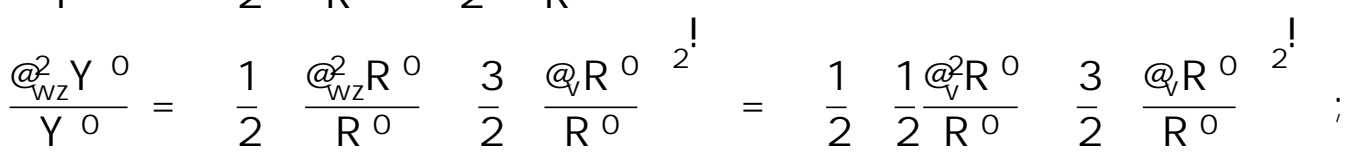


so that

$$
\begin{aligned}
& \frac{\frac{1}{4} @_{Y}^{2} Y^{0}+@_{Z}^{2} Y^{0} \bigotimes_{w Z}^{2} Y^{0}}{Y^{0}}=
\end{aligned}
$$

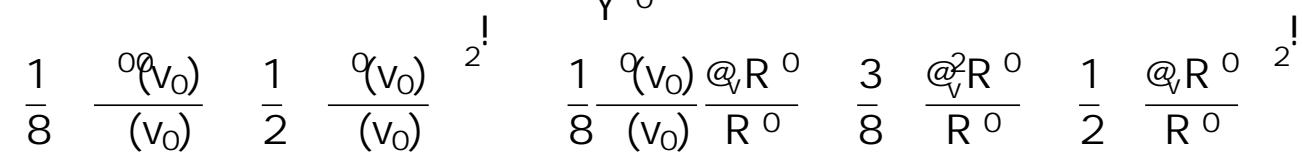

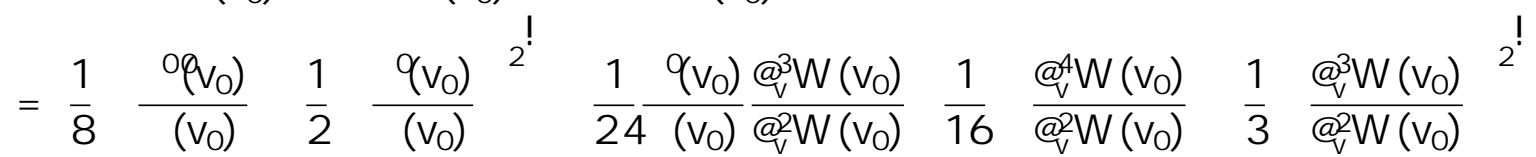

thus

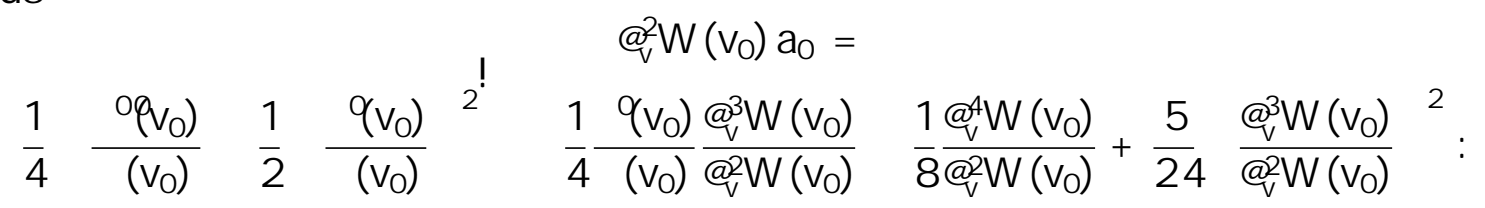

To go further with computations we nd it convenient to separate the scalar and system case.

\section{A.1 The scalar case}

In the scalar case, note that the computations in the proof of Corollary 5 provide

$$
\begin{aligned}
@_{0}^{2} W\left(v_{0}\right) & \left.=f 0 q v_{0}\right) \quad \frac{c_{0}\left(k_{0} ; v_{0}\right)}{b}=(2)^{2} k_{0}^{2}\left(v_{0}\right) \\
b @ H\left(v_{0} ; 0\right)+c_{0}\left(k_{0} ; v_{0}\right) & =b @ W\left(v_{0}\right) \\
b @ H\left(v_{0} ; 0\right)+v_{g}\left(k_{0} ; v_{0}\right) & =3 b @ W\left(v_{0}\right)
\end{aligned}
$$

and observe that when ' $3, @ W\left(v_{0}\right)=f^{(')}\left(v_{0}\right)$. From this one readily derives

$$
\begin{aligned}
k_{0}\left(2 @ c_{0}\left(k_{0} ; v_{0}\right) \quad k_{0} @ c_{0}\left(k_{0} ; v_{0}\right)\right. & \left.=2 b @ W\left(k_{0} ; v_{0}\right)\right)=6 b @ W\left(v_{0}\right) ; \\
@_{10} c_{0}\left(k_{0} ; v_{0}\right) & =b @ W\left(v_{0}\right) \quad b \frac{9\left(v_{0}\right)}{\left(v_{0}\right)} @^{2} W\left(v_{0}\right) ;
\end{aligned}
$$

so that

$$
\begin{aligned}
& \frac{a_{0}}{b^{2} k_{0}^{2} @_{0}^{2} W\left(v_{0}\right)}=4 @_{1}^{2} W\left(v_{0}\right) a_{0}+\frac{2}{3} \frac{@^{3} W\left(v_{0}\right)}{@^{2} W\left(v_{0}\right)} \quad \frac{9\left(v_{0}\right)}{\left(v_{0}\right)}
\end{aligned}
$$

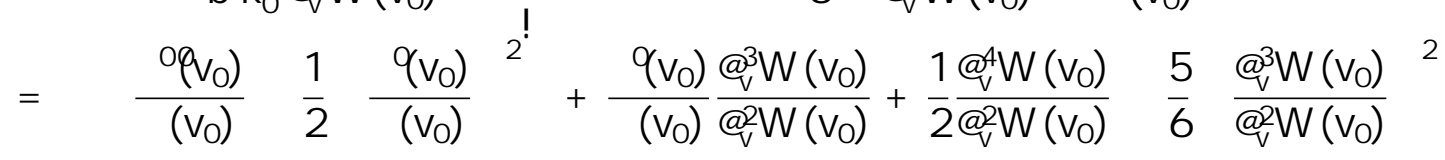

$$
\begin{aligned}
& =\frac{\left.0 v_{0}\right)}{\left(v_{0}\right)}+\frac{5}{6} \frac{9\left(v_{0}\right)}{\left(v_{0}\right)}+\frac{1}{3} \frac{\rho\left(v_{0}\right)}{\left(v_{0}\right)} \frac{f^{\left.00 \rho v_{0}\right)}}{(2)^{2} k_{0}^{2}\left(v_{0}\right)} \frac{1}{6} \frac{f^{\left.00 \rho v_{0}\right)}}{(2)^{2} k_{0}^{2}\left(v_{0}\right)}+\frac{1}{2} \frac{f^{\left.009 q_{0}\right)}}{(2)^{2} k_{0}^{2}\left(v_{0}\right)} \text { : }
\end{aligned}
$$

From the foregoing computations we also derive that

$$
M I=a_{0} 2 @ c_{0}+k_{0} @^{2} c_{0}=6 b^{3} k_{0}\left(@^{2} W\left(v_{0}\right)\right)^{2}
$$




$$
\text { " } \frac{\left.0 \varphi v_{0}\right)}{\left(v_{0}\right)} \quad \frac{5}{6} \frac{9\left(v_{0}\right)}{\left(v_{0}\right)}{ }^{2} \quad \frac{1}{3} \frac{\left(v_{0}\right)}{\left(v_{0}\right)} \frac{\left.f 00 \rho v_{0}\right)}{(2)^{2} k_{0}^{2}\left(v_{0}\right)}+\frac{1}{6} \frac{\left.f 00 \rho v_{0}\right)}{(2)^{2} k_{0}^{2}\left(v_{0}\right)} \quad \frac{1}{2} \frac{\left.f 009 q_{0}\right)}{(2)^{2} k_{0}^{2}\left(v_{0}\right)} \text { : }
$$

Recall from Proposition 27that in the scalar case the sign of is given by the sign of $b$ so that we are interested in the sign of $b \quad \mathrm{~m}$. We stress moreover that this sign may be determined by considering a second-order polynomial in the unknown $\mathrm{k}_{0}^{2}$, that varies in $(0 ; 1)$, with coecients depending on $v_{0}$.

We leave this general discussion to the reader and focus now on the most classical case when is constant. To begin, note that for the 'genuine' Korteweg-de Vries equation, $f$ is cubic and is constant so that both $a_{0}$ and $b \quad m$ I are positive. Likewise, when is constant, we have

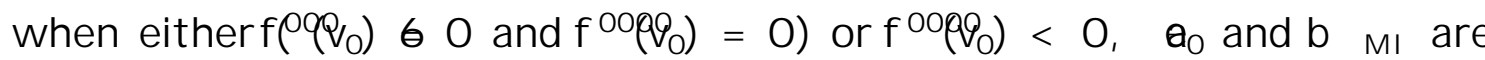
positive;

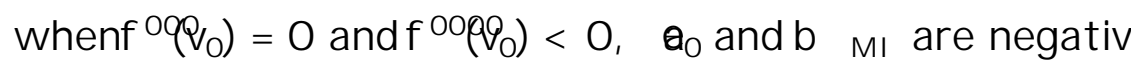

when $\left.f 00 \rho v_{0}\right) \in 0$ and $f{ }^{009}\left(v_{0}\right)<0$, the common sign of $a_{0}$ and $b \quad m_{1}$ depends on the harmonic wavenumber $k_{0}$, modulational instability occurring for wavenumbers $\mathrm{k}_{0}$ larger than the critical wavenumber

$$
k_{c}\left(v_{0}\right):=p_{\overline{3}}^{1} \frac{p \text { jf }{ }^{\left.00 \rho v_{0}\right) j}}{\left(v_{0}\right) \text { jf } 00 q\left(v_{0}\right) j}:
$$

It is worth pointing out that the general case when is arbitrary is richer and that there are situations when two critical wavenumbers appear in the analysis.

\section{A.2 The system case}

As a preliminary to computations in the system case, we recall that

b (v) $g(v ; c ;)=c v \quad b$;

$$
W(v ; c ; \quad)=f(v) \quad \frac{1}{2}(v)(g(v ; c ; 2))^{2} \quad \frac{c}{b} v g(v ; c ; 2) \quad(v ; g(v ; c ; 2)) \text {; }
$$

so that

b (v) @g(v;c; ) = c b $9(v) g(v ; c ;)$;

$b(v) @ g(v ; c ;)=b 0 q v) g(v ; c ;) 2 b 9(v) @ g(v ; c ;)$;

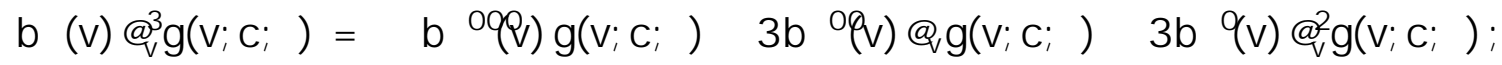


and

$$
\begin{aligned}
& @ W(v ; c ;)=f 9(v) \quad \frac{1}{2} 9(v)(g(v ; c ; 2))^{2} \quad \frac{c}{b} g(v ; c ; 2) \quad 1 ; \\
& @^{2} W(v ; c ; \quad)=\oint^{2} H((v ; g(v ; c ; 2)) ; 0)+(v)(@ g(v ; c ; 2))^{2} \text {; } \\
& \left.@^{3} W(v ; c ; \quad)=@^{3} H((v ; g(v ; c ; 2)) ; 0) \quad 309 v\right) g(v ; c ; 2) @ g(v ; c ; 2) \\
& 39(v)(@ g(v ; c ; 2))^{2} \text {; } \\
& \left.\oint^{4} W(v ; c ; \quad)=\oint_{0}^{4} H((v ; g(v ; c ; 2)) ; 0) \quad 4 \quad 00 \rho v\right) g(v ; c ; 2) @ g(v ; c ; 2) \\
& \left.609 v)(@ g(v ; c ; 2))^{2} \quad 3 \quad 09 v\right) g(v ; c ; 2) @ 2 g(v ; c ; 2) \\
& 69(v) @ g(v ; c ; 2) @ g(v ; c ; 2) \text { : }
\end{aligned}
$$

In particular, it follows recursively that for ' $2, @_{1}^{\prime} g(v ; C ; 2)$ and @ $W(v ; C ; \quad)$ may be written as functions of $v, g(v ; c ; 2)$ and $@ g(v ; c ; 1)$, independently of and $c$. We also observe, essentially as in the proof of Corollary 5 that

$$
\begin{aligned}
& \operatorname{tr}\left(B r{ }_{U}^{2} H((v ; g(v ; c ;)) ; 0)+c l_{N}\right)=2 b(v) @ g(v ; c ; 2) \text {; }
\end{aligned}
$$

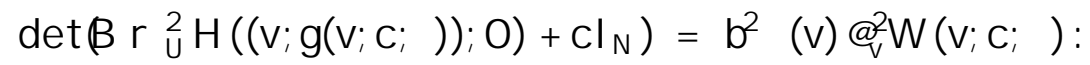

Now we point out that $c_{0}\left(k_{0} ; U_{0}\right)$ is dened by

$$
\operatorname{det}\left(B r \underset{U}{2} H\left(U_{0} ; 0\right)+c_{0}\left(k_{0} ; U_{0}\right) I_{N}\right)=b^{2}\left(v_{0}\right)(2)^{2} k_{0}^{2}\left(v_{0}\right)
$$

more explicitly written as

$$
\left(c_{0}\left(k_{0} ; U_{0}\right)\right)^{2}+c_{0}\left(k_{0} ; U_{0}\right) \operatorname{tr}\left(B r \underset{U}{2} H\left(U_{0} ; 0\right)\right)+\operatorname{det}(B r \underset{U}{2} H(U 0 ; 0))=b^{2}\left(v_{0}\right)(2)^{2} k_{0}^{2}\left(v_{0}\right)
$$

and that this denition makes sense if and only if

$$
k_{0}^{2} \frac{\operatorname{det}\left(B r{ }_{U}^{2} H\left(U_{0} ; 0\right)\right) \frac{1}{4}\left(\operatorname{tr}\left(B r \underset{U}{2} H\left(U_{0} ; 0\right)\right)\right)^{2}}{b^{2}\left(v_{0}\right)(2)^{2}\left(v_{0}\right)}=\frac{\left.\left.f 0 q v_{0}\right)+\frac{1}{2} 0 q_{v_{0}}\right) u_{0}^{2}}{(2)^{2}\left(v_{0}\right)} \text { : }
$$

Yet the latter prescribes a minimal value for $k_{0}$ only if $\left.\left.f 0 q v_{0}\right)+\frac{1}{2} \quad 0 q v_{0}\right) u_{0}^{2}<0$, that is, only if the corresponding dispersionless system already fails to be hyperbolic. Moreover when the inequality on $\mathrm{k}_{0}^{2}$ is strictly satised there are actually two possible values for $c_{0}\left(k_{0} ; U_{0}\right)$. This denes two branches for $c_{0}$ and henceforth we follow one such branch.

By dierentiating the relation dening $\quad c_{0}$ with respect to $k$ we derive

$$
\begin{aligned}
& @ g\left(v_{0}\right) k_{0} @ c_{0}\left(k_{0} ; U_{0}\right)=b @ W\left(v_{0}\right) ; \\
& @ g\left(v_{0}\right) k_{0}^{2} @_{k}^{2} c_{0}\left(k_{0} ; U_{0}\right)=b @ W\left(v_{0}\right)+\frac{k_{0}^{2}\left(@ c_{0}\left(k_{0} ; U_{0}\right)\right)^{2}}{b\left(v_{0}\right)} ; \\
& \left(2 @ c_{0}\left(k_{0} ; U_{0}\right) \quad k_{0} @^{2} c_{0}\left(k_{0} ; U_{0}\right)\right)=\frac{b @ W\left(@^{2} W+3(@ g)^{2}\right)}{k_{0}(@ g)^{3}} ;
\end{aligned}
$$

(where again here and from now on we omit to mark dependencies on $\mathrm{c}$ and on $\mathrm{g}$ and W ). 
At this stage, we could dierentiate with respect to $U$ to compute $r \cup \mathrm{C}_{0}\left(\mathrm{k}_{0} ; \mathrm{U}_{0}\right)$ and conclude as in the scalar case. Yet, instead we shall directly use the relatively explicit formula derived in Subsection 4.3. The only missing piece to carry out this task is to extract from [BGMRar] $a$ formula for the coecient $\quad b_{0}$ from Theorem 2 (denoted 0 in [BGMRar]). With notation introduced above,

$$
\begin{aligned}
& b_{0}:=\frac{1}{3} \frac{\varrho^{3} W\left(v_{0}\right)}{\left(@_{0}^{2} W\left(v_{0}\right)\right)^{2}}+\frac{1}{@_{0}^{2} W\left(v_{0}\right)} \frac{@^{0} Y^{0}}{Y^{0}}
\end{aligned}
$$

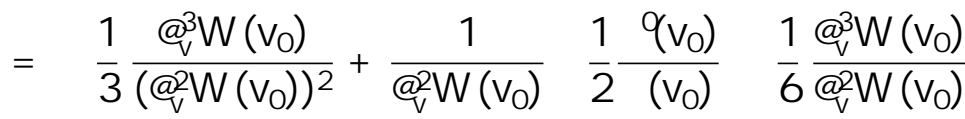

$$
\begin{aligned}
& =\frac{1}{2} \frac{1}{\Theta_{2}^{2} W\left(v_{0}\right)} \frac{9\left(v_{0}\right)}{\left(v_{0}\right)} \frac{\Theta_{0}^{3} W\left(v_{0}\right)}{@_{0}^{2} W\left(v_{0}\right)}:
\end{aligned}
$$

Now with notation from Subsection 43

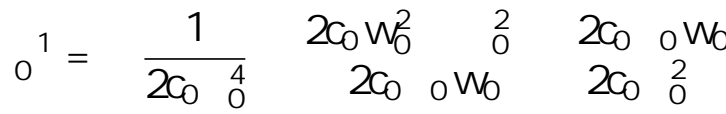

$$
\begin{aligned}
& \frac{\mathrm{k}_{0}^{2}}{\mathrm{c}_{0}^{2} \mathrm{w}_{0}^{2}} \mathrm{x}_{0}^{\top} \quad{ }_{0}^{1} \mathrm{x}_{0}=\frac{\mathrm{k}_{0}^{2}}{2 \mathrm{c}_{0}^{3} \mathrm{w}_{0}^{2}} \quad \mathrm{~b}_{0}^{2}+2 \mathrm{c}_{0}^{3} \frac{\mathrm{o}_{0}^{2}}{0_{0}^{2}} \\
& =\frac{\frac{1}{4} b^{2} k_{0}^{2}}{\bigotimes_{0}^{2} W(@ g)^{2}} \quad-\quad @^{2} W \quad \bigotimes^{3} W^{2}+\left(\bigotimes_{0}^{2} W\right) \quad\left(@^{2} g\right)^{2}
\end{aligned}
$$

thus at the harmonic limit

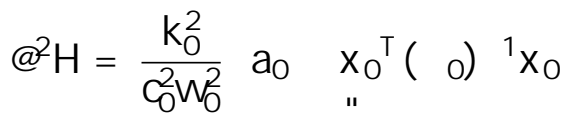

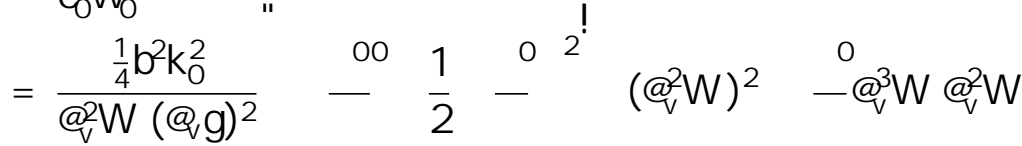

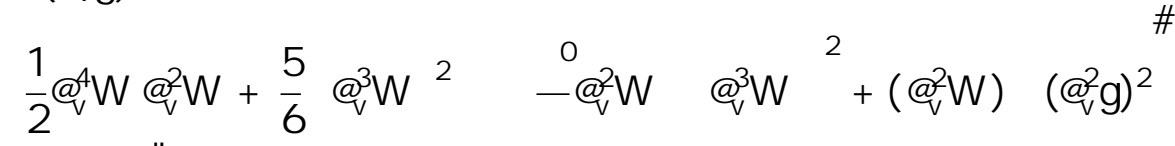

$$
\begin{aligned}
& =\frac{\frac{1}{4} b^{2} k_{0}^{2}}{@^{2} W(@ g)^{2}} \quad \frac{1}{2} @^{4} W @^{2} W \quad \frac{1}{6} @^{3} W^{2}+-@^{3} W @^{2} W \\
& +\quad-\frac{3}{2}-^{0} 2^{!}\left(@^{2} W\right)^{2}+\left(@^{2} W\right)\left(@^{2} g\right)^{2}:
\end{aligned}
$$

To proceed we now consider at the harmonic limit

$$
\begin{aligned}
& \left(@ r_{M} H\right)^{\top}\left(r_{M}^{2} H \quad\left(@^{2} H\right) B^{1}\right)^{1} @ r_{M} H \\
& =\frac{\mathrm{k}_{0}^{2}}{\mathrm{C}_{0}^{2} \mathrm{w}_{0}^{2}} \mathrm{X}_{0}^{\top}(0){ }^{1} \mathrm{~A}_{0} \mathrm{~B}^{1} \mathrm{~B}^{1} \mathrm{~A}_{0}^{\top}(0){ }^{1} \mathrm{~A}_{0} \mathrm{~B}^{1}+\frac{1}{\mathrm{C}_{0} \mathrm{~W}_{0}} \mathrm{~B}^{1}{ }^{1} \mathrm{~B}^{1} \mathrm{~A}_{0}{ }^{\top}(\quad 0){ }^{1} \mathrm{X}_{0}
\end{aligned}
$$


and observe that on one hand

$$
\begin{aligned}
& A_{0} B^{1}=\begin{array}{rr}
0 & 0 \\
\frac{w_{0}}{2} & \frac{1}{b}
\end{array} \\
& B^{1} A_{0}{ }^{\top}\left(0^{2}\right)^{1} A_{0} B^{1}=\frac{1}{2 c_{0}{ }_{0}^{2}} \quad{ }^{\frac{c_{0} w_{0}^{2}}{2}} \frac{c_{0} w_{0}}{b}{ }^{2} \frac{c_{0} w_{0}}{\frac{2 c_{0}}{b^{2}}}
\end{aligned}
$$

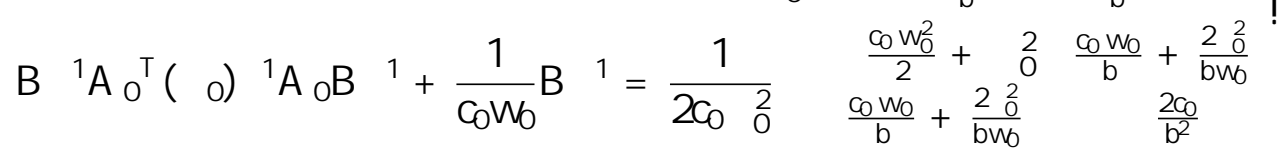

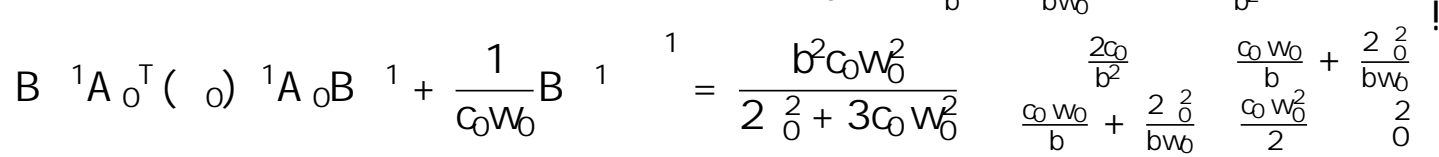

and that on the other hand

$$
\begin{aligned}
& \text { ( o) }{ }^{1} \mathrm{x}_{0}=\frac{1}{2 c_{0}{ }_{0}^{3}} \quad \begin{array}{llllllll}
b_{0} & { }_{0}^{2} & 2 c_{0}^{2} & 0 w_{0} \\
2 c_{0}^{2} & 0 & 0
\end{array} \\
& \text { B }{ }^{1} A_{0}{ }^{\top}\left(0^{1}\right)^{1} \mathrm{X}_{0}=\frac{1}{2 c_{0}{ }_{0}^{2}} \quad b_{0}{ }_{0}^{2}+\frac{2 c_{0}^{2} 0 W_{0}}{\frac{2 c_{0}^{2} 0}{b}} \text { : }
\end{aligned}
$$

Thus

$$
\begin{aligned}
& \left(@ r_{M} H\right)^{\top}\left(r_{M}^{2} H \quad\left(@_{N_{11}^{2}}^{2} H\right) B^{1}\right)^{1} @ r_{M} H \\
& =\frac{b^{2} k_{0}^{2}}{4 c_{0}^{3}{ }_{0}^{4}\left(2{ }_{0}^{2}+3 c_{0} w_{0}^{2}\right)} \frac{2 c_{0}}{b^{2}}\left(b_{0}{ }_{0}^{2}+c_{0}^{2}{ }_{0} w_{0}\right)^{2} \quad \frac{4 c_{0}^{2} 0}{b}\left(b_{0}{ }_{0}^{2}+c_{0}^{2}{ }_{0} w_{0}\right) \quad \frac{c_{0} w_{0}}{b}+\frac{22_{0}^{2}}{b w_{0}} \\
& +\frac{4 c_{0}^{4} 0_{0}^{2}}{b_{11}^{2}} \frac{c_{0} w_{0}^{2}}{2} \quad 2
\end{aligned}
$$

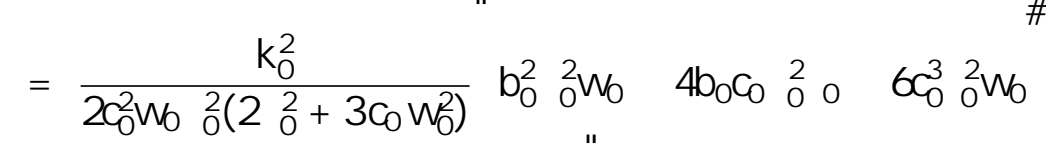

$$
\begin{aligned}
& =\frac{\frac{1}{4} b^{2} k_{0}^{2}}{@ g\left(@_{0}^{2} W\right)\left(@^{2} W+3 \quad(@ g)^{2}\right)} @ g-@^{2} W \quad @^{3} W^{2}
\end{aligned}
$$

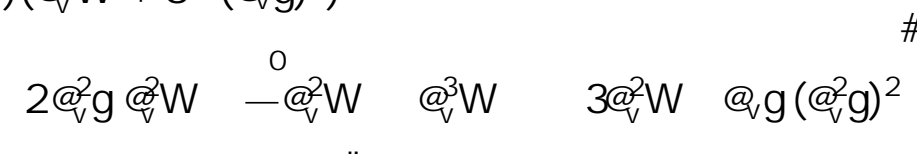

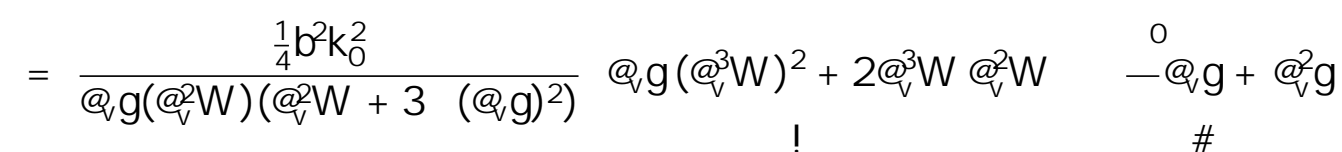

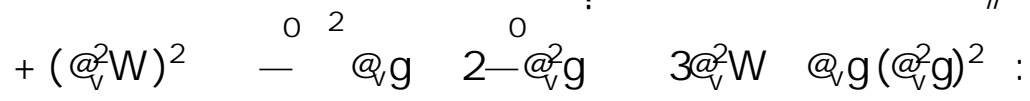


Finally

$$
\begin{aligned}
& a_{0}=@^{2} H \quad\left(@ r_{M} H\right)^{\top}\left(r_{M}^{2} H \quad\left(@_{M}^{2} \underset{n}{H}\right) B^{1}\right)^{1} @ r_{M} H \\
& =\frac{\frac{1}{4} b^{2} k_{0}^{2}}{(@ g)^{2}\left(@^{2} W\right)\left(@^{2} W+3(@ g)^{2}\right)} \quad(@ g)^{2}\left(@^{3} W\right)^{2} \\
& +2 @ @^{3} W @^{2} W \quad(@ g) \quad-@ g+@^{2} g
\end{aligned}
$$

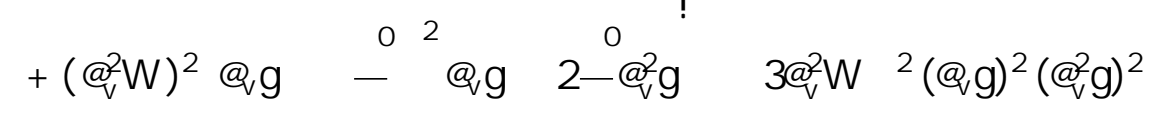

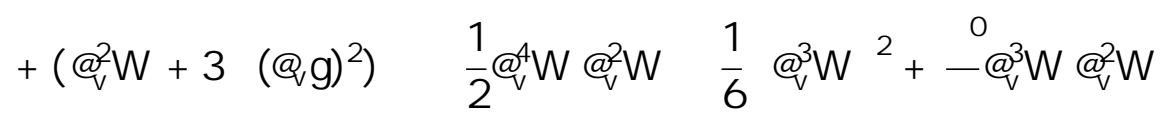

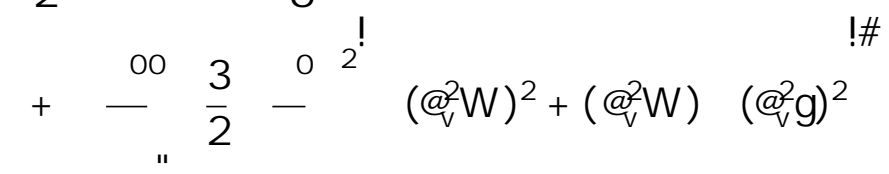

$$
\begin{aligned}
& =\frac{\frac{1}{4} b^{2} k_{0}^{2}}{(@ g)^{2}\left(@^{2} W\right)\left(@^{2} W+3(@ g)^{2}\right)} \quad \frac{1}{2} @^{4} W @^{2} W\left(@^{2} W+3 \quad(g)^{2}\right) \\
& \frac{1}{6} @^{3} W^{2}\left(@^{2} W \quad 3(@ g)^{2}\right)+@^{3} W @^{2} W \quad-\left(@^{2} W+(@ g)^{2}\right)+2(@ g) @^{2} g \\
& +\quad-\frac{3}{2} 0^{2}\left(@^{2} W\right)^{3}
\end{aligned}
$$

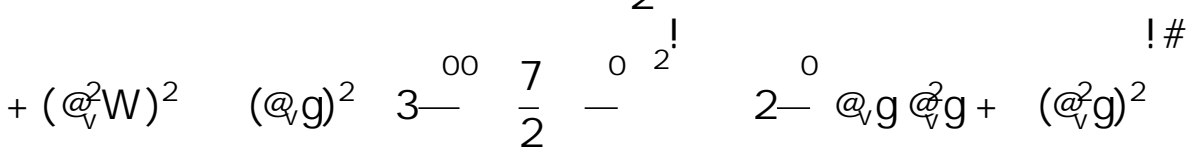

and

$$
\begin{aligned}
& \frac{4(@ g)^{5}}{b^{3} k_{0}} \frac{@^{2} W+3(@ g)^{2}}{@^{2} W+3(@ g)^{2}} \quad \text { M I } \\
& =\frac{1}{2} @^{4} W @^{2} W\left(@^{2} W+3(@ g)^{2}\right) \\
& \frac{1}{6} @^{3} W^{2}\left(@^{2} W \quad 3(@ g)^{2}\right)+@^{3} W \quad @^{2} W \quad-\left(@^{2} W+(@ g)^{2}\right)+2 \quad(@ g) @^{2} g \\
& +\quad-\frac{3}{2} 0^{2}\left(\varrho^{2} W\right)^{3}
\end{aligned}
$$

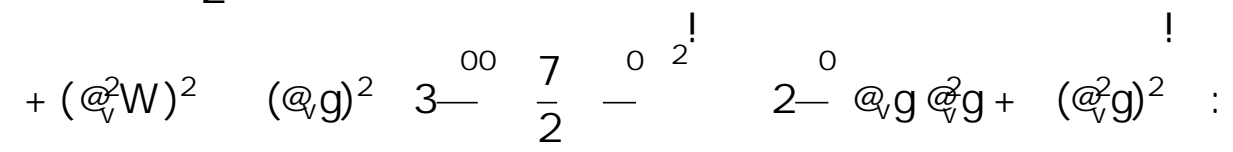

Recall that is of the sign of $w_{0}=2 @ g=b s o$ that this is the sign of $@_{g} g \quad m_{1}=b$, hence of the quantity written above, that matters here. We observe that in order to write this 
criterion directly in terms of $\left(\mathrm{k}_{0} ; \mathrm{U}_{0}\right)$, one may use that

$$
\begin{aligned}
& @_{0}^{2} W\left(v_{0}\right)=\left(\begin{array}{lll}
2 & )^{2} k_{0}^{2} & \left(v_{0}\right) ;
\end{array}\right. \\
& Q_{1} g\left(v_{0}\right)=p \frac{1}{\left(v_{0}\right)} \stackrel{q}{@_{0}^{2} H\left(U_{0} ; 0\right)+(2 \quad)^{2} k_{0}^{2}\left(v_{0}\right)}
\end{aligned}
$$

with the sign choice corresponding to the choice of a branch for $\mathrm{c}_{0}\left(\mathrm{k}_{0} ; \mathrm{U}_{0}\right)$ and that all other quantities have already been expressed in terms of $U_{0}$ and $@_{1} g\left(v_{0}\right)$. Note however that since @ $g\left(v_{0}\right)$, thus $c_{0}$, is not a polynomial function of $k_{0}$ the range of possibilities is signicantly harder to analyze in terms of $\left(\mathrm{k}_{0} ; \mathrm{U}_{0}\right)$ than in the scalar case. It may be preferable instead to express the criterion in terms of $\left(U_{0} ; @_{;} g\left(v_{0}\right)\right)$.

Alternatively, since the general computations are somewhat tedious, from now on we shall rather make the extra assumption, satised by the most standard cases that is ane. This ensures that the expression to study is indeed a polynomial in $k_{0}^{2}$ with coecients depending on $U_{0}$. In this direction, note that in this case

$$
\begin{aligned}
& \left(@ g\left(v_{0}\right)\right)^{2}=f^{09}\left(v_{0}\right)+Q^{2} W\left(v_{0}\right) \text {; } \\
& @^{2} g\left(v_{0}\right)=2 \frac{9\left(v_{0}\right)}{\left(v_{0}\right)} @ g\left(v_{0}\right) \text {; } \\
& \left.\bigotimes^{3} W\left(v_{0}\right)=f^{00 \rho} v_{0}\right) \quad 3 \frac{9\left(v_{0}\right)}{\left(v_{0}\right)}\left(v_{0}\right)\left(@ g\left(v_{0}\right)\right)^{2} ; \\
& @^{4} W\left(v_{0}\right)=f^{\left.009 q_{0}\right)+12} \frac{q\left(v_{0}\right)^{2}}{\left(v_{0}\right)} \quad\left(v_{0}\right)\left(@ g\left(v_{0}\right)\right)^{2}:
\end{aligned}
$$

Thus under the same assumption the range of admissible parameters is described by 


$$
\begin{aligned}
& @^{2} \mathrm{~W} \quad \mathrm{f}^{00} \text { and we have } \\
& \begin{array}{l}
\frac{4(@ g)^{5}}{b^{3} k_{0}} \frac{4 @^{2} W+3 f^{00}}{2 @^{2} W+3 f^{00}} \\
=\frac{1}{2} f^{0000} 6-0^{2}\left(@^{2} W+f 0 q \quad @^{2} W\left(4 @^{2} W+3 f^{09}\right.\right.
\end{array} \\
& +\frac{1}{6} f^{000}+3-\left(@^{2} w+f 0 q^{2}\left(2 @^{2} w+3 f 0 q\right.\right. \\
& f^{000}+3-\left(@^{2} W+f 09 \quad @^{2} W \quad-\left(2 @^{2} w+f 09 \quad 4-\left(@^{2} w+f 09\right.\right.\right. \\
& +\quad-\frac{3}{2} 0^{2}\left(@^{2} W\right)^{3}
\end{aligned}
$$

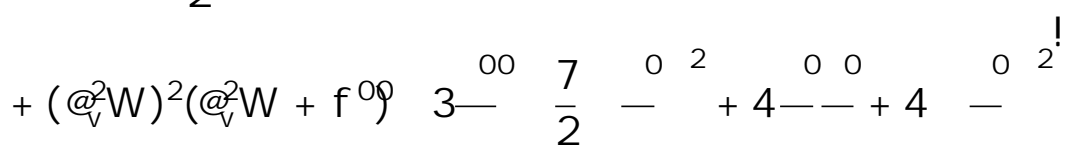

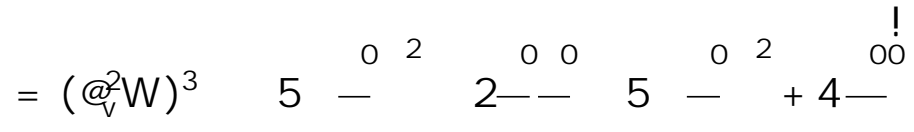

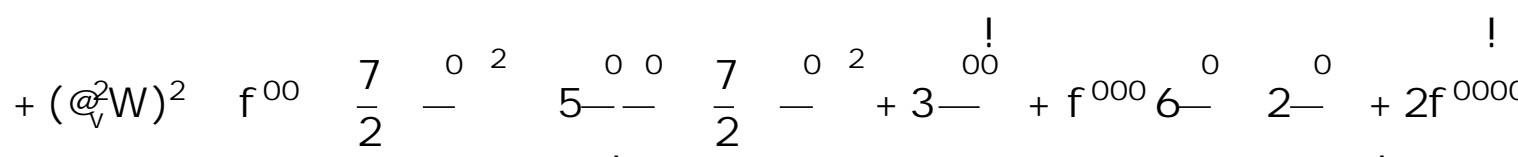

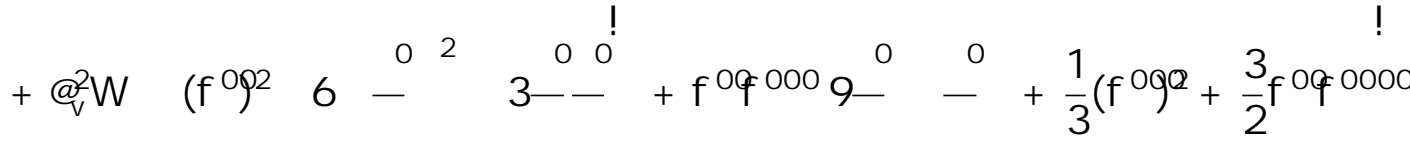

$$
\begin{aligned}
& +\frac{1}{2} f^{00} f^{000}+3-f^{00}{ }^{2}
\end{aligned}
$$

Recall that $\oint_{0}^{2} \mathrm{~W}\left(\mathrm{v}_{0}\right)=\left(\mathrm{v}_{0}\right)(2)^{2} \mathrm{k}_{0}^{2}$ so that the latter expression is indeed a third-order polynomial expression in $k_{0}^{2}$ with coecients depending on $v_{0}, k_{0}^{2}$ being allowed to vary in $\left.\left.\left(\max \left(f 0 ; \quad f 09 v_{0}\right)=\left(v_{0}\right)(2)^{2}\right) g\right) ; 1\right)$ and that this is negativity of the expression that yields modulational instability.

$N$ ote that if one specializes to the cases arising from the hydrodynamic formulation of a nonlinear Schr odinger equation (see [BG13] for instance)

$$
\mathrm{i} @=@_{x}^{2}+\mathrm{f}^{0}\left(\mathrm{j} \mathrm{j}^{2}\right) \text {; }
$$

then $=I d$ and is given by $(v)=1=(4 v)$ so that the foregoing expression is reduced to $v_{0}{ }^{2}$ times the second-order polynomial

$$
\begin{aligned}
& \left(@^{2} W\right)^{2} \quad 4 v_{0}{ }^{2} f 00+8 v_{0}{ }^{1} f 000+2 f 0000 \\
& +@_{j}^{2} W \quad 9 v_{0}{ }^{2}\left(f^{0} q^{2}+10 v_{0}{ }^{1} f 00 f 000+\frac{1}{3}\left(f^{00}\right)^{2}+\frac{3}{2} f^{00} f 0000\right. \\
& +\frac{1}{2} f^{00} f^{000}+3 v_{0}{ }^{1} f^{002}:
\end{aligned}
$$


We remind the reader that $\left.f \circ v_{0}\right)<0$ is already known to yield modulational instability through non hyperbolicity of the dispersionless system. We observe furthermore that in the case under consideration when $\left.\left.f 0 q_{0}\right)>0, f 00 v_{0}\right) \quad 0$ and $\left.f{ }^{000 q} q_{0}\right) \quad 0$ then any $k_{0}$ is admissible and no modulational instability occurs. In particular for the hydrodynamic formulations of cubic Schr odinger equations, that is, when $f{ }^{0}$ is an ane function, modulational instability is completely decided by the sign of $f o q\left(v_{0}\right)$ independently of $k_{0}$, that is, it is driven by the focusing/defocusing nature of the equation.

Going back to the general case (when is arbitrary and is ane), we stress, as in Remark 7 , the consistency of the foregoing computations with the Eulerian/mass Lagrangian conjugation (see [BG13, BGNR14]]). To be more explicit, we denote with subscripts E and $L$ quantities corresponding to each formulation. First we observe that $b_{E}=1$ and $E=I d$, whereas $b_{L}=1$ and $L \quad 0$. Moreover

$$
f_{L}(v)=v f_{E} \frac{1}{v} ; \quad L(v)=\frac{1}{v^{5}} \text { E } \frac{1}{v}
$$

and at the harmonic limit

$$
\left(\mathrm{v}_{\mathrm{L}}\right)_{0}=\frac{1}{\left(\mathrm{v}_{\mathrm{E}}\right)_{0}} ; \quad\left(\mathrm{k}_{\mathrm{L}}\right)_{0}=\frac{\left(\mathrm{k}_{\mathrm{E}}\right)_{0}}{\left(\mathrm{v}_{\mathrm{E}}\right)_{0}}:
$$

Our observation is that when going from mass Lagrangian to Eulerian formulations the third-order polynomial is simply multiplied by $\left(\left(\mathrm{V}_{\mathrm{L}}\right)_{0}\right)^{11}$.

\section{References}

[BG 13] S. Benzoni Gavage. Planar traveling waves in capillary uids. Dierential Integral Equations , 26(3-4):433\{478, 2013.

[BGM R 16] S. B enzoni-Gavage, C. M ietka, and L. M. Rodrigues. Co-periodic stability of periodic waves in some Hamiltonian PDEs. N onlinearity , 29(11):3241, 2016.

[BGMRar] S. Benzoni-Gavage, C. M ietka, and L. M. Rodrigues. Stability of periodic waves in Hamiltonian PDEs of either long wavelength or small amplitude. Indiana Univ. Math. J. , to appear.

[BGNR13] S. Benzoni-Gavage, P. Noble, and L. M. Rodrigues. Stability of periodic waves in Hamiltonian PDEs. In Jourees Equations aux driees partielles , number 2, pages 1\{22. Biarritz, 2013.

[BGNR 14] S. Benzoni-Gavage, P. Noble, and L. M. Rodrigues. Slow modulations of periodic waves in Hamiltonian PDEs, with application to capillary uids. J. Nonlinear Sci. , 24(4):711\{768, 2014. 
[BT 13] M. B ertola and A. Tovbis. Universality for the focusing nonlinear Schr odinger equation at the gradient catastrophe point: rational breathers and poles of the tritronqee solution to Painle I. Comm. Pure A ppl. Math. , 66(5):678\{752, 2013.

[BB 05] S. Bianchini and A. Bressan. Vanishing viscosity solutions of nonlinear hyperbolic systems. Ann. of Math. (2) , 161(1):22\}\{342, 2005.

[Bre00] A. Bressan. Hyperbolic systems of conservation laws, volume 20 of Oxford Lecture Series in Mathematics and its A pplications . Oxford University Press, Oxford, 2000. The one-dimensional Cauchy problem.

[Bri17] T. J. Bridges. Symmetry, phase modulation and nonlinear waves , volume 31 of Cambridge M onographs on A pplied and Computational M athematics . Cambridge University Press, Cambridge, 2017.

[CG 09] T. Claeys and T. Grava. Universality of the break-up prole for the K dV equation in the small dispersion limit using the Riemann-Hilbert approach. Comm. M ath. Phys. , 286(3):979\{1009, 2009.

[CG12] T. Claeys and T. Grava. The K dV hierarchy: universality and a Painlee transcendent. Int. M ath. Res. Not. IM RN ，(22):5063\{5099, 2012.

[Col51] J. D. Cole. On a quasi-linear parabolic equation occurring in aerodynamics. Quart. A ppl. Math. , 9:225\{236, 1951.

[DV Z97] P. Deift, S. Venakides, and X. Zhou. $N$ ew results in small dispersion $\mathrm{K} d V$ by an extension of the steepest descent method for Riemann-Hilbert problems. Internat. M ath. Res. Notices , (6):286\{299, 1997.

[Dub06] B. Dubrovin. On Hamiltonian perturbations of hyperbolic systems of conservation laws. II. Universality of critical behaviour. Comm. Math. Phys. , 267(1):117\{139, 2006.

[DM N76] B. A. Dubrovin, V. B. Matveev, and S. P. Novikov. Nonlinear equations of Korteweg-de $V$ ries type, nite-band linear operators and A belian varieties. U spehi Mat. Nauk, 31(1(187)):55\{136, 1976.

[DN74] B. A. Dubrovin and S. P. Novikov. A periodic problem for the Korteweg-de $\checkmark$ ries and Sturm-Liouville equations. Their connection with al gebraic geometry. Dokl. A kad. Nauk SSSR, 219:531\{534, 1974.

[DN83] B. A . Dubrovin and S. P. Novikov. Hamiltonian formalism of one-dimensional systems of the hydrodynamic type and the Bogolyubov-W hitham averaging method. Dokl. A kad. Nauk SSSR, 270(4):781\{785, 1983. 
[D N89] B. A. Dubrovin and S. P. N ovikov. Hydrodynamics of weakly deformed soliton lattices. Dierential geometry and Hamiltonian theory. Uspekhi Mat. Nauk, $44(6(270)): 29\{98,203,1989$.

[DN93] B. A. Dubrovin and S. P. Novikov. Hydrodynamics of soliton lattices, volume 9 of Soviet Scientic Reviews, Section C: M athematical Physics Reviews Harwood A cademic Publishers G mbH, Y verdon, 1993.

[E105] G. A. El. Resolution of a shock in hyperbolic systems modied by weak dispersion. Chaos, 15(3):037103, 21, 2005.

[EH16] G. A. El and M. A. Hoefer. Dispersive shock waves and modulation theory. Phys. D, 333:11\{65, 2016.

[EK V 01] G. A. El, A. L. K rylov, and S. Venakides. Unied approach to K dV modulations. Comm. Pure A ppl. M ath. , 54(10):1243\{1270, 2001.

[EJ LM 03] N. M. Ercolani, S. Jin, C. D. Levermore, and W. D. MacEvoy, Jr. The zerodispersion limit for the odd ows in the focusing Zakharov-Shabat hierarchy. Int. M ath. Res. Not. , (47):2529\{2564, 2003.

[FFM 80] H. Flaschka, M. G. Forest, and D. W. M CL aughlin. M ultiphase averaging and the inverse spectral solution of the K orteweg-de $V$ ries equation. Comm. Pure A ppl. M ath. , 33(6):739\{784, 1980.

[FL86] M. G. Forest and J. E. Lee. Geometry and modulation theory for the periodic nonlinear Schr odinger equation. In Oscillation theory, computation, and methods of compensated compactness (M inneapolis, M inn., 1985) , volume 2 of IM A Vol. M ath. A ppl., pages 35\{69. Springer, New York, 1986.

[GS95] S. L. Gavrilyuk and D. Serre. A model of a plug-chain system near the thermodynamic critical point: connection with the Korteweg theory of capillarity and modulation equations. In $W$ aves in liquid/gas and liquid/vapour twophase systems (K yoto, 1994), volume 31 of Fluid M ech. A ppl. , pages $419\{428$. K luwer A cad. Publ., Dordrecht, 1995.

[Gra02] T. Grava. Riemann-Hilbert problem for the small dispersion limit of the $\mathrm{K} d V$ equation and linear overdetermined systems of Euler-Poisson-Darboux type. Comm. Pure A ppl. Math. , 55(4):395\{430, 2002.

[Gra04] T. Grava. Whitham equations, Bergmann kernel and Lax-Levermore minimizer. Acta A ppl. Math. , 82(1):1\{86, 2004.

[GT02] T. Grava and F.-R. Tian. The generation, propagation, and extinction of multiphases in the KdV zero-dispersion limit. Comm. Pure Appl. Math. , 55(12):1569\{1639, 2002. 
[GP73] A. V. Gurevich and L. P. Pitaevskii. N onstationary structure of a collisionless shock wave. Zhurnal Eksperimentalnoi i Teoreticheskoi Fiziki , 65:590\{604, 1973.

[Hoe14] M. A. Hoefer. Shock waves in dispersive Eulerian uids. J. Nonlinear Sci. , 24(3):525\{577, 2014.

[Hop50] E. Hopf. The partial dierential equation $u_{t}+u_{x}=u_{x x}$. Comm. Pure Appl. M ath. , 3:201\{230, 1950.

[Jen15] R. Jenkins. Regularization of a sharp shock by the defocusing nonlinear Schrodinger equation. Nonlinearity , 28(7):2131\{2180, 2015.

[JM 14] R. Jenkins and K. D. T.-R. M cLaughlin. Semiclassical limit of focusing NLS for a family of square barrier initial data. Comm. Pure A ppl. Math. , 67(2):246\{ 320, 2014.

[J LM 99] S. Jin, C. D. Levermore, and D. W. M CLaughlin. The semiclassical limit of the defocusing NLS hierarchy. Comm. Pure A ppl. M ath. , 52(5):613\{654, 1999.

[JNRZ13] M. A. Johnson, P. Noble, L. M. Rodrigues, and K. Zumbrun. Nonlocalized modulation of periodic reaction diusion waves: the Whitham equation. Arch. Ration. Mech. Anal. , 207(2):669\{692, 2013.

[J NRZ14] M. A. Johnson, P. Noble, L. M. Rodrigues, and K. Zumbrun. Behavior of periodic solutions of viscous conservation laws under localized and nonlocalized perturbations. Invent. M ath. , 197(1):115\{213, 2014.

[Kam00] A. M. Kamchatnov. Nonlinear periodic waves and their modulations . World Scientic Publishing Co., Inc., River Edge, NJ, 2000. An introductory course.

[LL83] P. D. Lax and C. D. Levermore. The small dispersion limit of the K orteweg-de V ries equation. II. Comm. Pure A ppl. Math. , 36(5):571\{593, 1983.

[M ie17] C. M ietka. Periodic waves in some Hamiltonian PDEs : stability, modulations and dispersive shocks. PhD thesis, U niversid de L yon, 2017.

[M il02] P. D. Miller. A symptotics of semiclassical soliton ensembles: rigorous justication of the WKB approximation. Int. Math. Res. Not. , (8):383\{454, 2002.

[M il16] P. D. Miller. On the generation of dispersive shock waves. Phys. D , 333:66\{83, 2016.

[M W 16] P. D. M iller and A. N. Wetzel. The scattering transform for the B enjamin-O no equation in the small-dispersion limit. Phys. D , 333:185\{199, 2016. 
